\title{
Efficient Synthesis of New 1-[Alkyl(aryl)]-5-(3,3,3-trihalo-2-oxopropylidene)pyrrolidin-2-ones
}

\author{
Alex F. C. Flores, *Darlene C. Flores, Graciela Oliveira, Lucas Pizzuti, Rubia M. S. da Silva, \\ Marcos A. P. Martins and Helio G. Bonacorso
}

\author{
NUQUIMHE - Departamento de Química, Universidade Federal de Santa Maria \\ 97105900 Santa Maria-RS, Brazil
}

\begin{abstract}
Este trabalho mostra a síntese, em bons rendimentos (57-95\%), de duas séries de 1-alquil(aril) amino-2-oxo-5-(2-oxo-3,3,3-trialopropiliden)-pirrolidinas 5 e $\mathbf{6}$, a partir dos intermediários 4-[alquil(aril)amino]-6-oxo-7,7,7-trialo-4-hepteno-atos de metila $\mathbf{3}$ e $\mathbf{4}$ obtidos por substituição da metoxila- $\beta$ nos precursores 4-metoxi-6-oxo-7,7,7-trialo-4-heptenoatos de metila 1 e 2 , pela série de aminas primárias $\mathrm{RNH}_{2}$, onde $\mathrm{R}=\mathrm{PhCH}_{2}, \mathrm{PhCH}_{2} \mathrm{CH}_{2}, \mathrm{Ph}, 4-\mathrm{MeC}_{6} \mathrm{H}_{4}, 4-\mathrm{MeOC}_{6} \mathrm{H}_{4}$, 4- $\mathrm{ClC}_{6} \mathrm{H}_{4}, 4-\mathrm{BrC}_{6} \mathrm{H}_{4}, 2$-piridnil, 5-metil-3-isoxazolil, 4- $\mathrm{NH}_{2} \mathrm{C}_{6} \mathrm{H}_{4}$. A estrutura molecular dos produtos inéditos foi atribuída a partir dos dados de $\mathrm{RMN}{ }^{1} \mathrm{H},{ }^{13} \mathrm{C}$ e espectrometria de massas. A configuração geométrica dos compostos $\mathbf{3 d}$ e $\mathbf{5 b}$ foi confirmada pelos dados de difração de raios-X em monocristal.
\end{abstract}

Reactions of methyl 4-methoxy-6-oxo-7,7,7-trihalo-4-heptenoates $\mathbf{1}$ and $\mathbf{2}$ with primary amines $\mathrm{RNH}_{2}$, where $\mathrm{R}=\mathrm{PhCH}_{2}, \mathrm{PhCH}_{2} \mathrm{CH}_{2}, \mathrm{Ph}, 4-\mathrm{MeC}_{6} \mathrm{H}_{4}, 4-\mathrm{MeOC}_{6} \mathrm{H}_{4}, 4-\mathrm{ClC}_{6} \mathrm{H}_{4}, 4-\mathrm{BrC}_{6} \mathrm{H}_{4}$, 2-pyridyl, 5-methyl-3-isoxazolyl, 4- $\mathrm{NH}_{2} \mathrm{C}_{6} \mathrm{H}_{4}$ affording methyl 4-[alkyl(aryl)amino]-6-oxo-7,7,7trihalo-4-heptenoates 3, 4, in good yields (57-95\%), which suffer quantitative intramolecular cyclocondensation to produce 1-alkyl(aryl)-5-(2-oxo-3,3,3-trihalopropylidene)pyrrolidin-2-ones $\mathbf{5 , 6}$, are reported. The structures of the isolated new products were assigned by means of ${ }^{1} \mathrm{H},{ }^{13} \mathrm{C}$ NMR measurements and mass spectrometry. The $Z$ and $E$ configuration of compounds $\mathbf{3 d}$ and $\mathbf{5 b}$ respectively were established from X-ray crystallography.

Keywords: pyrrolidin-2-ones, [CCCC+N] cyclisation, methyl 4-methoxy-6-oxo-7,7,7-trihalo4-heptenoates

\section{Introduction}

We have systematically used the acetals acylation method for synthesis of wide range of 4-alkoxy-1,1,1-trihalo-3-alken2-ones. These 1,3-dielectrophilic precursors have proved to be important building blocks for regiospecific synthesis of heterocyclic compounds bearing trihalomethyl group ${ }^{2,3}$ with important pharmacological ${ }^{4,5}$ and synthetic applications. ${ }^{6}$ The levulinic acid (4-oxopentanoic acid) is an important fine organic material from renewable source, with acetyl group attractive for us. ${ }^{7}$ Our continuing interest in 1,3-dielectrophilic compounds has led us to study a new aspect of the application of the acetal acylation method for producing methyl 4-methoxy-6-oxo-7,7,7-trihalo-4-heptenoates 1 and 2. ${ }^{8}$

On the other hand, the pyrrolidin-2-ones, have received considerable attention due to their activity in $\mathrm{CNS}$ as

*e-mail: alexflores@smail.ufsm.br nootropic drugs. Piracetam-like nootropics revert amnesia induced by scopolamine and other amnesing drugs, electroconvulsive shock and hypoxia with an unknown mechanism. ${ }^{9}$ In general, they show no affinity for the most important central receptors, but are able to modulate the action of these most important central neurotransmitters, in particular acetylcholine and glutamate. Extensive study of the modes of action of the 2-pyrrolidinones has revealed various pharmacological effects, with striking differences between drugs. ${ }^{10,11}$

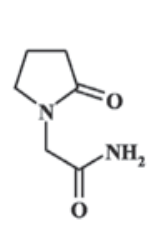

Piracetam
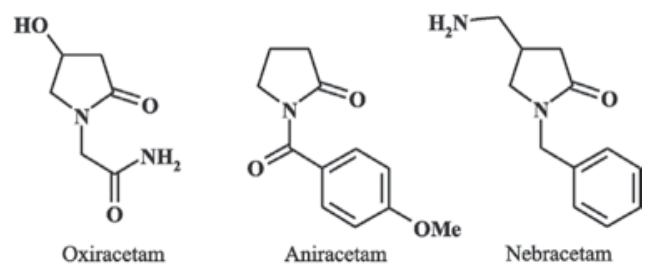

Figure 1. Structures of pyrrolidin-2-one drugs. 
Continuing with our systematic studies on synthesis and application of 4-alkoxy-1,1,1-trihalo-3-alken-2-ones we report an efficient approach for synthesis of a series of 1-[alkyl(aryl)]-5-(3,3,3-trihalo-2-oxopropylidene)pyrrolidin-2-nes from $\mathbf{1}$ and $\mathbf{2}$. This study deals with formation of the methyl 4-alkyl[aryl]amino-6-oxo-7,7,7-trihalo-4heptenoates $\mathbf{3}$ and $\mathbf{4}$, and their intramolecular cyclisations to new 1-alkyl[aryl]-5-(3,3,3-trihalo-2-oxopropylidene) pyrrolidin-2-ones $\mathbf{5}$ and $\mathbf{6}$.

\section{Results and Discussion}

The precursors methyl 4-methoxy-6-oxo-7,7,7-trihalo4-heptenoates $\mathbf{1}$ and $\mathbf{2}$ were obtained by acetal acylation method as described previously. ${ }^{8}$

The fluorinated enaminones $\mathbf{3}$ were synthesized and isolated by reaction of the methyl 4-methoxy-6-oxo-7,7,7trifluoro-4-heptenoate (1) with primary alkyl[aryl]amines in $\mathrm{CH}_{3} \mathrm{CN}$ at variable temperatures depending on used amine. The analytical data have demonstrated the formation of the methyl 4-alkyl[aryl]amino-6-oxo-7,7,7-trifluoro-4heptenoates 3,b-k (Table 1, Supplementary Information), with (Z)-4-alkyl[aryl]amino-1,1,1-trifluoro-3-alken-2-one moiety. ${ }^{2}$ Primary alkylamines $(\mathbf{b}, \mathbf{c})$ reacted readily with 4-methoxy-1,1,1-trifluoro-3-alken-2-ones in $\mathrm{MeOH}, \mathrm{EtOH}$ or $i$-PrOH by displacement of $\beta$-methoxy substituent, in these cases, the intramolecular cyclocondensation of the resulting methyl 4-alkylamino-6-oxo-7,7,7-trifluoro-4heptenoates 3,b,c to pyrrolidin-2-ones also took place, affording mixtures of products $\mathbf{3}$ and $\mathbf{5}$ at room temperature. However, when the least reactive primary arylamines were employed, and the reaction was carried out in $\mathrm{MeOH}$ or $\mathrm{EtOH}$ at room temperature, unreacted 1 or mixtures of methyl 4-ethoxy-6-oxo-7,7,7-trifluoro-4-heptenoate and $\mathbf{1}$ were recovered. The methyl 4-arylamino-6-oxo-7,7,7-trifluoro4-heptenoates 3,d-j were obtained in refluxing $\mathrm{MeCN}$ or at room temperature, by addition of equimolar amount of $\mathrm{Et}_{3} \mathrm{~N}$ or lutidine (Table 1, Supplementary Information). Reaction of the methyl 4-methoxy-6-oxo-7,7,7-trifluoro-4-heptenoate 1 with 1,4-diaminobenzene in a stoichiometric ratio 1:1 or in 2:1 mol-equiv ratio in $\mathrm{MeCN}$ at room temperature lead to the methyl 4-(4-aminophenylamino)-6-oxo-7,7,7trifluoro-4-heptenoate $\mathbf{3 k}$, but under reflux conditions the intramolecular cyclisation took place under formation of only 1,1'-(1,4-phenylene)-bis(5-(3,3,3-trifluoro-2oxopropylidene)-2-pyrrolidin-2-one) $\mathbf{5 k}$ (Scheme 1).

The structures of fluorinated enaminones $\mathbf{3}$ were confirmed by elemental analysis, mass spectrometry and ${ }^{1} \mathrm{H}$, ${ }^{13} \mathrm{C}$ NMR spectral data. The ${ }^{1} \mathrm{H}$ NMR spectra showed characteristic broad signals from $\mathrm{N}-\mathrm{H}$ between 12.4-13.2 ppm, the vinylic hydrogen singlet at 5.47-5.68 ppm

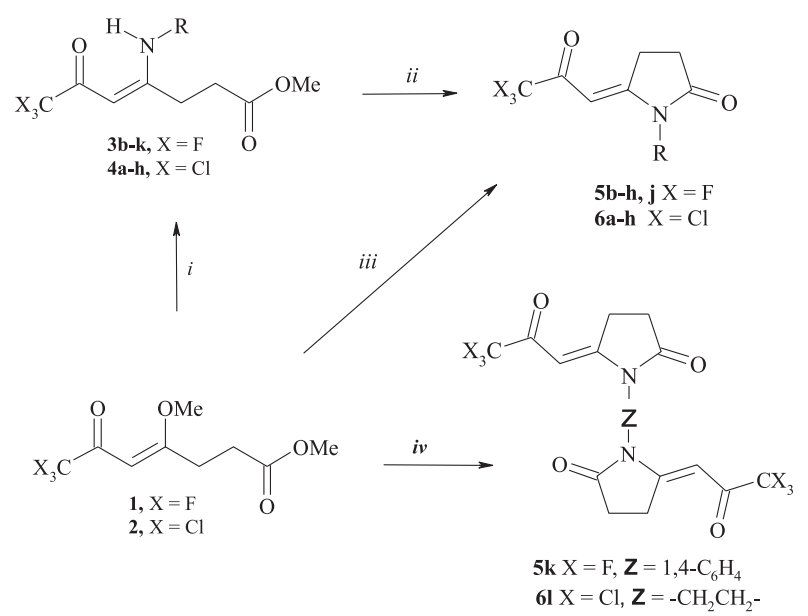

$$
\begin{aligned}
& \begin{array}{c|ccccccc} 
& \mathrm{a} & \mathrm{b} & \mathrm{c} & \mathrm{d} & \mathrm{e} & \mathrm{f} & \mathrm{g} \\
\hline \mathrm{R} & \mathrm{n}-\mathrm{Pr} & \mathrm{CH}_{2} \mathrm{Ph} & \left(\mathrm{CH}_{2}\right)_{2} \mathrm{Ph} & \mathrm{Ph} & 4-\mathrm{Me}-\mathrm{C}_{6} \mathrm{H}_{4} & 4-\mathrm{MeO}-\mathrm{C}_{6} \mathrm{H}_{4} & 4-\mathrm{Cl}-\mathrm{C}_{6} \mathrm{H}_{4}
\end{array}
\end{aligned}
$$

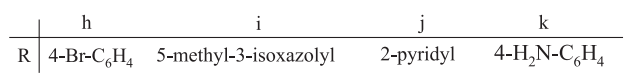

$$
\begin{aligned}
& \text { i. } \mathrm{RNH}_{2}, \mathrm{MeCN}, 25-80^{\circ} \mathrm{C}, 6-24 \mathrm{~h} \text {. ii. } \mathrm{Et}_{3} \mathrm{~N}, \mathrm{MeCN}, 80^{\circ} \mathrm{C}, 24 \mathrm{~h}, 75-95 \% \text {. } \\
& \text { iii. (a) } \mathrm{RNH}_{2}, \mathrm{MeCN}, 25-80^{\circ} \mathrm{C}, 12 \mathrm{~h} \text {; (b) } \mathrm{Et}_{3} \mathrm{~N}, 80^{\circ} \mathrm{C}, 8-24 \mathrm{~h} \\
& \text { iv. 1,4-diaminobenzene or ethylenediamine, } \mathrm{MeCN}, 25-80^{\circ} \mathrm{C}, 12 \mathrm{~h} \text {. }
\end{aligned}
$$

Scheme 1.

and for methyl group of ester as intense singlet at 3.64-3.70 ppm. ${ }^{12,13}$ The ${ }^{13} \mathrm{C}$ NMR spectra showed the signals of methoxy group at 51.5-51.9 ppm and carboxylic ester carbon at $169.8-170.7 \mathrm{ppm}$ confirming the propionyl chain, two characteristic quartets for carbonyl carbon C6, ca. 176-178 ppm, ${ }^{2} J_{\mathrm{CF}} 34 \mathrm{~Hz}$ and trifluoromethyl group C7, ca. $116.5-117.5 \mathrm{ppm},{ }^{1} J_{\mathrm{CF}} 288 \mathrm{~Hz}$, all confirming acyclic enaminone structures 3b-k .

Reacting 1 or 2 with 5-amino-3-methyl-1H-pyrazole under conditions described above, the products of cyclocondensation [3 + 3], the methyl 3-(2-methyl-7-(trihalomethyl) pyrazolo[1,5-a]pyrimidin-5-yl)propanoates $\mathbf{7}$ and $\mathbf{8}$ (Scheme 2). Their structures were confirmed by elemental analysis and ${ }^{1} \mathrm{H},{ }^{13} \mathrm{C} \mathrm{NMR}$ data. ${ }^{15}$

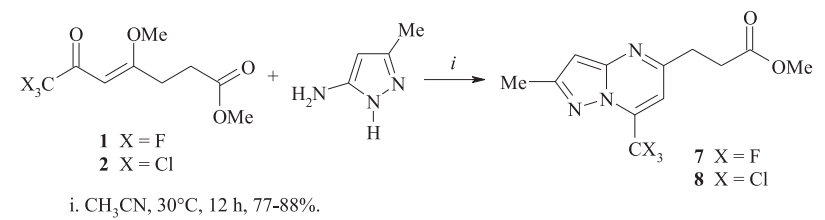

Scheme 2. 5-amino-3-methyl-1H-pyrazole lead to $[\mathrm{CCC}+\mathrm{NCN}]$ cyclisation.

The easy intramolecular cyclization of methyl 4-alkylamino-6-oxo-7,7,7-trifluoro-4-heptenoates 3b,c to pyrrolidin-2-ones $\mathbf{5 b}$,c occurred in $\mathrm{MeOH}$, EtOH or MeCN. The intramolecular cyclisation of methyl 4-alkylamino-6oxo-7,7,7-trifluoro-4-heptenoates 3d-j to 5-(3,3,3-trifluoro2-oxopropylidene)-pyrrolidin-2-ones $\mathbf{5 d - j}$ occurred only 
in refluxing $\mathrm{MeCN}$ in the presence of equimolar amount of $\mathrm{Et}_{3} \mathrm{~N}$ for reaction periods varying between 8-24 h. The products $\mathbf{5 , b - k}$ can be obtained in one pot process by the reaction of precursor 1 and amine in $\mathrm{MeCN}$ followed by $\mathrm{Et}_{3} \mathrm{~N}$ addition and/or reflux $\left(85^{\circ} \mathrm{C}\right)$ affording similar yields.

The 1-Alkyl(aryl)-5-(3,3,3-trifluoro-2-oxopropylidene) pyrrolidin-2-ones $\mathbf{5 , b - k}$ were characterised by elemental analysis, mass spectrometry and ${ }^{1} \mathrm{H},{ }^{13} \mathrm{C}$ NMR data. The ${ }^{1} \mathrm{H}$ NMR spectra showed characteristic signals for vinylic hydrogen at 5.66-5.94 ppm and the signals for cyclic $\mathrm{CH}_{2}$ as multiplet at 2.67-2.83 and 3.33-3.57 ppm. The ${ }^{13} \mathrm{C}$ NMR spectra showed the signals of amide carbon at 167.8-169.2 ppm, two characteristic quartets of carbonyl carbon at propylidene chain $\mathrm{ca}$. 178-179.3 ppm, ${ }^{2} J_{\mathrm{CF}} 34 \mathrm{~Hz}$, for trifluoromethyl group $\mathrm{CF}_{3} c a$. 116.2-116.4 ppm, ${ }^{1} J_{\mathrm{CF}}$ $291 \mathrm{~Hz}$ and for vinylic carbon at 90.3-93.7 ppm, all confirming 1-alkyl[aryl]-5-(3,3,3-trifluoro-2-oxopropylidene) pyrrolidin-2-one structures $\mathbf{5 , b - k}$.

Compounds $\mathbf{3 d}$ and $\mathbf{5 b}$ were subjected to single crystal X-ray analysis (Tables 4 and 5). This measurement has showed in solid state the molecular structure with $Z$-configuration on alkene moiety and intramolecular hydrogen bond O3---H1-N1 for methyl 6-oxo-4-phenylamino-7,7,7-trifluoro-4-heptenoate (3d) (Figure 2). $\mathrm{X}$-ray diffraction analysis performed on 1-benzyl-5-(3,3,3trifluoro-2-oxopropylidene)pyrrolidin-2-one (5b) showed the $E$-configuration for enaminoketone moiety (Figure 3), confirming that the intramolecular cyclisation occurred with the inversion of configuration. In both structures the trifluoromethyl group suffers a rotational disorder and was refined as two components as approximately $60 \% / 40 \%$ occupancy (Tables 1 and 2).

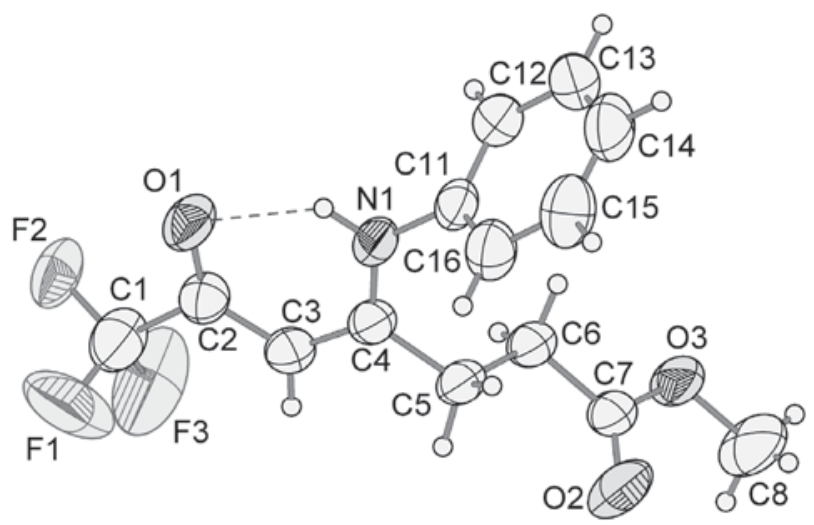

Figure 2. ORTEP representation of the X-ray molecular structure of methyl 4-benzylamino-2-oxo-7,7,7-trifluoro-4-heptanoate (3d).

The methyl 4-methoxy-6-oxo-7,7,7-trichloro-4heptenoate demonstrated high reactivity for nucleophilic attack of alkylamines at $\beta$-carbon of the vinyl group, taking place of methoxy group, followed by fast intramolecular

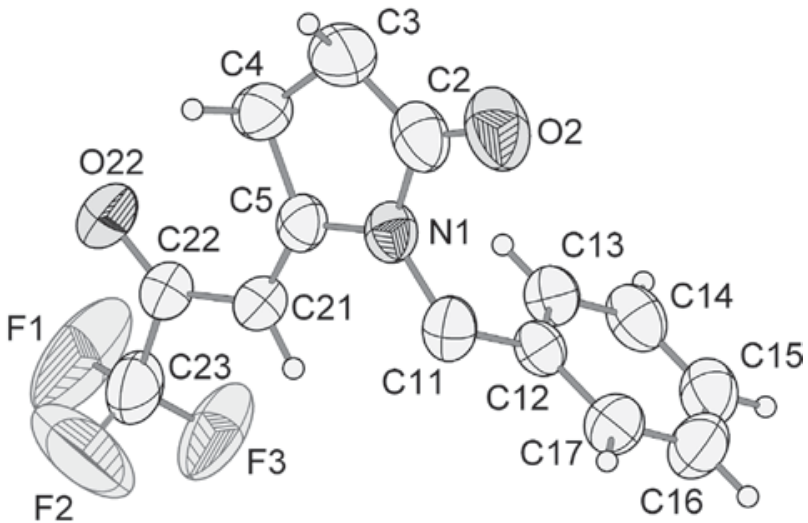

Figure 3. ORTEP representation of the X-ray molecular structure of 1-benzyl-5-(3,3,3-trifluoro-2-oxopropylidene)pyrrolidin-2-one (5b).

cyclisation to 1-alkyl-5-(3,3,3-trichloro-2-oxopropylidene)pyrrolidin-2-ones. With the intention to isolate of the methyl 4-alkylamino-6-oxo-7,7,7-trichloro-4-heptenoates 4,a,c, the heptanoate precursor 2 was reacted with primary alkylamines in dichloromethane or chloroform at $-5-0{ }^{\circ} \mathrm{C}$ for 2 hours. Primary arylamines were reacted in $\mathrm{MeCN}$ at room temperature (Scheme 1) for 6 hours, and 4-arylamino6-oxo-7,7,7-trichloro-4-heptenoates $\mathbf{4 , d - j}$ were isolated in good yields and in high purity (Table 2, Supplementary Information). Reactions in $\mathrm{EtOH}$ or $i$-PrOH gave poor yields with complex mixtures of unidentified products, maybe involving transesterication and/or chloroform elimination.

The molecular structures of methyl 4-alkyl[aryl]amino6-oxo-7,7,7-trichloro-4-heptenoates 4,a,c-j were confirmed by elemental analysis, mass spectrometry and ${ }^{1} \mathrm{H},{ }^{13} \mathrm{C}$ NMR spectral data. The ${ }^{1} \mathrm{H}$ NMR spectra showed characteristic broad signals for N-H between 13.4-13.8 ppm, the vinylic hydrogen at 5.85-5.89 ppm and for methoxy group as intense singlet at 3.60-3.67 ppm. ${ }^{12,13}$ The ${ }^{13} \mathrm{C}$ NMR spectra showed the signals of methoxy group at characteristic region 51.9-52.9 ppm, for the carboxylic ester carbon at 181.2-181.7 ppm and low intense signal at 96-99.1 for trichloromethyl carbon confirming the attributed structure for methyl 4-alkyl[aryl]amino-6-oxo-7,7,7-trichloro-4heptenoates $\mathbf{4 , a , c - j . ~}$

The intramolecular cyclisation of isolated methyl 4-alkyl[aryl]amino-6-oxo-7,7,7-trichloro-4-heptenoates 4,a,c to 5-(3,3,3-trichloro-2-oxopropylidene)pyrrolidin2-ones 6,a,c occurred in $\mathrm{MeCN}$, affording quantitative yields without $\mathrm{Et}_{3} \mathrm{~N}$ addition. The products $\mathbf{6 , d - f}$ and $\mathbf{6 h}$ were obtained in good yields by stirring of $\mathbf{4}$ in $\mathrm{MeCN}$ at variable temperatures for 2 to $6 \mathrm{~h}$. In general, the products 6 can be readily obtained in excellent yields without isolation of enaminones intermediates, by the reaction of precursor $\mathbf{2}$ and appropriate amine in $\mathrm{MeCN}$ under reflux 
temperature $\left(80-85^{\circ} \mathrm{C}\right)$. The $1,1^{\prime}$-(ethane-1,2-diyl)bis $(5$ (3,3,3-trichloro-2-oxopropylidene)pyrrolidin-2-one) $6 \mathbf{l}$ was obtained by the reaction of 2 and ethylenediamine in 2:1 equiv ratio in $\mathrm{MeCN}$ at room temperature (Scheme 1).

The 1-alkyl[aryl]-5-(3,3,3-trichloro-2-oxopropylidene) pyrrolidin-2-ones $\mathbf{6 a}, \mathbf{6 c - f}$ and $\mathbf{6 h}$ were characterised by elemental analysis, mass spectrometry and ${ }^{1} \mathrm{H},{ }^{13} \mathrm{C}$ NMR data. 2D COSY ( $\mathrm{HH}$ and $\mathrm{CH})$ and DEPT experiments were used for signal assignment.

Table 1. Crystal data and structure refinement for $\mathbf{3 d}$ and $\mathbf{5 b}$

\begin{tabular}{|c|c|c|}
\hline Crystal data & 3d & $5 b$ \\
\hline CCDC No.a & 615368 & 615367 \\
\hline Formula & C14H14F3NO3 & $\mathrm{C} 14 \mathrm{H} 12 \mathrm{~F} 3 \mathrm{NO} 2$ \\
\hline Habit & Colorless & Colorless \\
\hline Size $(\mathrm{mm})$ & $0.15 \times 0.15 \times 0.1$ & $0.35 \times 0.15 \times 0.13$ \\
\hline Symmetry & Monoclinic, P2(1)/c & $\begin{array}{l}\text { Orthorhombic, } \\
\text { Pbca }\end{array}$ \\
\hline \multicolumn{3}{|l|}{ Unit cell dimensions $(\AA)$} \\
\hline $\mathrm{a}, \alpha$ & $11.2299(5), 90^{\circ}$ & $9.6444(7), 90^{\circ}$ \\
\hline$b, \beta$ & $5.9503(2), 104.22^{\circ}$ & $15.5012(7), 90^{\circ}$ \\
\hline $\mathrm{c}, \gamma$ & $22.8256(10), 90^{\circ}$ & $17.7423(9), 90^{\circ}$ \\
\hline Volume (A3), Z & 1478.46(10), 4 & $2652.5(3), 8$ \\
\hline Dc (g.cm-3) & 1.353 & 1.419 \\
\hline$\mu(\mathrm{mm}-1)$ & 0.119 & 0.123 \\
\hline $\begin{array}{l}\theta \text { range for data col- } \\
\text { lection }\left(^{\circ}\right)\end{array}$ & $3.55<\theta<30.09$ & $2.74<\theta<27.0$ \\
\hline Index ranges & $\begin{aligned}-15 & \leq \mathrm{h} \leq 15 \\
-8 & \leq \mathrm{k} \leq 8 \\
-31 & \leq 1 \leq 29\end{aligned}$ & $\begin{array}{l}-5 \leq \mathrm{h} \leq 12 \\
-19 \leq \mathrm{k} \leq 18 \\
-22 \leq 1 \leq 22\end{array}$ \\
\hline Reflexions collected & 17625 & 14247 \\
\hline $\begin{array}{l}\text { Independent reflexions } \\
\text { (Rint) }\end{array}$ & $\begin{array}{c}4275 \\
(0.0313)\end{array}$ & $\begin{array}{c}2895 \\
0.0396\end{array}$ \\
\hline Completeness to $\theta$ & $98.4 \%$ & $99.8 \%$ \\
\hline Tmin- Tmax & $0.864-1.0$ & $0.6937-1.0$ \\
\hline Solution & \multicolumn{2}{|c|}{ Direct methods SHELXS-97 } \\
\hline Refinement method & \multicolumn{2}{|c|}{ Full-matrix least-squares on F2 } \\
\hline $\begin{array}{l}\text { Data, restraints, } \\
\text { parameters }\end{array}$ & $4275,48,228$ & $2895,0,195$ \\
\hline Goodness-of-fit on F2 & 1.043 & 1.072 \\
\hline $\begin{array}{l}\text { Final } R \text { indices }[I> \\
2 \sigma(I)]\end{array}$ & $\begin{array}{c}\mathrm{R} 1=0.0494 \\
\mathrm{wR} 2=0.1343\end{array}$ & $\begin{array}{c}\mathrm{R} 1=0.0662 \\
\mathrm{wR} 2=0.1951\end{array}$ \\
\hline R índices (all data) & $\begin{array}{c}\mathrm{R} 1=0.0975 \\
w R 2=0.1576\end{array}$ & $\begin{array}{c}\mathrm{R} 1=0.1047 \\
\mathrm{wR} 2=0.2254\end{array}$ \\
\hline $\begin{array}{l}\text { Largest diff. peak } \\
\text { and hole (e. } \AA \text {-3) }\end{array}$ & 0.23 and -0.227 & 0.337 and -0.535 \\
\hline
\end{tabular}

a CCDC 286548 contains the supplementary crystallographic data for this paper. These data can be made available free of charge via www. ccdc.cam.ac.uk/data_request/cif, by emailing data_request@ccdc.cam. ac.uk or by contacting CCDC.
Table 2. Bond lengths $[\AA]$ and angles $\left[{ }^{\circ}\right]$ for $\mathbf{3 d}$ and $\mathbf{5 b}$

\begin{tabular}{|c|c|c|c|}
\hline \multicolumn{2}{|c|}{$3 d$} & \multicolumn{2}{|c|}{$5 b$} \\
\hline C7-F1 & $1.3215(10)$ & $\mathrm{N} 1-\mathrm{C} 2$ & $1.398(4)$ \\
\hline C7-F2 & $1.3219(10)$ & N1-C5 & $1.369(3)$ \\
\hline C7-F3 & $1.3224(10)$ & N1-C11 & $1.454(3)$ \\
\hline C7-C6 & $1.528(2)$ & $\mathrm{C} 2-\mathrm{O} 2$ & $1.209(4)$ \\
\hline N1-C4 & $1.3339(19)$ & $\mathrm{C} 2-\mathrm{C} 3$ & $1.488(5)$ \\
\hline N1-C11 & $1.4287(19)$ & $\mathrm{C} 3-\mathrm{C} 4$ & $1.517(5)$ \\
\hline N1-H1 & 0.86 & C3-H3A & 0.97 \\
\hline C6-O3 & $1.2350(18)$ & C3-H3B & 0.97 \\
\hline C6-C5 & $1.400(2)$ & C4-C5 & $1.503(4)$ \\
\hline $\mathrm{C} 1-\mathrm{O} 1$ & $1.190(2)$ & C4-H4A & 0.97 \\
\hline $\mathrm{C} 5-\mathrm{C} 4$ & $1.378(2)$ & C4-H4B & 0.97 \\
\hline $\mathrm{C} 5-\mathrm{H} 5$ & 0.93 & $\mathrm{C} 5-\mathrm{C} 21$ & $1.356(4)$ \\
\hline $\mathrm{C} 4-\mathrm{C} 3$ & $1.505(2)$ & $\mathrm{C} 21-\mathrm{C} 22$ & $1.432(4)$ \\
\hline $\mathrm{C} 3-\mathrm{C} 2$ & $1.510(2)$ & $\mathrm{C} 21-\mathrm{H} 21$ & 0.93 \\
\hline C3-H3A & 0.97 & $\mathrm{C} 22-\mathrm{O} 22$ & $1.214(3)$ \\
\hline C3-H3B & 0.97 & $\mathrm{C} 22-\mathrm{C} 23$ & $1.566(4)$ \\
\hline $\mathrm{C} 2-\mathrm{C} 1$ & $1.499(2)$ & $\mathrm{C} 11-\mathrm{C} 12$ & $1.505(4)$ \\
\hline $\mathrm{C} 2-\mathrm{H} 2 \mathrm{~A}$ & 0.97 & C11-H11A & 0.97 \\
\hline $\mathrm{C} 2-\mathrm{H} 2 \mathrm{~B}$ & 0.97 & C11-H11B & 0.97 \\
\hline C1-O1 & $1.322(5)$ & C12-C13 & $1.372(4)$ \\
\hline $\mathrm{C} 1-\mathrm{O} 2$ & $1.33(2)$ & $\mathrm{C} 12-\mathrm{C} 17$ & $1.386(4)$ \\
\hline $\mathrm{O} 2-\mathrm{C} 8$ & $1.434(5)$ & $\mathrm{C} 13-\mathrm{C} 14$ & $1.386(4)$ \\
\hline C8-H8A & 0.96 & C13-H13 & 0.93 \\
\hline C8-H8B & 0.96 & C14-C15 & $1.365(5)$ \\
\hline $\mathrm{C} 8-\mathrm{H} 8 \mathrm{C}$ & 0.96 & C14-H14 & 0.93 \\
\hline $\mathrm{C} 11-\mathrm{C} 16$ & $1.378(2)$ & $\mathrm{C} 15-\mathrm{C} 16$ & $1.366(5)$ \\
\hline $\mathrm{C} 11-\mathrm{C} 12$ & $1.380(2)$ & C15-H15 & 0.93 \\
\hline $\mathrm{C} 12-\mathrm{C} 13$ & $1.379(2)$ & $\mathrm{C} 16-\mathrm{C} 17$ & $1.379(5)$ \\
\hline $\mathrm{C} 12-\mathrm{H} 12$ & 0.93 & C16-H16 & 0.93 \\
\hline $\mathrm{C} 13-\mathrm{C} 14$ & $1.376(3)$ & C17-H17 & 0.93 \\
\hline $\mathrm{C} 13-\mathrm{H} 13$ & 0.93 & $\mathrm{C} 23-\mathrm{F} 1$ & $1.3207(10)$ \\
\hline C14-C15 & $1.374(3)$ & $\mathrm{C} 23-\mathrm{F} 2$ & $1.3210(10)$ \\
\hline $\mathrm{C} 15-\mathrm{C} 16$ & $1.378(3)$ & C23-F3 & $1.3215(10)$ \\
\hline $\mathrm{F} 1-\mathrm{C} 7-\mathrm{F} 2$ & $106.95(16)$ & $\mathrm{C} 2-\mathrm{N} 1-\mathrm{C} 5$ & $112.6(2)$ \\
\hline F1-C7-C6 & $115.2(4)$ & C5-N1-C11 & $125.9(2)$ \\
\hline C4-N1-C11 & $126.6(13)$ & C2-N1-C11 & $121.5(2)$ \\
\hline C4-N1-H1 & 116.7 & $\mathrm{O} 2-\mathrm{C} 2-\mathrm{N} 1$ & $122.3(3)$ \\
\hline C11-N1-H1 & 116.7 & $\mathrm{O} 2-\mathrm{C} 2-\mathrm{C} 3$ & $129.4(3)$ \\
\hline $\mathrm{O} 3-\mathrm{C} 6-\mathrm{C} 5$ & 127.01(15) & $\mathrm{N} 1-\mathrm{C} 2-\mathrm{C} 3$ & $108.3(3)$ \\
\hline O3-C6-C7 & $115.67(14)$ & $\mathrm{C} 2-\mathrm{C} 3-\mathrm{C} 4$ & $105.4(3)$ \\
\hline $\mathrm{C} 5-\mathrm{C} 6-\mathrm{C} 7$ & $117.26(13)$ & $\mathrm{C} 2-\mathrm{C} 3-\mathrm{H} 3 \mathrm{~A}$ & 110.7 \\
\hline C4-C5-C6 & $123.25(14)$ & $\mathrm{C} 4-\mathrm{C} 3-\mathrm{H} 3 \mathrm{~A}$ & 110.7 \\
\hline C4-C5-H5 & 118.4 & H3A-C3-H3B & 108.8 \\
\hline C6-C5-H5 & 118.4 & $\mathrm{C} 5-\mathrm{C} 4-\mathrm{C} 3$ & $105.0(2)$ \\
\hline
\end{tabular}


Table 2. continuation

\begin{tabular}{cccc}
\hline & 3d & & 5 \\
\hline N1-C4-C5 & $121.74(14)$ & C5-C4-H4A & 110.8 \\
N1-C4-C3 & $119.16(14)$ & C3-C4-H4A & 110,8 \\
C5-C4-C3 & $119.08(13)$ & H4A-C4-H4B & 108.8 \\
C4-C3-C2 & $113.15(13)$ & C21-C5-N1 & $123.8(2)$ \\
C4-C3-H3A & 108.9 & C21-C5-C4 & $127.5(2)$ \\
C2-C3-H3A & 108.9 & N1-C5-C4 & $108.7(2)$ \\
H3A-C3-H3B & 107.8 & N1-C11-C12 & $115.0(2)$ \\
C1-C2-C3 & $111.48(13)$ & N1-C11-H11A & 108.5 \\
O1-C1-O2 & $122.2(3)$ & C12-C11-H11A & 108.5 \\
O1-C1-C2 & $124.75(15)$ & C12-C11-H11B & 108.5 \\
O2-C1-C2 & $113.0(3)$ & C13-C12-C17 & $118.5(3)$ \\
C1-O2-C8 & $116.9(4)$ & C13-C12-C11 & $123.0(2)$ \\
C16-C11-C12 & $120.13(16)$ & C17-C12-C11 & $118.5(2)$ \\
C16-C11-N1 & $121.01(15)$ & C12-C13-C14 & $120.6(3)$ \\
C12-C11-N1 & $118.83(15)$ & C12-C13-H13 & 119.7 \\
C13-C12-C11 & $120.06(18)$ & C14-C13-H13 & 119.7 \\
C11-C12-H12 & 120.0 & C15-C14-C13 & $120.2(3)$ \\
C14-C13-C12 & $119.83(19)$ & C14-C15-C16 & $120.0(3)$ \\
C15-C14-C13 & $119.93(19)$ & C15-C16-C17 & $120.0(3)$ \\
C14-C15-C16 & $120.6(2)$ & C16-C17-C12 & $120.7(3)$ \\
C11-C16-C15 & $119.44(19)$ & C5-C21-C22 & $122.3(2)$ \\
C13-C12-H12 & 120.0 & O22-C22-C21 & $128.4(3)$ \\
\hline & 120.1 & O22-C22-C23 & $115.7(2)$ \\
C13-H13 & 119.7 & C21-C22-C23 & $116.0(2)$ \\
\hline
\end{tabular}

The synthesis of 5-(3,3,3-trifluoro-2-oxopropylidene) pyrrolidin-2-ones from methyl 7,7,7-trihalo-4-methoxy6-oxo-4-heptenoates and primary amines can be characterised as an Paal-Knorr process for pyrrole heterocyclic from $[\mathrm{CCCC}+\mathrm{N}]$ building blocks. Mechanistically, it is conceivable that the reaction involves an initial attack from amines to $\beta$-position, leading to enaminoketone moiety, ${ }^{14}$ then an imino intermediate as shown in Scheme 3, which allow the rotation of trihalocarbonyl group around C4-C5 bond on 4-amino-6-oxo-7,7,7-trihalo-4-heptenoates, and intramolecular nucleophilic attack of imino nitrogen to ester carbonyl lead to pyrrolidin-2-ones.

\section{Experimental}

\section{General}

$\mathrm{EtOH}, \mathrm{MeOH}, \mathrm{MeCN}$ and $\mathrm{Et}_{3} \mathrm{~N}$ were purchased from Merck and Aldrich, $\mathrm{R}^{1} \mathrm{NH}_{2}$ was purchased from Aldrich, all used as obtained from suppliers. The methyl 7,7,7-trifluoro

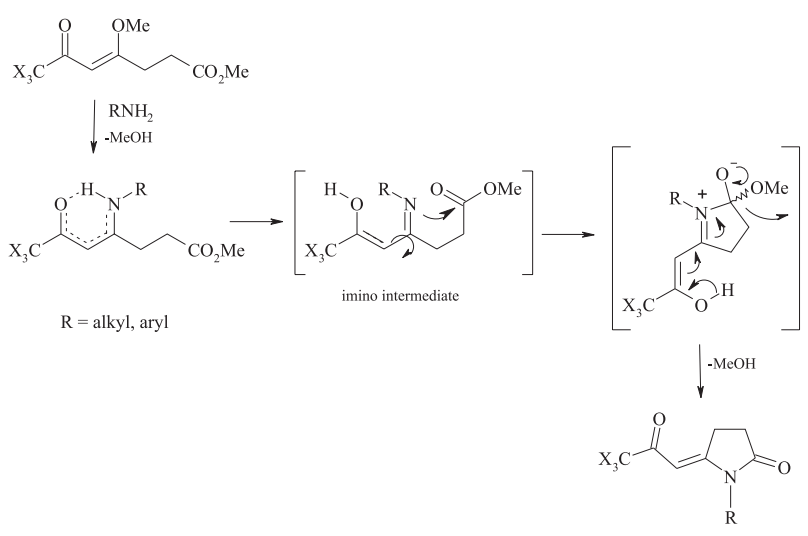

Scheme 3.

[chloro]-4-methoxy-6-oxo-4-heptenoates $\mathbf{1}$ and $\mathbf{2}$ were prepared according to reference 8 . All melting points were determined on a Reichert Thermovar instrument and are uncorrected. ${ }^{1} \mathrm{H}$ and ${ }^{13} \mathrm{C}$ NMR spectra were obtained on a Bruker DPX 400 spectrometer $\left({ }^{1} \mathrm{H}\right.$ at $400.13 \mathrm{MHz}$ and ${ }^{13} \mathrm{C}$ at $100.62 \mathrm{MHz}) 5 \mathrm{~mm}$ sample tubes, $298 \mathrm{~K}$, digital resolution $\pm 0.01 \mathrm{ppm}$, in $\mathrm{CDCl}_{3}$ 0.02-0.05 g. $\mathrm{mL}^{-1}$, using TMS as internal standard. Mass spectra were registered with a HP 6890 GC connected to a HP 5973 MSD and interfaced by a Pentium PC. The GC was equipped with a capillary column ( $30 \mathrm{~m}, 0.32 \mathrm{~mm}$ of internal diameter), and helium was used as the carrier gas. The elemental analyses were performed on a Perkin-Elmer $2400 \mathrm{CHN}$ elemental analyser (São Paulo University/Brazil).

Methyl 4-alkylamino-6-oxo-7,7,7-trifluoro-4-heptenoates (3b and 3c)

To a stirred solution of methyl 7,7,7-trifluoro-4-methoxy-6-oxo-3-heptenoate 1 (5 mmol, $1.28 \mathrm{~g}$ ) in MeCN (5 $\mathrm{mL})$, kept at $25{ }^{\circ} \mathrm{C}$, was added benzylamine (5.1 mmol, $0.56 \mathrm{~mL}$ ) or phenethylamine (5.1 mmol, $0.62 \mathrm{~mL})$ in $\mathrm{MeCN}$ $(5 \mathrm{~mL})$. The mixture was stirred for $6 \mathrm{~h}$. The solvent was evaporated and the solid residue was dried under vacuum. The enaminones $\mathbf{3 b}$ and $\mathbf{3} \mathbf{c}$ were purified on silica-gel chromatographic column using chloroform-hexane (2:1) as eluent, yield refers to purified product.

3b:Yield $57 \%$; oil; Anal. Calcd. for $\mathrm{C}_{15} \mathrm{H}_{16} \mathrm{~F}_{3} \mathrm{NO}_{3}$ (315.29 g.mol ${ }^{-1}$ ): C, 57.14; H, 5.11, N, 4.44. Found: C, 58.10; H, 5.40; N, 4.65. ${ }^{1} \mathrm{H}$ NMR (400 MHz, $\left.\mathrm{CDCl}_{3}\right) \delta 2.56$ (m, H2), 2.67 (m, H3), 5.37 (s, H5), 3.69 (s, OMe), 4.61 (d, $\left.{ }^{2} \mathrm{~J}_{\mathrm{HH}} 6.0 \mathrm{~Hz}, \mathrm{CH}_{2}\right), 7.26-7.4(\mathrm{~m}, \mathrm{Ph}), 11.49(\mathrm{NH}, \mathrm{br}) .{ }^{13} \mathrm{C}$ NMR (100 MHz, $\left.\mathrm{CDCl}_{3}\right) \delta 176.2\left(\mathrm{q},{ }^{2} J_{\mathrm{CF}} 33 \mathrm{~Hz}, \mathrm{C} 6\right), 171.6$ (C4), 171.3 (C1), 135.9 (C1, Ph), 129.0 (C3 and C5, Ph), $128.1(\mathrm{C} 4, \mathrm{Ph}), 126.9$ ( C2 and C6, Ph), 117.5 (q, ${ }^{1} J_{\mathrm{CF}} 288$ $\mathrm{Hz}, \mathrm{C} 7), 87.5$ (C5), 51.9 (OMe), $47.2\left(-\mathrm{CH}_{2}-\right), 31.2$ (C2), 26.7 (C3). MS (EI, $70 \mathrm{eV}) \mathrm{m} / \mathrm{z}(\%): 315\left(\mathrm{M}^{+}, 29\right), 284$ (5), 246(68), 218 (36), 91 (100), 65 (75). 
3c: Yield $92 \%$; oil; Anal. Calcd. for $\mathrm{C}_{16} \mathrm{H}_{18} \mathrm{~F}_{3} \mathrm{NO}_{3}$ (329.32 g.mol ${ }^{-1}$ ): C, 58.36; H, 5.51, N, 4.25. Found: C, 57.70; H, 5.11; N, 4.44. ${ }^{1} \mathrm{H}$ NMR (400 MHz, $\left.\mathrm{CDCl}_{3}\right) \delta$ $2.93\left(\mathrm{t},{ }^{3} J_{\mathrm{HH}} 7.0 \mathrm{~Hz}, \mathrm{H} 2\right), 2.48\left(\mathrm{~m}, 4 \mathrm{H}, \mathrm{H} 3, \mathrm{CH}_{2}\right), 5.25(\mathrm{~s}$, $\mathrm{H} 5), 3.66$ (s, OMe), 3.64 (t, ${ }^{3} \mathrm{~J}_{\mathrm{HH}} 7.5 \mathrm{~Hz}, \mathrm{CH}_{2}$ ), $7.18-7.3$ (m, Ph), $11.25\left(\mathrm{NH}\right.$, br). ${ }^{13} \mathrm{C}$ NMR $\left(100 \mathrm{MHz}, \mathrm{CDCl}_{3}\right) \delta$ $175.6\left(\mathrm{q},{ }^{2} J_{\mathrm{CF}} 33 \mathrm{~Hz}, \mathrm{C} 6\right), 171.5$ (C4), 171.1 (C1), 137.2 $(\mathrm{C} 1, \mathrm{Ph}), 128.6(\mathrm{C} 3$ and $\mathrm{C} 5, \mathrm{Ph}), 128.5$ (C2 and $\mathrm{C} 6, \mathrm{Ph})$, 126.8 (C4, Ph), 117.5 (q, $\left.{ }^{1} J_{\mathrm{CF}} 288 \mathrm{~Hz}, \mathrm{C} 7\right), 87.1$ (C5), 51.7 (OMe), $44.9\left(\mathrm{NCH}_{2}-\right), 36.0\left(-\mathrm{CH}_{2} \mathrm{Ph}\right), 31.0(\mathrm{C} 2), 26.3(\mathrm{C} 3)$. MS (EI, $70 \mathrm{eV}): \mathrm{m} / \mathrm{z}(\%): 329\left(\mathrm{M}^{+}, 38\right), 298(23), 238(92)$, 206 (92), 178 (84), 104 (100), 77 (39).

Methyl 4-arylamino-6-oxo-7,7,7-trifluoro-4-heptenoates $(3, d-k)$

To a stirred solution of methyl 7,7,7-trifluoro-4methoxy-6-oxo-3-heptenoate $\mathbf{1}(5 \mathrm{mmol}, 1.28 \mathrm{~g}$ ) in $\mathrm{MeCN}$ $(5 \mathrm{~mL})$, was added the appropriate arylamine (Aldrich, $5.1 \mathrm{mmol})$ in $\mathrm{MeCN}(5 \mathrm{~mL})$. The mixture was refluxed for 6 to $24 \mathrm{~h}$. Then the solvent was evaporated and solid residue was dried under vacuum. Enaminones 3,d-k were recrystallised from hexane-chloroform (2:1), yield refers to purified product.

3d: Yield $76 \%$; mp 51-53 ${ }^{\circ} \mathrm{C}$; Anal. Calcd. for $\mathrm{C}_{14} \mathrm{H}_{14} \mathrm{~F}_{3} \mathrm{NO}_{3}\left(301.27{\mathrm{~g} . \mathrm{mol}^{-1}}^{-1}\right)$ C, 55.82; H, 4.68, N, 4.65 . Found: C, 56.07; H, 4.65; N, 4.35. ${ }^{1} \mathrm{H}$ NMR $(400 \mathrm{MHz}$, $\left.\mathrm{CDCl}_{3}\right) \delta 2.53\left(\mathrm{t},{ }^{3} \mathrm{~J}_{\mathrm{HH}} 7.5 \mathrm{~Hz}, \mathrm{H} 2\right), 2.72\left(\mathrm{t},{ }^{3} \mathrm{~J}_{\mathrm{HH}} 7.6 \mathrm{~Hz}, \mathrm{H} 3\right)$, 5.54 (s, H5), 3.64 (s, OMe), 7.14 - 7.5 (m, Ph), $12.6(\mathrm{NH}$, br). ${ }^{13} \mathrm{C} \mathrm{NMR}\left(100 \mathrm{MHz}, \mathrm{CDCl}_{3}\right) \delta 177.0\left(\mathrm{q},{ }^{2} J_{\mathrm{CF}} 33 \mathrm{~Hz}\right.$, C6), $171.5(\mathrm{C} 4), 170.0(\mathrm{C} 1), 136.4(\mathrm{C} 1, \mathrm{Ph}), 129.6$ (C3 and C5, Ph), 127.1, (C4, Ph), 125.7 (C2 and C6, Ph), 117.3 (q, $\left.{ }^{1} J_{\mathrm{CF}} 288 \mathrm{~Hz}, \mathrm{C} 7\right), 88.6(\mathrm{C} 5), 51.8(\mathrm{OMe}), 31.4(\mathrm{C} 2), 27.1$ (C3). MS (EI, $70 \mathrm{eV}): \mathrm{m} / \mathrm{z}(\%): 301\left(\mathrm{M}^{+}, 39\right), 270$ (19), 200 (82), 172 (78), 144 (48), 130 (49), 77 (100).

3e: Yield $92 \%$; mp 43-45 ${ }^{\circ} \mathrm{C}$; Anal. Calcd. for $\mathrm{C}_{15} \mathrm{H}_{16} \mathrm{~F}_{3} \mathrm{NO}_{3}\left(315.29 \mathrm{~g} \cdot \mathrm{mol}^{-1}\right)$ : C, 57.14; H, 5.11, N, 4.44. Found: C, 57.48; H, 4.99; N, 4.65. 'H NMR (400 MHz, $\left.\mathrm{CDCl}_{3}\right) \delta 2.52\left(\mathrm{t},{ }^{3} \mathrm{~J}_{\mathrm{HH}} 7.0 \mathrm{~Hz}, \mathrm{H} 2\right), 2.70\left(\mathrm{t},{ }^{3} J_{\mathrm{HH}} 7.2 \mathrm{~Hz}, \mathrm{H} 3\right)$, 5.51 (s, H5), 3.66 (s, OMe), $2.38\left(\mathrm{~s}, 4-\mathrm{CH}_{3}\right), 7.07$ (m, 2H, $\mathrm{Ph}), 7.23(\mathrm{~m}, 2 \mathrm{H}, \mathrm{Ph}), 12.5\left(\mathrm{NH}\right.$, br). ${ }^{13} \mathrm{C}$ NMR $(100 \mathrm{MHz}$, $\left.\mathrm{CDCl}_{3}\right) \delta 177.0\left(\mathrm{q},{ }^{2} J_{\mathrm{CF}} 33 \mathrm{~Hz}, \mathrm{C} 6\right), 170.6(\mathrm{C} 4), 170.0(\mathrm{C} 1)$, $137.9(\mathrm{C} 1, \mathrm{Ph}), 133.8(\mathrm{C} 4, \mathrm{Ph}), 130.2(\mathrm{C} 3$ and $\mathrm{C} 5, \mathrm{Ph})$, $125.6(\mathrm{C} 2$ and $\mathrm{C} 6, \mathrm{Ph}), 117.4\left(\mathrm{q},{ }^{1} J_{\mathrm{CF}} 288 \mathrm{~Hz}, \mathrm{C} 7\right), 88.5$ (C5), $51.9(\mathrm{OMe}), 31.4(\mathrm{C} 2), 27.1(\mathrm{C} 3), 20.9\left(4-\mathrm{CH}_{3}\right)$. MS (EI, $70 \mathrm{eV}): \mathrm{m} / z(\%): 315\left(\mathrm{M}^{+}, 61\right), 284(16), 228(25), 214$ (100), 186 (73), 158 (34), 144 (41), 91 (59).

3f: Yield $74 \%$; mp 52-54 ${ }^{\circ} \mathrm{C}$; Anal. Calcd. for $\mathrm{C}_{15} \mathrm{H}_{16} \mathrm{~F}_{3} \mathrm{NO}_{4}\left(331.29 \mathrm{~g} \cdot \mathrm{mol}^{-1}\right)$ : C, 54.38; H, 4.87, N, 4.23. Found: C, 54.25; H, 4.93; N, 3.87. ${ }^{1} \mathrm{H}$ NMR $(400 \mathrm{MHz}$, $\left.\mathrm{CDCl}_{3}\right) \delta 2.51\left(\mathrm{t},{ }^{3} J_{\mathrm{HH}} 7.8 \mathrm{~Hz}, \mathrm{H} 2\right), 2.66\left(\mathrm{t},{ }^{3} J_{\mathrm{HH}} 7.8 \mathrm{~Hz}, \mathrm{H} 3\right)$,
5.50 (s, H5), 3.66 (s, OMe), 3.83 (s, 4-OMe), 6.93 (m, 2H, $\mathrm{Ph}), 7.11$ (m, 2H, Ph), 12.4 (NH, br). ${ }^{13} \mathrm{C}$ NMR $(100 \mathrm{MHz}$, $\left.\mathrm{CDCl}_{3}\right) \delta 176.9\left(\mathrm{q},{ }^{2} J_{\mathrm{CF}} 33 \mathrm{~Hz}, \mathrm{C} 6\right), 171.6(\mathrm{C} 4), 170.5(\mathrm{C} 1)$, 159.1 (C4, Ph), 129.2.(C1, Ph), $127.2(\mathrm{C} 3$ and $\mathrm{C} 5, \mathrm{Ph})$, $117.4\left(\mathrm{q},{ }^{1} J_{\mathrm{CF}} 288 \mathrm{~Hz}, \mathrm{C} 7\right), 114.8(\mathrm{C} 2$ and $\mathrm{C} 6, \mathrm{Ph}), 88.4$ (C5), 55.5 (4-OMe), 51.9 (OMe), $31.5(\mathrm{C} 2), 27.2$ (C3). MS (EI, $70 \mathrm{eV}): m / z(\%): 331\left(\mathrm{M}^{+}, 100\right), 300(15), 272(24)$, 230 (78), 202 (38), 160 (27), 132 (13), 92 (15).

3g: Yield $98 \%$; mp 81-83 ${ }^{\circ} \mathrm{C}$; Anal. Calcd. for $\mathrm{C}_{14} \mathrm{H}_{13} \mathrm{ClF}_{3} \mathrm{NO}_{3}$ (335.71 g.mol ${ }^{-1}$ ): C, 50.09; H, 3.90, N, 4.71. Found: C, 50.18; H, 3.91; N, 4.97. ${ }^{1} \mathrm{H}$ NMR $(400 \mathrm{MHz}$, $\left.\mathrm{CDCl}_{3}\right) \delta 2.54\left(\mathrm{t},{ }^{3} J_{\mathrm{HH}} 7.2 \mathrm{~Hz}, \mathrm{H} 2\right), 2.70\left(\mathrm{t},{ }^{3} J_{\mathrm{HH}} 7.2 \mathrm{~Hz}\right.$, H3), 5.55 (s, H5), 3.66 (s, OMe), 7.16 (m, 2H, Ph), 7.40 (m, 2H, Ph), 12.5 (NH, br). ${ }^{13} \mathrm{C}$ NMR (100 MHz, $\left.\mathrm{CDCl}_{3}\right)$ $\delta 177.3\left(\mathrm{q},{ }^{2} J_{\mathrm{CF}} 33 \mathrm{~Hz}, \mathrm{C} 6\right), 171.4(\mathrm{C} 4), 169.8(\mathrm{C} 1), 135.0$ $(\mathrm{C} 1, \mathrm{Ph}), 133.5(\mathrm{C} 4, \mathrm{Ph}), 129.7(\mathrm{C} 3$ and $\mathrm{C} 5, \mathrm{Ph}), 127.0$ ( $\mathrm{C} 2$ and $\mathrm{C} 6, \mathrm{Ph}), 117.2\left(\mathrm{q},{ }^{1} J_{\mathrm{CF}} 288 \mathrm{~Hz}, \mathrm{C} 7\right), 88.9$ (C5), 51.8 (OMe), $31.3(\mathrm{C} 2), 26.9(\mathrm{C} 3)$. MS (EI, $70 \mathrm{eV}): \mathrm{m} / z$ (\%): 335 $\left(\mathrm{M}^{+}, 31\right), 304$ (14), 276 (18), 234 (100), 206 (96), 178 (60), 143 (39), 111 (81), 75 (82).

3h: Yield $95 \%$; mp 77-79 ${ }^{\circ} \mathrm{C}$; Anal. Calcd. for $\mathrm{C}_{14} \mathrm{H}_{13} \mathrm{BrF}_{3} \mathrm{NO}_{3}\left(380.16\right.$ g.mol ${ }^{-1}$ ): C, 44.23; H, 3.45, N, 3.68. Found: C, 44.37; H, 3.44; N, 3.57. ${ }^{1} \mathrm{H}$ NMR $(400 \mathrm{MHz}$, $\left.\mathrm{CDCl}_{3}\right) \delta=2.54\left(\mathrm{t},{ }^{3} J_{\mathrm{HH}}=7.2 \mathrm{~Hz}, \mathrm{H} 2\right), 2.70\left(\mathrm{t},{ }^{3} J_{\mathrm{HH}}=7.2\right.$ $\mathrm{Hz}, \mathrm{H} 3), 5.55$ (s, H5), 3.67 (s, OMe), 7.10 (m, 2H, Ph), 7.56 $(\mathrm{m}, 2 \mathrm{H}, \mathrm{Ph}), 12.5(\mathrm{NH}, \mathrm{br}){ }^{13} \mathrm{C} \mathrm{NMR}\left(100 \mathrm{MHz}, \mathrm{CDCl}_{3}\right)$ $\delta 177.3\left(\mathrm{q},{ }^{2} J_{\mathrm{CF}} 34 \mathrm{~Hz}, \mathrm{C} 6\right), 171.4(\mathrm{C} 4), 169.7$ (C1), 135.4 (C1, Ph), 132.7 (C3 and $\mathrm{C} 5, \mathrm{Ph}), 127.3$ (C2 and $\mathrm{C} 6, \mathrm{Ph})$, $121.4(\mathrm{C} 4, \mathrm{Ph}), 117.1$ (q, $\left.{ }^{1} J_{\mathrm{CF}} 288 \mathrm{~Hz}, \mathrm{C} 7\right), 88.9$ (C5), 51.9 (OMe), $31.2(\mathrm{C} 2), 26.9(\mathrm{C} 3)$. MS (EI, $70 \mathrm{eV}): \mathrm{m} / z$ (\%): 380 $\left(\mathrm{M}^{+}, 69\right), 348$ (21), 310 (34), 278 (100), 250 (52), 231 (57), 199 (61), 172 (70), 143 (86), 76 (77).

3i: Yield $95 \%$; mp 68-70 ${ }^{\circ} \mathrm{C}$; Anal. Calcd. for $\mathrm{C}_{12} \mathrm{H}_{13} \mathrm{~F}_{3} \mathrm{~N}_{2} \mathrm{O}_{4}\left(306.24{\mathrm{~g} . \mathrm{mol}^{-1}}^{-1}\right)$ C, $47.06 ; \mathrm{H}, 4.28, \mathrm{~N}, 9.15$. Found: C, 46.97; H, 4.19; N, 8.98. ${ }^{1} \mathrm{H}$ NMR (400 MHz, $\left.\mathrm{CDCl}_{3}\right) \delta 2.72\left(\mathrm{t},{ }^{3} J_{\mathrm{HH}} 7.6 \mathrm{~Hz}, \mathrm{H} 2\right), 3.13\left(\mathrm{t},{ }^{3} J_{\mathrm{HH}} 7.6 \mathrm{~Hz}, \mathrm{H} 3\right)$, 5.68 (s, H5), 3.70 (s, OMe), 2.43 (s, 3- $\mathrm{CH}_{3}$, isox), 5.98 (s, $\mathrm{H} 5$, isox), $12.4(\mathrm{NH}, \mathrm{br}) .{ }^{13} \mathrm{C}$ NMR $\left(100 \mathrm{MHz}, \mathrm{CDCl}_{3}\right) \delta$ 178.5 (q, $\left.{ }^{2} J_{\mathrm{CF}} 34 \mathrm{~Hz}, \mathrm{C} 6\right), 171.6$ (C4), 170.7 (C1), 168.3 (C5, isox), 157.6 (C3, isox), 116.6 (q, $\left.{ }^{1} J_{\mathrm{CF}} 288 \mathrm{~Hz}, \mathrm{C} 7\right)$, 96.7 (C4, isox), 92.2 (C5), 51.5 (OMe), 31.3 (C2), 29.4 (C3), 11.9 (3-Me, isox). MS (EI, $70 \mathrm{eV}): \mathrm{m} / z(\%): 306\left(\mathrm{M}^{+}\right.$, 9), 275 (18), 237 (100), 278 (100), 205 (60), 149 (43), 135 (47), 119 (46), 68 (58).

3j:Yield 95\%; mp 65-68 ${ }^{\circ} \mathrm{C}$; Anal. Calcd. for $\mathrm{C}_{13} \mathrm{H}_{13} \mathrm{~F}_{3} \mathrm{~N}_{2} \mathrm{O}_{3}$ (302.26 g.mol ${ }^{-1}$ ): C, 51.66; H, 4.34, N, 9.27. Found: C, 51.41; $\mathrm{H}, 4.18 ; \mathrm{N}, 9.30 .{ }^{1} \mathrm{H} \mathrm{NMR}\left(400 \mathrm{MHz}, \mathrm{CDCl}_{3}\right) \delta 2.76\left(\mathrm{t},{ }^{3} \mathrm{~J}_{\mathrm{HH}}\right.$ $7.2 \mathrm{~Hz}, \mathrm{H} 2), 3.33$ (t, $\left.{ }^{3} \mathrm{~J}_{\mathrm{HH}} 7.2 \mathrm{~Hz}, \mathrm{H} 3\right), 5.61$ (s, H5), 3.70 (s, $\mathrm{OMe}$ ), 7.0, 7.1, 7.7, 8.4 (2-pyridyl), 12.9 (NH, br). ${ }^{13} \mathrm{C} \mathrm{NMR}$ $\left(100 \mathrm{MHz}, \mathrm{CDCl}_{3}\right) \delta 177.8\left(\mathrm{q},{ }^{2} J_{\mathrm{CF}} 34 \mathrm{~Hz}, \mathrm{C} 6\right), 172.1(\mathrm{C} 4)$, 168.7 (C1), 151.1 (C2, pyr), 148.3 (C6, pyr), 138.4 (C4, pyr), 
120.2 (C3, pyr), 115.5 (C5, pyr), 117.0 (q, $\left.{ }^{1} J_{\mathrm{CF}} 288 \mathrm{~Hz}, \mathrm{C} 7\right)$, 92.5 (C5), 51.1 (OMe), 32.3 (C2), 29.5 (C3). MS (EI, $70 \mathrm{eV})$ : $m / z$ (\%): $271\left(\mathrm{M}^{+}-\mathrm{OMe}, 34\right), 233$ (21), 205 (100), 173 (98), 131 (88), 96 (18), 78 (97), 51 (38).

3k: Yield $95 \%$; mp $65-68{ }^{\circ} \mathrm{C}$; Anal. Calcd. for $\mathrm{C}_{14} \mathrm{H}_{15} \mathrm{~F}_{3} \mathrm{~N}_{2} \mathrm{O}_{3}\left(316.28\right.$ g.mol $\left.^{-1}\right)$ : C, 53.17; H, 4.78, N, 8.86. Found: C, 55.00; H, 5.28; N, 9.90. ${ }^{1} \mathrm{H}$ NMR (400 MHz, $\left.\mathrm{CDCl}_{3}\right) \delta 2.50\left(\mathrm{t},{ }^{3} \mathrm{~J}_{\mathrm{HH}} 7.5 \mathrm{~Hz}, \mathrm{H} 2\right), 2.66\left(\mathrm{t},{ }^{3} \mathrm{~J}_{\mathrm{HH}} 7.5 \mathrm{~Hz}\right.$, H3), 5.47 (s, H5), 3.66 (s, OMe), 6.68 (m, 2H, Ph), 6.96 (m, 2H, Ph), 13.2 (NH, br). ${ }^{13} \mathrm{C}$ NMR (100 MHz, $\mathrm{CDCl}_{3}$ ) $\delta 177.6\left(\mathrm{q},{ }^{2} \mathrm{~J}_{\mathrm{CF}} 34 \mathrm{~Hz}, \mathrm{C} 6\right), 171.7$ (C4), 170.8 (C1), 146.3 (C4, Ph), 126.9 (C3, C4 and C5, Ph), 115.4 (C2 and C6, $\mathrm{Ph}$ ), 88.2 (C5), 31.5 (C2), 27.2 (C3). MS (EI, $70 \mathrm{eV}): \mathrm{m} / \mathrm{z}$ (\%): $316\left(\mathrm{M}^{+}, 100\right), 285$ (10), 257 (15), 215 (36), 187 (33), 173 (32), 92 (19).

\section{Methyl 7,7,7-trichloro-4-alkylamino-6-oxo-4-heptenoates} (4a and $\mathbf{4 c})$

To a stirred solution of methyl 7,7,7-trichloro-4-methoxy-6-oxo-3-heptenoate 2 (5 mmol, $1.52 \mathrm{~g}$ ) in $\mathrm{CHCl}_{3}$ (5 $\mathrm{mL}$ ), kept at $25{ }^{\circ} \mathrm{C}$, was added n-propylamine (Aldrich, 18570-1, $5.1 \mathrm{mmol}, 0.42 \mathrm{~mL}$ ) or phenethylamine (Aldrich, 24095-8, $5.1 \mathrm{mmol}, 0.62 \mathrm{~mL}$ ) in $\mathrm{CHCl}_{3}(5 \mathrm{~mL})$. The mixture was stirred for $2 \mathrm{~h}$. Then the solvent was evaporated and solid residue was dried under vacuum. The enaminones 4a and $\mathbf{4} \mathbf{c}$ were purified on silica-gel chromatographic column using chloroform-hexane $(2: 1)$ as eluent, yield refers to purified product.

4a: Yield $94 \%$; Oil; Anal. Calcd. for $\mathrm{C}_{11} \mathrm{H}_{16} \mathrm{Cl}_{3} \mathrm{NO}_{3}$ (316.61 g. $\left.\mathrm{mol}^{-1}\right)$ : C, 41.73; H, 5.09, N, 4.42. Found: C, 41.39; H, 4.95; N, 4.30. ${ }^{1} \mathrm{H} \mathrm{NMR}\left(400 \mathrm{MHz}, \mathrm{CDCl}_{3}\right) \delta$ 2.69 (m, H2 and H3), 5.67 (s, H5), 3.73 (s, OMe), 3.36 $\left(\mathrm{t}, \mathrm{CH}_{2}\right), 1.72\left(\mathrm{q}, \mathrm{CH}_{2}\right), 1.05\left(\mathrm{t}, \mathrm{CH}_{3}\right), 12.8(\mathrm{NH}, \mathrm{br}) .{ }^{13} \mathrm{C}$ NMR (100 MHz, $\mathrm{CDCl}_{3}$ ) $\delta 180.1$ (C6), 171.6 (C4), 170.3 (C1), 96.9 (C7), 84.2 (C5) 51.8 (OMe), 44.9 ( $\left.\mathrm{NCH}_{2}{ }^{-}\right)$, 31.2 (C2), 27.0 (C3), $22.7\left(-\mathrm{CH}_{2}-\right), 11.1$ (Me). MS (EI, $70 \mathrm{eV}): \mathrm{m} / z(\%): 318\left(\mathrm{M}^{+}+2,10\right), 316\left(\mathrm{M}^{+}, 10\right), 252(39)$, 198 (100), 166 (36).

4c: Yield $98 \%$; Oil; Anal. Calcd. for $\mathrm{C}_{16} \mathrm{H}_{18} \mathrm{Cl}_{3} \mathrm{NO}_{3}$ (378.69 g. $\left.\mathrm{mol}^{-1}\right)$ : C, 50.75; H, 4.79, N, 3.70. Found: C, 52.0; H, 4.97; N, 4.0. ${ }^{1} \mathrm{H}$ NMR (400 MHz, $\left.\mathrm{CDCl}_{3}\right) \delta 2.53$ (t, $\left.{ }^{3} J_{\mathrm{HH}} 7.2 \mathrm{~Hz}, \mathrm{H} 2\right), 2.95\left(\mathrm{t},{ }^{3} J_{\mathrm{HH}} 7.2 \mathrm{~Hz}, \mathrm{H} 3\right), 5.64$ (s, H5), 3.69 (s, OMe), $2.53\left(\mathrm{CH}_{2}\right), 3.61\left(\mathrm{CH}_{2}\right), 7.2-7.33$ (Ph), $12.8\left(\mathrm{NH}\right.$, br). ${ }^{13} \mathrm{C}$ NMR $\left(100 \mathrm{MHz}, \mathrm{CDCl}_{3}\right) \delta 180.3$ (C6), 171.6 (C4), 170.2 (C1), 137.3 (C1, Ph), 128.6 (C3 and $\mathrm{C} 5, \mathrm{Ph}), 128.5(\mathrm{C} 4, \mathrm{Ph}), 126.8(\mathrm{C} 2$ and $\mathrm{C} 6, \mathrm{Ph}), 96.9$ (C7), 84.5 (C5), 51.9 (OMe), $45.0\left(\mathrm{NCH}_{2}-\right), 36.1\left(-\mathrm{CH}_{2} \mathrm{Ph}\right)$, 31.2 (C2), 26.8 (C3). MS (EI, $70 \mathrm{eV}): \mathrm{m} / \mathrm{z}(\%): 381\left(\mathrm{M}^{+}+\right.$ 2, 13), $379\left(\mathrm{M}^{+}, 10\right), 314$ (44), 286 (50), 260 (100), 228 (23), 105 (100), 77 (50).
Methyl 7,7,7-trichloro-4-arylamino-6-oxo-4-heptenoates (4d-4i)

To a stirred solution of methyl 7,7,7-trichloro-4methoxy-6-oxo-3-heptenoate 2 ( $5 \mathrm{mmol}, 1.52 \mathrm{~g}$ ) in $\mathrm{MeCN}$ $(5 \mathrm{~mL})$, kept at $25^{\circ} \mathrm{C}$, was added appropriate arylamine (Aldrich, $5.1 \mathrm{mmol}$ ) in $\mathrm{MeCN}(5 \mathrm{~mL})$. The mixture was refluxed for 2 to $6 \mathrm{~h}$. The solvent was evaporated and the solid residue was dried under reduced pressure. The enaminones $\mathbf{4 d} \mathbf{d} \mathbf{i}$, $\mathbf{4 k}$ were recrystallised from hexane: $\mathrm{CHCl}_{3}$ (2:1), yield refers to purified product.

4d: Yield $73 \%$; mp 79-82 ${ }^{\circ} \mathrm{C}$; Anal. Calcd. for $\mathrm{C}_{14} \mathrm{H}_{14} \mathrm{Cl}_{3} \mathrm{NO}_{3}\left(350.63\right.$ g.mol $\left.{ }^{-1}\right)$ : C, 47.96; H, 4.02, N, 3.99 . Found: C, 48.0; H, 4.50; N, 3.90. ${ }^{1} \mathrm{H}$ NMR (400 MHz, $\left.\mathrm{CDCl}_{3}\right) \delta 2.52\left(\mathrm{t},{ }^{3} J_{\mathrm{HH}} 7.0 \mathrm{~Hz}, \mathrm{H} 2\right), 2.75\left(\mathrm{t},{ }^{3} J_{\mathrm{HH}} 7.0 \mathrm{~Hz}\right.$, H3), 5.88 (s, H5), 3.66 (s, OMe), 7.2 - $7.44(\mathrm{Ph}), 13.8$ (NH, br). ${ }^{13} \mathrm{C} \mathrm{NMR}\left(100 \mathrm{MHz}, \mathrm{CDCl}_{3}\right) \delta 181.4$ (C6), $171.6(\mathrm{C} 4)$, $168.8(\mathrm{C} 1), 136.8(\mathrm{C} 1, \mathrm{Ph}), 129.6(\mathrm{C} 3$ and $\mathrm{C} 5, \mathrm{Ph}), 127.5$ (C4, Ph), 125.7 (C2 and C6, Ph), 96.9 (C7), 86.5 (C5), 51.9 (OMe), 31.6 (C2), 27.5 (C3). MS (EI, $70 \mathrm{eV}): \mathrm{m} / \mathrm{z}(\%): 352$ $\left(\mathrm{M}^{+}+2,<5\right), 350\left(\mathrm{M}^{+}, 10\right), 232$ (85), 200 (100), 172 (61), 144 (27), 77 (60).

4e: Yield $76 \%$; mp 53-55 ${ }^{\circ} \mathrm{C}$; Anal. Calcd. for $\mathrm{C}_{14} \mathrm{H}_{14} \mathrm{Cl}_{3} \mathrm{NO}_{3}\left(364.66\right.$ g.mol $\left.{ }^{-1}\right)$ : C, 49.41; H, 4.42, N, 3.84 . Found: C, 49.90; H, 5.0; N, 3.55. ${ }^{1} \mathrm{H}$ NMR (400 MHz, $\left.\mathrm{CDCl}_{3}\right) \delta 2.52\left(\mathrm{t},{ }^{3} \mathrm{~J}_{\mathrm{HH}} 7.7 \mathrm{~Hz}, \mathrm{H} 2\right), 2.73\left(\mathrm{t},{ }^{3} \mathrm{~J}_{\mathrm{HH}} 7.5 \mathrm{~Hz}, \mathrm{H} 3\right)$, 5.86 (s, H5), 3.65 (s, OMe), 2.37 (4-Me), 7.1 (3H, Ph), 7.3 $(2 \mathrm{H}, \mathrm{Ph}), 13.6(\mathrm{NH}, \mathrm{br}) .{ }^{13} \mathrm{C} \mathrm{NMR}\left(100 \mathrm{MHz}, \mathrm{CDCl}_{3}\right) \delta$ 181.2 (C6), 171.7 (C4), 169.2 (C1), 137.6 (C1, Ph), 134.1 (C3 and $\mathrm{C} 5, \mathrm{Ph}), 130.1(\mathrm{C} 4, \mathrm{Ph}), 125.5$ (C2 and $\mathrm{C} 6, \mathrm{Ph})$, 96.9 (C7), 86.2 (C5) 51.9 (OMe), 31.7 (C2), 27.5 (C3), 20.9 (4-Me). MS (EI, $70 \mathrm{eV}): \mathrm{m} / \mathrm{z}(\%): 367\left(\mathrm{M}^{+}+2,7\right), 365\left(\mathrm{M}^{+}\right.$, 7), 246 (70), 214 (100), 186 (47), 158 (14), 91 (39).

4f: Yield $70 \%$; mp $79-81{ }^{\circ} \mathrm{C}$; Anal. Calcd. for $\mathrm{C}_{15} \mathrm{H}_{16} \mathrm{Cl}_{3} \mathrm{NO}_{4}\left(380.66\right.$ g.mol $\left.{ }^{-1}\right): \mathrm{C}, 47.33 ; \mathrm{H}, 4.24, \mathrm{~N}, 3.68$. Found: C, 47.10; H, 4.20; N, 4.0. ${ }^{1} \mathrm{H}$ NMR (400 MHz, $\left.\mathrm{CDCl}_{3}\right) \delta 2.52\left(\mathrm{t},{ }^{3} J_{\mathrm{HH}} 7.5 \mathrm{~Hz}, \mathrm{H} 2\right), 2.70\left(\mathrm{t},{ }^{3} \mathrm{~J}_{\mathrm{HH}} 7.5 \mathrm{~Hz}\right.$, H3), 5.86 (s, H5), 3.66 (s, OMe), 3.84 (4-OMe), 6.9 (3H, $\mathrm{Ph}), 7.13$ (2H, Ph), 13.6 (NH, br). ${ }^{13} \mathrm{C}$ NMR (100 MHz, $\mathrm{CDCl}_{3}$ ) $\delta 181.2$ (C6), 171.7 (C4), 169.5 (C1), 158.9 (C4, $\mathrm{Ph}), 129.2(\mathrm{C} 1, \mathrm{Ph}), 127.2(\mathrm{C} 3$ and $\mathrm{C} 5, \mathrm{Ph}), 114.7$ (C2 and C6, Ph), 97.0 (C7), 86.0 (C5), 55.4 (4-OMe), 51.9 (OMe), 31.7 (C2), 27.5 (C3). MS (EI, $70 \mathrm{eV}): \mathrm{m} / \mathrm{z}$ (\%): $381\left(\mathrm{M}^{+}+2,15\right), 379\left(\mathrm{M}^{+}, 14\right), 262$ (75), 230 (100), 202 (28), 174 (10), 77 (16).

4g: Yield $88 \%$; mp 105-107 ${ }^{\circ} \mathrm{C}$; Anal. Calcd. for $\mathrm{C}_{14} \mathrm{H}_{13} \mathrm{Cl}_{4} \mathrm{NO}_{3}\left(385.08\right.$ g.mol $\left.{ }^{-1}\right): \mathrm{C}, 43.67 ; \mathrm{H}, 3.40, \mathrm{~N}, 3.64$. Found: C, 44.87; H, 4.10; N, 3.90. ' H NMR (400 MHz, $\left.\mathrm{CDCl}_{3}\right) \delta 2.54\left(\mathrm{t},{ }^{3} J_{\mathrm{HH}} 7.8 \mathrm{~Hz}, \mathrm{H} 2\right), 2.73\left(\mathrm{t},{ }^{3} J_{\mathrm{HH}} 7.6 \mathrm{~Hz}\right.$, H3), 5.89 (s, H5), 3.66 (s, OMe), 7.15 (3H, Ph), $7.40(2 \mathrm{H}$, $\mathrm{Ph}), 13.4$ (NH, br). ${ }^{13} \mathrm{C}$ NMR (100 MHz, $\left.\mathrm{CDCl}_{3}\right) \delta 181.7$ 
(C6), 171.5 (C4), 168.6 (C1), 135.4 (C1, Ph), 133.3 (C3 and $\mathrm{C} 5, \mathrm{Ph}), 129.8(\mathrm{C} 4, \mathrm{Ph}), 127.0(\mathrm{C} 2$ and $\mathrm{C} 6, \mathrm{Ph}), 96.8$ (C7), 86.9 (C5), 52.0 (OMe), 31.6 (C2), 27.4 (C3). MS (EI, $70 \mathrm{eV}): \mathrm{m} / \mathrm{z}(\%): 385\left(\mathrm{M}^{+}+2,10\right), 383\left(\mathrm{M}^{+}, 6\right), 266(70)$, 234 (100), 206 (40), 178 (10), 111 (10).

4h: Yield $85 \%$; mp 97-100 ${ }^{\circ} \mathrm{C}$; Anal. Calcd. for $\mathrm{C}_{14} \mathrm{H}_{13} \mathrm{BrCl}_{3} \mathrm{NO}_{3}\left(429.53\right.$ g.mol $\left.{ }^{-1}\right): \mathrm{C}, 39.15 ; \mathrm{H}, 3.05, \mathrm{~N}$, 3.26. Found: C, 39.17; H, 3.10; N, 3.50. ${ }^{1} \mathrm{H}$ NMR (400 $\left.\mathrm{MHz}, \mathrm{CDCl}_{3}\right) \delta 2.54\left(\mathrm{t},{ }^{3} J_{\mathrm{HH}} 7.5 \mathrm{~Hz}, \mathrm{H} 2\right), 2.74\left(\mathrm{t},{ }^{3} \mathrm{~J}_{\mathrm{HH}} 7.6\right.$ $\mathrm{Hz}, \mathrm{H} 3), 5.89$ (s, H5), 3.67 (s, OMe), 7.1 (3H, Ph), 7.56 (2H, Ph), 13.0 (NH, br). $\left.{ }^{13} \mathrm{C} \mathrm{NMR} \mathrm{(100} \mathrm{MHz,} \mathrm{CDCl}_{3}\right) \delta$ 181.6 (C6), 171.5 (C4), 168.4 (C1), 135.4 (C1, Ph), 133.3 (C3 and C5, Ph), $129.8(\mathrm{C} 4, \mathrm{Ph}), 127.0$ (C2 and C6, Ph), 96.7 (C7), 86.9 (C5), 52.0 (OMe), 31.5 (C2), 27.4 (C3). MS (EI, $70 \mathrm{eV}): \mathrm{m} / \mathrm{z}(\%): 430\left(\mathrm{M}^{+}+2,16\right), 428\left(\mathrm{M}^{+}, 10\right)$, 310 (86), 278 (100), 250 (30), 231 (70).

4k: Yield $90 \%$; mp 114-116 ${ }^{\circ} \mathrm{C}$; Anal. Calcd. for $\mathrm{C}_{14} \mathrm{H}_{15} \mathrm{Cl}_{3} \mathrm{~N}_{2} \mathrm{O}_{3}\left(366.65\right.$ g.mol ${ }^{-1}$ ): C, 45.99; H, 4.13, N, 7.66. Found: C, 47.17; H, 4.83; N, 8.20. ${ }^{1} \mathrm{H}$ NMR (400 $\left.\mathrm{MHz}, \mathrm{CDCl}_{3}\right) \delta 2.57\left(\mathrm{t},{ }^{3} J_{\mathrm{HH}} 7.0 \mathrm{~Hz}, \mathrm{H} 2\right), 2.74\left(\mathrm{t},{ }^{3} \mathrm{~J}_{\mathrm{HH}}\right.$ $7.2 \mathrm{~Hz}, \mathrm{H} 3), 5.85$ (s, H5), 3.60 (s, OMe), 6.75 (3H, Ph), $7.0(2 \mathrm{H}, \mathrm{Ph}), 13.8$ (NH, br). ${ }^{13} \mathrm{C}$ NMR (100 MHz, $\left.\mathrm{CDCl}_{3}\right)$ $\delta 181.7$ (C6), 173.3 (C4), 172.3 (C1), 149.6 (C4, Ph), $128.6(\mathrm{C} 3$ and $\mathrm{C} 5, \mathrm{Ph}), 127.5(\mathrm{C} 1, \mathrm{Ph}), 116.4(\mathrm{C} 2$ and C6, Ph), 99.1 (C7), 86.6 (C5), 52.9 (OMe), 33.0 (C2), 29.0 (C3). MS (EI, $70 \mathrm{eV}): \mathrm{m} / z(\%): 366\left(\mathrm{M}^{+}+2,16\right)$, $364\left(\mathrm{M}^{+}, 15\right), 247$ (46), 215 (100), 187 (38), 173 (50), 159 (12), 92 (16).

1-[Alkyl(aryl)]-5-(3,3,3-trifluoro[chloro]-2-oxopropylidene)pyrrolidin-2-ones $(\mathbf{5 , 6 )}$

To a stirred solution of appropriate methyl 7,7,7-trihalo4-alkyl(aryl)amino-6-oxo-3-heptenoate (3 or 4, 2 mmol) in $\mathrm{MeCN}(5 \mathrm{~mL})$, kept at $25^{\circ} \mathrm{C}$, was added Et $\mathrm{N}$ ( 2 mmol, 0.3 $\mathrm{mL})$ in $\mathrm{MeCN}(1 \mathrm{~mL})$. The mixture was refluxed for 1 to $24 \mathrm{~h}$ and the solvent was removed under reduced pressure. The residue was crystallised from hexane, yield refers to purified product.

5b: Yield $69 \%$; mp 96-98 ${ }^{\circ} \mathrm{C}$; Anal. Calcd. for $\mathrm{C}_{14} \mathrm{H}_{12} \mathrm{~F}_{3} \mathrm{NO}_{2}\left(283.25\right.$ g.mol $\left.{ }^{-1}\right): \mathrm{C}, 59.37 ; \mathrm{H}, 4.27, \mathrm{~N}, 4.94$. Found: C, 60.10; H, 4.01; N, 5.03. ${ }^{1} \mathrm{H}$ NMR (400 MHz, $\left.\mathrm{CDCl}_{3}\right) \delta 2.67$ (m, H3), 3.33 (m, H4), 5.89 (s, CH), 4.79 $\left(\mathrm{s}, \mathrm{CH}_{2}\right), 7.23(3 \mathrm{H}, \mathrm{Ph}), 7.4(2 \mathrm{H}, \mathrm{Ph}) .{ }^{13} \mathrm{C} \mathrm{NMR}(100 \mathrm{MHz}$, $\left.\mathrm{CDCl}_{3}\right) \delta 178.6\left({ }^{2} J_{\mathrm{CF}} 34 \mathrm{~Hz}, \mathrm{C} 2\right.$, propylidene), 177.1 (C5, pyrrolidin-2-one), 167.8 (C2, pyrrolidin-2-one), 133.9 (C1, $\mathrm{Ph}), 128.9(\mathrm{C} 4, \mathrm{Ph}), 128.1$ (C2 and C6, Ph), 127.3 (C3 and $\mathrm{C} 5, \mathrm{Ph}), 116.3$ ( ${ }^{1} J_{\mathrm{CF}} 292 \mathrm{~Hz}, \mathrm{C} 3$, propylidene), 92.0 (C1, propylidene), 44.5 (- $\left.\mathrm{CH}_{2}-\right), 27.2$ (C4, pyrrolidin-2-one), 26.6 (C3, pyrrolidin-2-one). MS (EI, $70 \mathrm{eV}): \mathrm{m} / \mathrm{z}(\%): 283$ $\left(\mathrm{M}^{+}, 5\right), 214$ (28), 186 (17), 91 (100), 65 (22). 5c: Yield $87 \%$; mp 103-105 ${ }^{\circ} \mathrm{C}$; Anal. Calcd. for $\mathrm{C}_{15} \mathrm{H}_{14} \mathrm{~F}_{3} \mathrm{NO}_{2}\left(297.28\right.$ g.mol ${ }^{-1}$ ): C, 60.61; H, 4.75, N, 4.71. Found: C, 60.41; H, 4.60; N, 4.57. ${ }^{1} \mathrm{H}$ NMR (400 MHz, $\left.\mathrm{CDCl}_{3}\right) \delta 2.56$ (m, H3, pyrrolidin-2-one), 3.29 (m, H4, pyrrolidin-2-one), 5.78 (s, H1, propylidene), $2.88\left(\mathrm{CH}_{2}\right)$, $3.82\left(\mathrm{CH}_{2}\right), 7.1-7.4(\mathrm{Ph}) .{ }^{13} \mathrm{C} \mathrm{NMR}\left(100 \mathrm{MHz}, \mathrm{CDCl}_{3}\right) \delta$ $178.6\left({ }^{2} J_{\mathrm{CF}} 34 \mathrm{~Hz}, \mathrm{C} 2\right.$, propylidene), 176.8 (C5, pyrrolidin2-one), 168.2 (C2, pyrrolidin-2-one), $137.0(\mathrm{C} 1, \mathrm{Ph}), 128.7$ (C3 and C5, Ph), 128.6 (C2 and C6, Ph), 127 (C4, Ph), 116.4 ( ${ }^{1} J_{\mathrm{CF}} 292 \mathrm{~Hz}, \mathrm{C} 3$, propylidene), 91.0 (C1, propylidene), 42.3 $\left(\mathrm{NCH}_{2}\right), 32.6\left(\mathrm{CH}_{2} \mathrm{Ph}\right), 26.7(\mathrm{C} 3$, pyrrolidin-2-one $), 27.1$ (C4, pyrrolidin-2-one). MS (EI, $70 \mathrm{eV}): \mathrm{m} / \mathrm{z}(\%): 297\left(\mathrm{M}^{+}\right.$, 5), 228 (24), 178 (10), 104 (100), 77 (13).

5d: Yield $77 \%$; mp 146-149 ${ }^{\circ} \mathrm{C}$; Anal. Calcd. for $\mathrm{C}_{13} \mathrm{H}_{10} \mathrm{~F}_{3} \mathrm{NO}_{2}\left(269.23\right.$ g.mol $\left.{ }^{-1}\right)$ : C, 58.00; H, 3.74, N, 5.20. Found: C, 57.78; H, 3.81; N, 4.90. ${ }^{1} \mathrm{H}$ NMR (400 MHz, $\left.\mathrm{CDCl}_{3}\right) \delta 2.83$ (m, H3), 3.53 (m, H4), 5.66 (s, CH), 7.2 $(3 \mathrm{H}, \mathrm{Ph}), 7.5(2 \mathrm{H}, \mathrm{Ph}) .{ }^{13} \mathrm{C} \mathrm{NMR}\left(100 \mathrm{MHz}, \mathrm{CDCl}_{3}\right) \delta 179.2$ $\left({ }^{2} J_{\mathrm{CF}} 34 \mathrm{~Hz}, \mathrm{C} 2\right.$, propylidene), 176.5 (C5, pyrrolidin-2-one), 169.2 (C2, pyrrolidin-2-one), 133.2 (C1, Ph), 130.1 (C3 and $\mathrm{C} 5, \mathrm{Ph}), 129.8$ (C2 and C5, Ph), $127.1(\mathrm{C} 4, \mathrm{Ph}), 116.4\left({ }^{1} J_{\mathrm{CF}}\right.$ $292 \mathrm{~Hz}, \mathrm{C} 3$, propylidene), 92.7 (C1, propylidene), 27.8 (C4, pyrrolidin-2-one), 26.9 (C3, pyrrolidin-2-one). MS (EI, 70 $\mathrm{eV}): m / z$ (\%): 269 (M+13), 200 (100), 172 (68), 144 (29), 77 (69), 51 (41).

5e: Yield $86 \%$; mp 129-131 ${ }^{\circ} \mathrm{C}$; Anal. Calcd. for $\mathrm{C}_{14} \mathrm{H}_{12} \mathrm{~F}_{3} \mathrm{NO}_{2}\left(283.25 \mathrm{~g} \cdot \mathrm{mol}^{-1}\right)$ : C, 59.37; H, 4.27, N, 4.94 . Found: C, 59.28; H, 4.38; N, 4.79. ${ }^{1} \mathrm{H}$ NMR (400 MHz, $\left.\mathrm{CDCl}_{3}\right) \delta 2.80$ (m, H3, pyrrolidin-2-one), 3.50 (m, H4, pyrrolidin-2-one), 5.67 (s, H1, propylidene), 2.43 (4-Me), $7.06(3 \mathrm{H}, \mathrm{Ph}), 7.34(2 \mathrm{H}, \mathrm{Ph}) .{ }^{13} \mathrm{C} \mathrm{NMR}\left(100 \mathrm{MHz}, \mathrm{CDCl}_{3}\right) \delta$ $179.0\left({ }^{2} J_{\mathrm{CF}} 34 \mathrm{~Hz}, \mathrm{C} 2\right.$, propylidene), 176.7 (C5, pyrrolidin2-one), 169.5 (C2, pyrrolidin-2-one), $139.9(\mathrm{C} 1, \mathrm{Ph}), 130.7$ (C2 and C6, Ph), 130.4 (C4, Ph), 126.8 (C3 and C5, Ph), $116.2\left({ }^{1} J_{\mathrm{CF}} 291 \mathrm{~Hz}, \mathrm{C} 3\right.$, propylidene), 92.6 (C1, propylidene), 27.7 (C4, pyrrolidin-2-one), 26.9 (C3, pyrrolidin2-one), 21.1 (4-Me). MS (EI, $70 \mathrm{eV}): \mathrm{m} / z$ (\%): $283\left(\mathrm{M}^{+}\right.$, 24), 214 (100), 186 (53), 158 (17), 91 (43).

5f: Yield $91 \%$; mp 88-90 ${ }^{\circ} \mathrm{C}$; Anal. Calcd. for $\mathrm{C}_{14} \mathrm{H}_{12} \mathrm{~F}_{3} \mathrm{NO}_{3}\left(299.25 \mathrm{~g} \cdot \mathrm{mol}^{-1}\right)$ : C, 56.19; H, 4.04, N, 4.68 . Found: C, 55.91; H, 4.10; N, 4.51. ${ }^{1} \mathrm{H}$ NMR (400 MHz, $\left.\mathrm{CDCl}_{3}\right) \delta 2.81(\mathrm{~m}, \mathrm{H} 3), 3.50(\mathrm{~m}, \mathrm{H} 4), 5.66(\mathrm{~s}, \mathrm{CH}), 3.87$ (OMe), $7.05(3 \mathrm{H}, \mathrm{Ph}), 7.12(2 \mathrm{H}, \mathrm{Ph}) .{ }^{13} \mathrm{C}$ NMR (100 MHz, CDCl3) $\delta 178.9\left({ }^{2} J_{\mathrm{CF}} 34 \mathrm{~Hz}, \mathrm{C} 2\right.$, propylidene), $176.6(\mathrm{C} 5$, pyrrolidin-2-one), 169.7 (C2, pyrrolidin-2-one), 160.3 (C4, $\mathrm{Ph}), 128.2$ (C1, Ph), 125.6 (C3 and $\mathrm{C} 5, \mathrm{Ph}), 115.3$ (C2 and $\mathrm{C} 6, \mathrm{Ph}), 116.3\left({ }^{1} J_{\mathrm{CF}} 291 \mathrm{~Hz}, \mathrm{C} 3\right.$, propylidene), 92.4 (C1, propylidene), 55.4 (OMe), 27.6 (C4, pyrrolidin-2-one), 26.8 (C3, pyrrolidin2-one). MS (EI, $70 \mathrm{eV}): \mathrm{m} / z(\%): 299$ $\left(\mathrm{M}^{+}, 53\right), 230$ (100), 202 (28), 188 (31), 159 (24), 131 (14), 77 (21). 
5g: Yield $70 \%$; mp 163-165 ${ }^{\circ} \mathrm{C}$; Anal. Calcd. for $\mathrm{C}_{13} \mathrm{H}_{9} \mathrm{ClF}_{3} \mathrm{NO}_{2}\left(303.67\right.$ g.mol ${ }^{-1}$ ): C, 56.19; H, 4.04, N, 4.68. Found: C, 55.91; H, 4.10; N, 4.51. ${ }^{1} \mathrm{H}$ NMR (200 $\left.\mathrm{MHz}_{2} \mathrm{CDCl}_{3}\right) \delta 2.82(\mathrm{~m}, \mathrm{H} 3), 3.52(\mathrm{~m}, \mathrm{H} 4), 5.66(\mathrm{~s}, \mathrm{CH})$, $7.16(3 \mathrm{H}, \mathrm{Ph}), 7.54(2 \mathrm{H}, \mathrm{Ph}) .{ }^{13} \mathrm{C} \mathrm{NMR}\left(50 \mathrm{MHz}, \mathrm{CDCl}_{3}\right)$ $\delta 168.7$ (C2), 26.9 (C3), 27.7 (C4), 176.3 (C5), 92.7 (CH) $178.0\left({ }^{2} J_{\mathrm{CF}} 35 \mathrm{~Hz}, \mathrm{C}=\mathrm{O}\right), 116.2\left({ }^{1} J_{\mathrm{CF}} 291 \mathrm{~Hz}, \mathrm{CF}_{3}\right), 128.5$, 130.4, 131.5, $135.8(\mathrm{Ph}) . \mathrm{MS}(\mathrm{EI}, 70 \mathrm{eV}): \mathrm{m} / \mathrm{z}(\%): 303$ $\left(\mathrm{M}^{+}, 23\right), 234$ (100), 206 (62), 178 (21), 143 (23), 111 (28), 75 (41).

5h: Yield $95 \%$; mp $151-153{ }^{\circ} \mathrm{C}$; Anal. Calcd. for $\mathrm{C}_{13} \mathrm{H}_{9} \mathrm{BrF}_{3} \mathrm{NO}_{2}\left(348.12\right.$ g.mol ${ }^{-1}$ ): C, 44.85; H, 2.61, N, 4.02. Found: C, 44.75; H, 2.63; N, 3.95. ${ }^{1} \mathrm{H}$ NMR (200 MHz, $\left.\mathrm{CDCl}_{3}\right) \delta 2.82(\mathrm{~m}, \mathrm{H} 3), 3.52$ (m, H4), $5.66(\mathrm{~s}, \mathrm{CH}), 7.1(3 \mathrm{H}$, $\mathrm{Ph}), 7.7(2 \mathrm{H}, \mathrm{Ph}) .{ }^{13} \mathrm{C} \mathrm{NMR}\left(50 \mathrm{MHz}, \mathrm{CDCl}_{3}\right) \delta 168.5(\mathrm{C} 2)$, 26.9 (C3), 27.8 (C4), 176.2 (C5), $92.9(\mathrm{CH}) 179.2\left({ }^{2} J_{\mathrm{CF}} 35\right.$ $\mathrm{Hz}, \mathrm{C}=\mathrm{O}), 116.2\left({ }^{1} J_{\mathrm{CF}} 291 \mathrm{~Hz}, \mathrm{CF}_{3}\right), 124.0,128.8,132.1$, $133.5(\mathrm{Ph})$. MS (EI, $70 \mathrm{eV}): \mathrm{m} / \mathrm{z}(\%): 348\left(\mathrm{M}^{+}, 29\right), 278$ (100), 250 (29), 222 (14), 199 (37), 143 (39), 76 (41).

5j: Yield $73 \%$; mp 165-167 ${ }^{\circ} \mathrm{C}$; Anal. Calcd. for $\mathrm{C}_{12} \mathrm{H}_{9} \mathrm{~F}_{3} \mathrm{~N}_{2} \mathrm{O}_{2}\left(270.21\right.$ g.mol $\left.{ }^{-1}\right): \mathrm{C}, 53.34 ; \mathrm{H}, 3.36, \mathrm{~N}, 10.37$. Found: C, 53.30; H, 3.50; N, 10.60. ${ }^{1} \mathrm{H}$ NMR (200 MHz, $\left.\mathrm{CDCl}_{3}\right) \delta 2.83$ (m, H3), 3.54 (m, H4), 5.94 (s, CH), 7.36 (1H, Py), 7.46 (1H, Py), 7.95 (1H, Py), 8.69 (1H, Py). ${ }^{13} \mathrm{C}$ NMR (50 MHz, $\mathrm{CDCl}_{3}$ ) $\delta=167.1$ (C2), 26.9 (C3), 28.0 (C4), 176.3 (C5), $93.7(\mathrm{CH}) 179.2\left({ }^{2} J_{\mathrm{CF}} 34 \mathrm{~Hz}, \mathrm{C}=\mathrm{O}\right), 116.2$ $\left({ }^{1} J_{\mathrm{CF}} 292 \mathrm{~Hz}, \mathrm{CF}_{3}\right), 122.5,124.5,139.1,147.2,150.1$ (Py). MS (EI, $70 \mathrm{eV}): m / z$ (\%): 201 (18), 173 (100), 145 (20), 131 (43), 78 (52), 69 (8).

5k: Yield $71 \%$; mp 256-258 ${ }^{\circ} \mathrm{C}$; Anal. Calcd. for $\mathrm{C}_{20} \mathrm{H}_{14} \mathrm{~F}_{6} \mathrm{~N}_{2} \mathrm{O}_{4}\left(460.34\right.$ g.mol $\left.{ }^{-1}\right)$ : C, 52.18; H, 3.07, N, 6.09. Found: C, 52.80; H, 3.10; N, 6.50. ${ }^{1} \mathrm{H}$ NMR (400 MHz, $\left.\mathrm{CDCl}_{3}\right) \delta 2.88$ (m, H3), 3.57 (m, H4), 5.77 (s, CH), 7.4 (Ph). $\left.{ }^{13} \mathrm{C} \mathrm{NMR} \mathrm{(100} \mathrm{MHz,} \mathrm{CDCl}_{3}\right) \delta 179.3\left({ }^{2} J_{\mathrm{CF}} 35 \mathrm{~Hz}\right.$, C2, propylidene), 176.2 (C5, pyrrolidin-2-one), 168.2 (C2, pyrrolidin-2-one), 134.2 (2C, C1, Ph), 129.1 (4C, C2, C3, $\mathrm{Ph}), 116.2\left({ }^{1} J_{\mathrm{CF}} 291 \mathrm{~Hz}, \mathrm{C} 3\right.$, propylidene), 93.0 (C1, propylidene), 27.8 (C4, pyrrolidin-2-one), 26.9 (C3, pyrrolidin2-one). MS (EI, $70 \mathrm{eV}): m / z(\%): 460\left(\mathrm{M}^{+}, 36\right), 391$ (100), 363 (10), 161 (20), 143 (15), 69 (15), 55 (33).

6a: Yield $75 \%$; mp $78-80{ }^{\circ} \mathrm{C}$; Anal. Calcd. for $\mathrm{C}_{10} \mathrm{H}_{12} \mathrm{Cl}_{3} \mathrm{NO}_{2}\left(284.57\right.$ g.mol ${ }^{-1}$ ): C, 42.21; H, 4.25, N, 4.92. Found: C, 42.80; H, 4.50; N, 5.30. ${ }^{1} \mathrm{H}$ NMR (400 MHz, $\left.\mathrm{CDCl}_{3}\right) \delta 2.63$ (m, H3), 3.35 (m, H4), 6.17 (s, CH), 3.6 $\left(\mathrm{CH}_{2}\right), 1.65\left(\mathrm{CH}_{2}\right), 0.95(\mathrm{Me}) .{ }^{13} \mathrm{C} \mathrm{NMR}\left(100 \mathrm{MHz}, \mathrm{CDCl}_{3}\right)$ $\delta 180.2$ (C2, propylidene), 177.0 (C5, pyrrolidin-2-one), 167.2 (C2, pyrrolidin-2-one), 97.4 (C3, propylidene), , 90.3 (C1, propylidene), $42.5\left(\mathrm{NCH}_{2}\right), 27.5(\mathrm{C} 4$, pyrrolidin-2one), 26.3 (C3, pyrrolidin-2-one), $19.9\left(\mathrm{CH}_{2}\right), 11.3(\mathrm{Me})$. MS (EI, $70 \mathrm{eV}): \mathrm{m} / \mathrm{z}(\%): 285\left(\mathrm{M}^{+}+2,10\right), 283\left(\mathrm{M}^{+}, 10\right)$, 220 (22), 166 (100), 124 (73), 68 (61). 6c: Yield $87 \%$; mp 145-148 ${ }^{\circ} \mathrm{C}$; Anal. Calcd. for $\mathrm{C}_{10} \mathrm{H}_{12} \mathrm{Cl}_{3} \mathrm{NO}_{2}$ (346.64 g.mol ${ }^{-1}$ ): C, 51.97; H, 4.07, N, 4.04. Found: C, 51.30; H, 3.80; N, 4.00. ${ }^{1} \mathrm{H}$ NMR (400 MHz, $\left.\mathrm{CDCl}_{3}\right) \delta 2.58$ (m, H3), 3.32 (m, H4), 6.18 (s, CH), 2.9 $\left(\mathrm{CH}_{2}\right), 3.85\left(\mathrm{CH}_{2}\right), 7.1-7.4(\mathrm{Ph}) .{ }^{13} \mathrm{C}$ NMR $(100 \mathrm{MHz}$, $\left.\mathrm{CDCl}_{3}\right) \delta 180.0$ (C2, propylidene), 176.6 (C5, pyrrolidin-2one), 166.8 (C2, pyrrolidin-2-one), 137 (C1, Ph), 128.7 (C3 and $\mathrm{C} 5, \mathrm{Ph}), 128,5(\mathrm{C} 4, \mathrm{Ph}), 126.9(\mathrm{C} 2$ and $\mathrm{C} 6, \mathrm{Ph}), 97.3$ (C3, propylidene), $90.3\left(\mathrm{C} 1\right.$, propylidene), $42.2\left(\mathrm{NCH}_{2}\right)$, $32.7\left(\mathrm{CH}_{2} \mathrm{Ph}\right), 27.4$ (C4, pyrrolidin-2-one), 26.2 (C3, pyrrolidin-2-one). MS (EI, $70 \mathrm{eV}): \mathrm{m} / \mathrm{z}(\%): 347\left(\mathrm{M}^{+}+2,7\right)$, $345\left(\mathrm{M}^{+}, 7\right), 282$ (20), 228 (100), 124 (35), 105 (90).

6d: Yield $93 \%$; mp 158-160 ${ }^{\circ} \mathrm{C}$; Anal. Calcd. for $\mathrm{C}_{13} \mathrm{H}_{9} \mathrm{Cl}_{3} \mathrm{NO}_{2}$ (318.59 g.mol-1 ): C, 49.01; H, 3.16, N, 4.40. Found: C, 50.05; H, 3.20; N, 3.80. ${ }^{1} \mathrm{H}$ NMR (200 MHz, $\left.\mathrm{CDCl}_{3}\right) \delta 2.82$ (m, H3), 3.53 (m, H4), 5.97 (s, CH), 7.2 $(2 \mathrm{H}, \mathrm{Ph}), 7.56(3 \mathrm{H}, \mathrm{Ph}) .{ }^{13} \mathrm{C} \mathrm{NMR}\left(50 \mathrm{MHz}, \mathrm{CDCl}_{3}\right) \delta 180.4$ (C2, propylidene), 176.5 (C5, pyrrolidin-2-one), 167.9 (C2, pyrrolidin-2-one), 133.3 (C1, Ph), 130 (C3 and C5, Ph), 129.6 (C2 and C6, Ph) 127.1 (C4, Ph), 97 (C3, propylidene), 92 (C1, propylidene), 28 (C4, pyrrolidin-2-one), 26.4 (C3, pyrrolidin-2-one). MS (EI, $70 \mathrm{eV}): \mathrm{m} / \mathrm{z}(\%): 319\left(\mathrm{M}^{+}+2\right.$, $<5), 317\left(\mathrm{M}^{+},<5\right), 256$ (5), 254 (10), 202 (15), 200 (100), 172 (30), 77 (20).

6e: Yield $95 \%$; mp 175-178 ${ }^{\circ} \mathrm{C}$; Anal. Calcd. for $\mathrm{C}_{14} \mathrm{H}_{12} \mathrm{Cl}_{3} \mathrm{NO}_{2}\left(332.62\right.$ g.mol $\left.{ }^{-1}\right)$ : C, 50.56; H, 3.64, N, 4.21. Found: C, 50.30; H, 3.50; N, 4.60. ' H NMR (200 MHz, $\left.\mathrm{CDCl}_{3}\right) \delta 2.83(\mathrm{~m}, \mathrm{H} 3), 3.52$ (m, H4), 5.98 (s, CH), 2.43 (4-Me), $7.1(2 \mathrm{H}, \mathrm{Ph}), 7.35$ (3H, Ph). ${ }^{13} \mathrm{C} \mathrm{NMR}(50 \mathrm{MHz}$, $\left.\mathrm{CDCl}_{3}\right) \delta 180.5$ (C2, propylidene), 176.5 (C5, pyrrolidin2-one), 168.2 (C2, pyrrolidin-2-one), 133.3 (C1, Ph), 130.0 (C3 and $\mathrm{C} 5, \mathrm{Ph}), 129.6(\mathrm{C} 4, \mathrm{Ph}), 127.1$ (C2 and C6, Ph), 97.0 (C3, propylidene), 92.0 (C1, propylidene), 28.0 (C4, pyrrolidin-2-one), 26.4 (C3, pyrrolidin-2-one), 21.2 (4-Me). MS (EI, $70 \mathrm{eV}): m / z(\%): 333\left(\mathrm{M}^{+}+2,<5\right), 331\left(\mathrm{M}^{+},<5\right)$, 270 (7), 268 (10), 216 (15), 214 (100).

6f: Yield $95 \%$; mp 169-171 ${ }^{\circ} \mathrm{C}$; Anal. Calcd. for $\mathrm{C}_{14} \mathrm{H}_{12} \mathrm{Cl}_{3} \mathrm{NO}_{3}\left(348.62\right.$ g.mol $\left.{ }^{-1}\right): \mathrm{C}, 48.24 ; \mathrm{H}, 3.47, \mathrm{~N}$, 4.02. Found: C, 48.90; H, 4.0; N, 3.78. ${ }^{1} \mathrm{H}$ NMR (200 $\mathrm{MHz}, \mathrm{CDCl}_{3}$ ) $\delta 2.79$ (m, H3), 3.50 (m, H4), 5.98 (s, CH), 3.86 (4-OMe), 7.05 (2H, Ph), 7.15 (3H, Ph). ${ }^{13} \mathrm{C}$ NMR (50 $\mathrm{MHz}, \mathrm{CDCl}_{3}$ ) $\delta 168.4(\mathrm{C} 2), 26.4(\mathrm{C} 3), 27.9$ (C4), 176.7 (C5), $92.0(\mathrm{CH}) 180.5(\mathrm{C}=\mathrm{O}), 97.1\left(\mathrm{CCl}_{3}\right), 55.5$ (4-Me), 115.3, 125.8, 128.3, $160.2(\mathrm{Ph})$. MS (EI, $70 \mathrm{eV}): \mathrm{m} / \mathrm{z}(\%)$ : $349\left(\mathrm{M}^{+}+2,15\right), 347\left(\mathrm{M}^{+}, 15\right), 284$ (22), 230 (100), 188 (78), 159 (44).

6h: Yield $86 \%$; mp 167-170 ${ }^{\circ} \mathrm{C}$; Anal. Calcd. for $\mathrm{C}_{13} \mathrm{H}_{9} \mathrm{BrCl}_{3} \mathrm{NO}_{2}\left(397.49\right.$ g.mol $\left.{ }^{-1}\right): \mathrm{C}, 39.28 ; \mathrm{H}, 2.28, \mathrm{~N}$, 3.52. Found: C, 39.73; H, 2.5; N, 3.70. ${ }^{1} \mathrm{H}$ NMR (200 $\left.\mathrm{MHz}_{2} \mathrm{CDCl}_{3}\right) \delta 2.83(\mathrm{~m}, \mathrm{H} 3), 3.53(\mathrm{~m}, \mathrm{H} 4), 5.98(\mathrm{~s}, \mathrm{CH})$, 3.86 (4-OMe), 7.10 (2H, Ph), 7.69 (3H, Ph). ${ }^{13} \mathrm{C}$ NMR (50 
$\left.\mathrm{MHz}, \mathrm{CDCl}_{3}\right) \delta 167.2(\mathrm{C} 2), 26.4(\mathrm{C} 3), 27.9(\mathrm{C} 4), 176.2$ (C5), $92.2(\mathrm{CH}) 180.3(\mathrm{C}=\mathrm{O}), 96.9\left(\mathrm{CCl}_{3}\right), 123.6,128.8$, 132.3, $133.3(\mathrm{Ph})$. MS (EI, $70 \mathrm{eV}): \mathrm{m} / \mathrm{z}(\%): 398\left(\mathrm{M}^{+}+2\right.$, < 5), $396\left(\mathrm{M}^{+},<5\right), 280$ (100), 278 (100), 252 (10), 250 (10), 199 (15).

61: Yield $85 \%$; $\mathrm{mp}>300{ }^{\circ} \mathrm{C}$; Anal. Calcd. for $\mathrm{C}_{16} \mathrm{H}_{14} \mathrm{Cl}_{6} \mathrm{~N}_{2} \mathrm{O}_{4}\left(511.02 \mathrm{~g} \cdot \mathrm{mol}^{-1}\right)$ : C, 37.61; H, 2.76, N, 5.48. Found: C, 37.41; H, 2.90; N, 5.70. ${ }^{1} \mathrm{H}$ NMR (400 MHz, $\left.\mathrm{CDCl}_{3}\right) \delta 2.52(\mathrm{~m}, \mathrm{H} 3), 3.17$ (m, H4), $6.22(\mathrm{~s}, \mathrm{CH}), 3.83$ $\left(\mathrm{CH}_{2}\right) .{ }^{13} \mathrm{C} \mathrm{NMR}\left(100 \mathrm{MHz}, \mathrm{CDCl}_{3}\right) \delta 169.1(\mathrm{C} 2), 26.7$ (C3), $28.0(\mathrm{C} 4), 177.6(\mathrm{C} 5), 88.0(\mathrm{CH}) 179.3(\mathrm{C}=\mathrm{O}), 97.1$ $\left(\mathrm{CCl}_{3}\right), 37.2\left(\mathrm{CH}_{2}\right) . \mathrm{MS}(\mathrm{EI}, 70 \mathrm{eV}): \mathrm{m} / z(\%): 359(70), 357$ (100), 236 (20), 234 (35), 150 (32).

\section{Supplementary Information}

Supplementary data shown in Tables $\mathrm{S} 1$ and $\mathrm{S} 2,{ }^{1} \mathrm{H},{ }^{13} \mathrm{C}$ NMR and mass spectra are available free of charge as PDF file at http://jbcs.sbq.org.br

\section{Acknowledgments}

Thanks are due to financial support from Conselho Nacional de Desenvolvimento Científico e Tecnológico (CNPq) and Fundação de Amparo à Pesquisa do Rio Grande do Sul (FAPERGS). The Bruker X-ray diffractometer was funded by infrastructure grant from the Financiadora de Estudos e Projetos (CT-INFRA/FINEP). Students thank CNPq for fellowships.

\section{References}

1. Bonacorso, H. G.; Martins, M. A. P., Bittencourt, S. R. T.; Lourega, R. V.; Zanatta, N.; Flores, A. F. C.; J. Fluorine Chem. 1999, 99, 177 and references cited therein.

2. Druzhinin, S. V.; Balenkova, E. S.; Nenajdenko, V. G.; Tetrahedron 2007, 63, 7753.

3. Martins, M. A. P.; Cunico, W.; Pereira, C. M. P.; Sinhorin, A. P.; Flores, A. F. C.; Bonacorso, H.; Zanatta, N.; Curr. Org. Chem. 2004, 1, 391.

4. Cechin, S. R.; Schetinger, M. R. C.; Zanatta, N.; Madruga, C. C.; Pacholski, I. L.; Flores, D. C.; Bonacorso, H. G.; Martins, M. A. P.; Morsch, V. M. Chem. Res. Toxicol. 2003, 16, 1433.

5. Godoy, M. C.; Fighera, M. R.; Souza, F. R.; Flores, A. E.; Rubin, M. A.; Oliveira, M. R.; Zanatta, N.; Martins, M. A. P.; Bonacorso, H. G.; Mello, C. F.; Eur. J. Pharmacol. 2004, 496, 93.
6. Zanatta, N.; Flores, D. C.; Madruga, C. C.; Flores, A. F. C. ; Bonacorso, H. G.; Martins, M. A. P. Tetrahedron Lett. 2006, 47, 573.

7. Timokhin, B. V.; Baransky, V. A.; Eliseeva, G. D.; Russ. Chem. Rev. 1999, 68, 73.

8. Oliveira, G.; Master Dissertation 2005, Universidade Federal de Santa Maria, Brazil. Precursors $\mathbf{1}$ and $\mathbf{2}$ were identified from mass spectrometric and ${ }^{1} \mathrm{H} /{ }^{13} \mathrm{C}$ NMR data.

9. Shorvon, S.; The Lancet 2001, 358, 1885.

10. Van der Schyf, C. Geldenhuys, W. J.; Youdim, M. B. H.; J. Neurochem. 2006, 99, 1033.

11. Nomura, T.; Nishizaki, T.; Brain Res. 2000, 870, 157.

12. Gorbunova, M. G.; Gerus, I. I.; Kukhar, V. P. Synthesis 2000 , 738, and references therein.

13. Gesser, J. C.; Zucco, C.; Nome, F. J. Phys. Org. Chem. 1995, $8,97$.

14. Vdovenko, S. I.; Gerus, I. I.; Wójcik, J.; J. Phys. Org. Chem. 2001, 14, 533 .

15. Data for methyl 3-(2-methyl-7-(trifluoromethyl)pyrazolo-[1,5a]-pyrimidin-5-yl)propanoate (7) yield $94 \%, \mathrm{mp} 124-125^{\circ} \mathrm{C}$, anal. calcd for $\mathrm{C}_{12} \mathrm{H}_{12} \mathrm{~F}_{3} \mathrm{~N}_{3} \mathrm{O}_{2}: \mathrm{C}, 50.18 ; \mathrm{H}, 4.21 ; \mathrm{N}, 14.63$, found $\mathrm{C}, 50.35 ; \mathrm{H}, 4.02 ; \mathrm{N}, 13.83,{ }^{1} \mathrm{H} \mathrm{NMR}\left(400 \mathrm{MHz}, \mathrm{CDCl}_{3}\right): \delta 2.55$ (s, Me), $2.91\left(\mathrm{t},{ }^{3} J_{\mathrm{HH}} 7.5 \mathrm{~Hz}, \mathrm{CH}_{2}\right), 3.20\left(\mathrm{t},{ }^{3} J_{\mathrm{HH}} 7.5 \mathrm{~Hz}, \mathrm{CH}_{2}\right)$, 3.7 (s, OMe), $6.51(\mathrm{~s}, 1 \mathrm{H}), 6.99(\mathrm{~s}, 1 \mathrm{H}) ;{ }^{13} \mathrm{C}$ NMR $(100 \mathrm{MHz}$, $\left.\mathrm{CDCl}_{3}\right): \delta 14.7(\mathrm{Me}), 31.2\left(\mathrm{CH}_{2}\right), 32.4\left(\mathrm{CH}_{2}\right), 51.8(\mathrm{OMe})$, 96.9 (Pyrim), 105.7 (Pyr), 119.4 (q, $\left.{ }^{1} J_{\mathrm{CF}} 285 \mathrm{~Hz}, \mathrm{CF}_{3}\right), 133.3$ (q, $\left.{ }^{1} J_{\mathrm{CF}} 32 \mathrm{~Hz}, \mathrm{C} 7\right), 156.4$ (C2), 159.1 (C5), 150.5 (C3a) 172.9 (COOR) and 3-(2-methyl-7-(trichloromethyl)pyrazolo-[1,5-a]pyrimidin-5-yl)propanoate (8) yield $84 \%$, mp 191-193 ${ }^{\circ} \mathrm{C}$, anal calcd for $\mathrm{C}_{12} \mathrm{H}_{12} \mathrm{Cl}_{3} \mathrm{~N}_{3} \mathrm{O}_{2}: \mathrm{C}, 42.82 ; \mathrm{H}, 3.59 ; \mathrm{N}, 12.48$, found $\mathrm{C}$, 42.55; H, 4.00; N, 12.20, ${ }^{1} \mathrm{H} \mathrm{NMR}\left(400 \mathrm{MHz}, \mathrm{CDCl}_{3}\right): \delta=2.25$ (s, Me), $2.72\left(\mathrm{t},{ }^{3} J_{\mathrm{HH}} 7.2 \mathrm{~Hz}, \mathrm{CH}_{2}\right), 2.99\left(\mathrm{t},{ }^{3} J_{\mathrm{HH}} 7.2 \mathrm{~Hz}, \mathrm{CH}_{2}\right.$ ), 3.57 (s, OMe), 5.59 (s, 1H), $5.91(\mathrm{~s}, 1 \mathrm{H}) ;{ }^{13} \mathrm{C} \mathrm{NMR}(100 \mathrm{MHz}$, $\left.\mathrm{CDCl}_{3}\right): \delta 14.1(\mathrm{Me}), 28.0\left(\mathrm{CH}_{2}\right), 32.1\left(\mathrm{CH}_{2}\right), 51.9(\mathrm{OMe}), 89.6$ $\left(\mathrm{CCl}_{3}\right), 94.6$ (Pyrim), 102.1(Pyr), 142.2 (C7), 152.8 (C3a), 153.4 (C2), 157.9 (C5), 172.3 (COOR). For experimental details see Martins, M. A. P.; Cunico, W.; Scapin, E.; Emerich, D. J.; Fiss, G. F.; Rosa, F. A.; Zanatta, N.; Bonacorso, H. G.; Flores, A. F. C.; Lett. Org. Chem. 2006, 3, 358.

Received: June 26, 2007 Published on the web: January 31, 2008 


\section{Efficient Synthesis of New 1-[Alkyl(aryl)]-5-(3,3,3-trihalo-2-oxopropylidene) pyrrolidin-2-ones}

Alex F. C. Flores, ${ }^{*}$ Darlene C. Flores, Graciela Oliveira, Lucas Pizzuti,Rubia M. S. da Silva, Marcos A. P. Martins and Helio G. Bonacorso

\section{NUQUIMHE - Departamento de Química, Universidade Federal de Santa Maria 97105900 Santa Maria-RS, Brazil}

${ }^{1} \mathrm{H}$ and ${ }^{13} \mathrm{C}$ NMR spectra were aquired on a Bruker DPX 400 spectrometer $\left({ }^{1} \mathrm{H}\right.$ at $400.13 \mathrm{MHz}$ and ${ }^{13} \mathrm{C}$ at $\left.100.62 \mathrm{MHz}\right) 5 \mathrm{~mm}$ sample tubes, $298-305 \mathrm{~K}$, digital resolution $\pm 0.01 \mathrm{ppm}$, in $\mathrm{CDCl}_{3}$ 0.02-0.05 $\mathrm{g} \mathrm{mL}^{-1}$, using TMS as internal reference.

Table S1. Reaction conditions for isolation of the methyl 4-alkyl[aryl]amino-6-oxo-7,7,7-trifluoro-4-heptenoates (3) in $\mathrm{CH}_{3} \mathrm{CN}$

\begin{tabular}{lccl}
\hline $\mathrm{RNH}_{2}$ & Time $(\mathrm{h})$ & Temperature $\left({ }^{\circ} \mathrm{C}\right)$ & Product $($ ratio, yield $\%)$ \\
\hline benzylamine & 6 & 85 & $\mathbf{3 b}+\mathbf{5 b}(1: 2,85)$ \\
benzylamine & 24 & 25 & $\mathbf{3 b}(87)$ \\
Phenethylamine & 6 & 80 & $\mathbf{3 c}+\mathbf{5 c}(10: 1,83)$ \\
Phenethylamine & 24 & 25 & $\mathbf{3 c}(80)$ \\
Aniline & 6 & 85 & $\mathrm{RNH}_{2}+\mathbf{1}+\mathbf{3 d}(3: 1,25)$ \\
Aniline & 24 & 85 & $\mathbf{3 d}(87)$ \\
4-methoxyaniline & 24 & 30 & $\mathrm{RNH}_{2}+\mathbf{1}+\mathbf{3 f}$ traces \\
4-methoxyaniline & 6 & 85 & $\mathbf{3 f}(79)$ \\
3-amino-5-methylisoxazole & 6 & 30 & $\mathrm{RNH}_{2}+\mathbf{1}+\mathbf{3 h}(1: 10,60)$ \\
3-amino-5-methylisoxazole & 12 & 30 & $\mathbf{3 h}(75)$ \\
3-amino-5-methylisoxazole & 6 & 85 & $\mathbf{3 h}(85)$ \\
\hline
\end{tabular}

${ }^{a}$ Ratio from integration of the vinylic $\mathrm{H}$ and methoxy group signals.

Table S2. Reaction conditions for methyl 4-alkyl[aryl]amino-6-oxo-7,7,7-trichloro-4-heptenoates and 1-Alkyl(aryl)-5-(3,3,3-trifluoro2-oxopropylidene)pyrrolidin-2-one

\begin{tabular}{|c|c|c|c|c|}
\hline $\mathrm{RNH}_{2}$ & Solvent & Time (h) & Temperature $\left({ }^{\circ} \mathrm{C}\right)$ & Product (ratio, yield ${ }^{a} \%$ ) \\
\hline Isopropylamine & $\mathrm{CH}_{2} \mathrm{Cl}_{2}$ & 2 & -5 & 4a (75) \\
\hline Isopropylamine & $\mathrm{CH}_{3} \mathrm{CN}$ & 2 & -5 & $4 \mathbf{a}+\mathbf{6 a}(1: 1,78)$ \\
\hline Phenethylamine & $\mathrm{CH}_{2} \mathrm{Cl}_{2}$ & 4 & 0 & $4 c(86)$ \\
\hline Phenethylamine & $\mathrm{CH}_{3} \mathrm{CN}$ & 4 & 0 & $\mathbf{4 c}+\mathbf{6 c}(3: 2,89)$ \\
\hline Aniline & $\mathrm{CH}_{2} \mathrm{Cl}_{2}$ & 6 & $0-25$ & $\mathrm{RNH}_{2}+\mathbf{2}+\mathbf{4 d}(3: 1,45)$ \\
\hline Aniline & $\mathrm{MeCN}$ & 6 & 25 & 4d (87) \\
\hline Aniline & $\mathrm{MeCN}$ & 6 & 85 & $\mathbf{4 d}+\mathbf{6 d}(1: 2,76)$ \\
\hline 4-methoxyaniline & $\mathrm{CH}_{2} \mathrm{Cl}_{2}$ & 4 & $0-25$ & $\mathrm{RNH}_{2}+\mathbf{2}+\mathbf{4 f}(2: 1,49)$ \\
\hline 4-methoxyaniline & $\mathrm{MeCN}$ & 6 & 25 & 4f $(68)$ \\
\hline 4-bromoaniline & $\mathrm{CH}_{2} \mathrm{Cl}_{2}$ & 4 & $0-25$ & 4h (89) \\
\hline 4-bromoaniline & $\mathrm{MeCN}$ & 6 & 25 & $4 h+6 h(87)$ \\
\hline 4-bromoaniline & $\mathrm{MeCN}$ & 6 & $80-85$ & $\mathbf{6 h}(90)$ \\
\hline Ethylenediamine & $\mathrm{CH}_{2} \mathrm{Cl}_{2}$ & 24 & 25 & $6 \mathbf{l}(86)$ \\
\hline Ethylenediamine & $\mathrm{CH}_{3} \mathrm{CN}$ & 6 & 25 & $61(85)$ \\
\hline
\end{tabular}

${ }^{a}$ Ratio from integration of the vinylic- $\mathrm{H}$ signals.

*e-mail: alexflores@smail.ufsm.br 


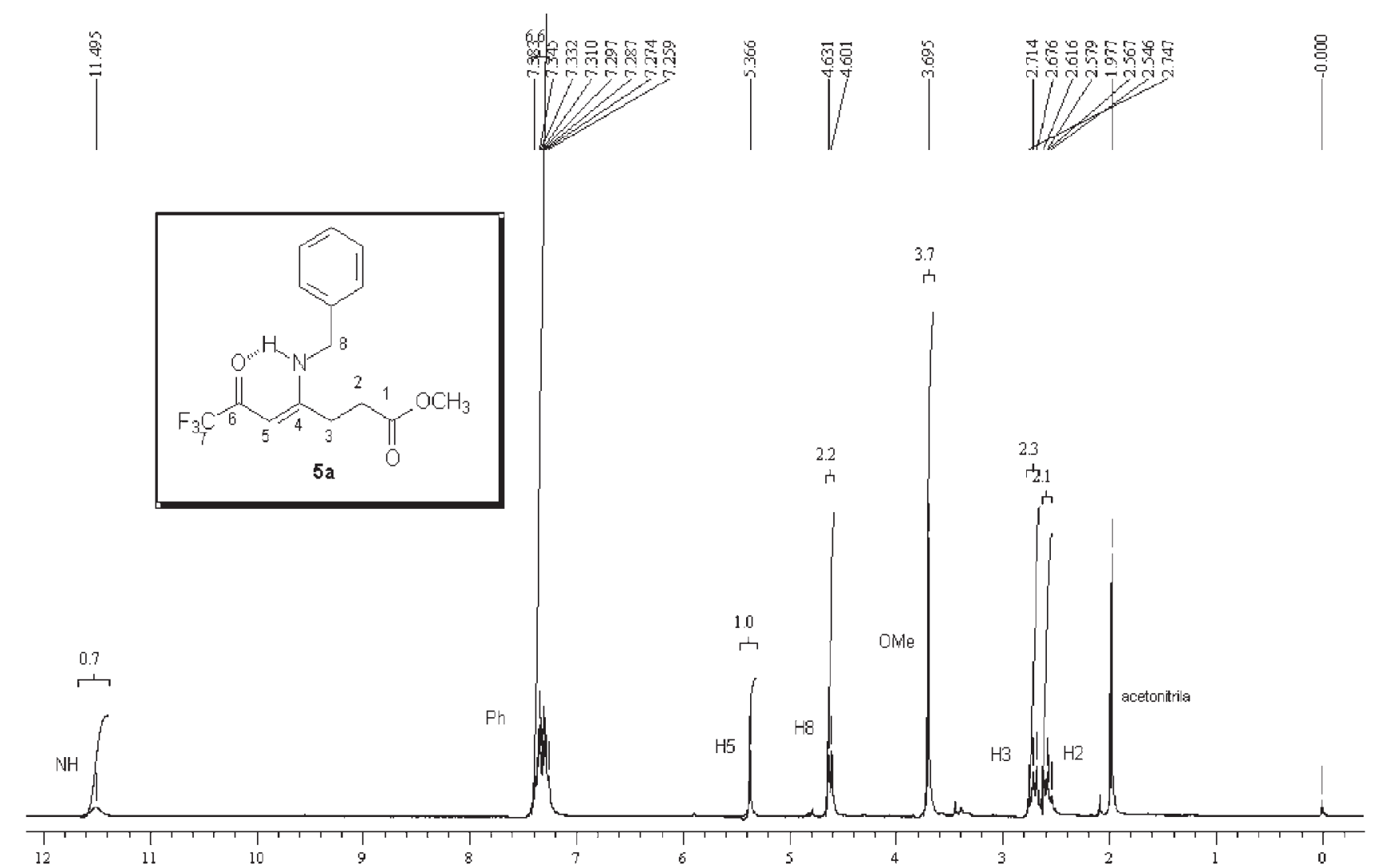

Figure S1. ${ }^{1} \mathrm{H}$ NMR spectrum from methyl 4-benzylamino-6-oxo-7,7,7-trifluoro-4-heptenoate (3b), $400.13 \mathrm{MHz}$ in $\mathrm{CDCl}_{3}$ using TMS as internal standard.
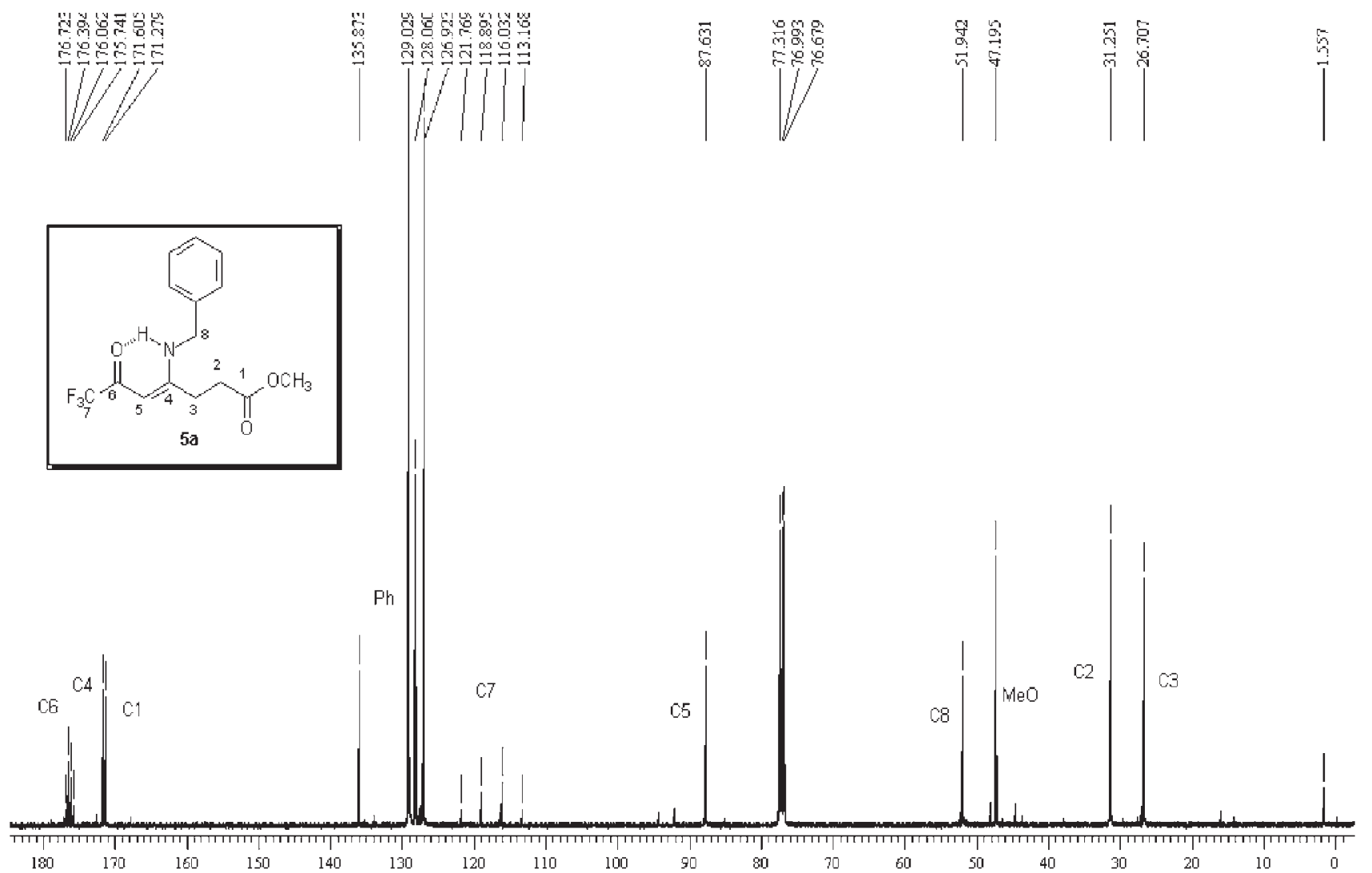
1

2

3

4

5

6

7

8

9

10

11

12

13

14

15

16

17

18

19

20

21

22

23

24

25

26

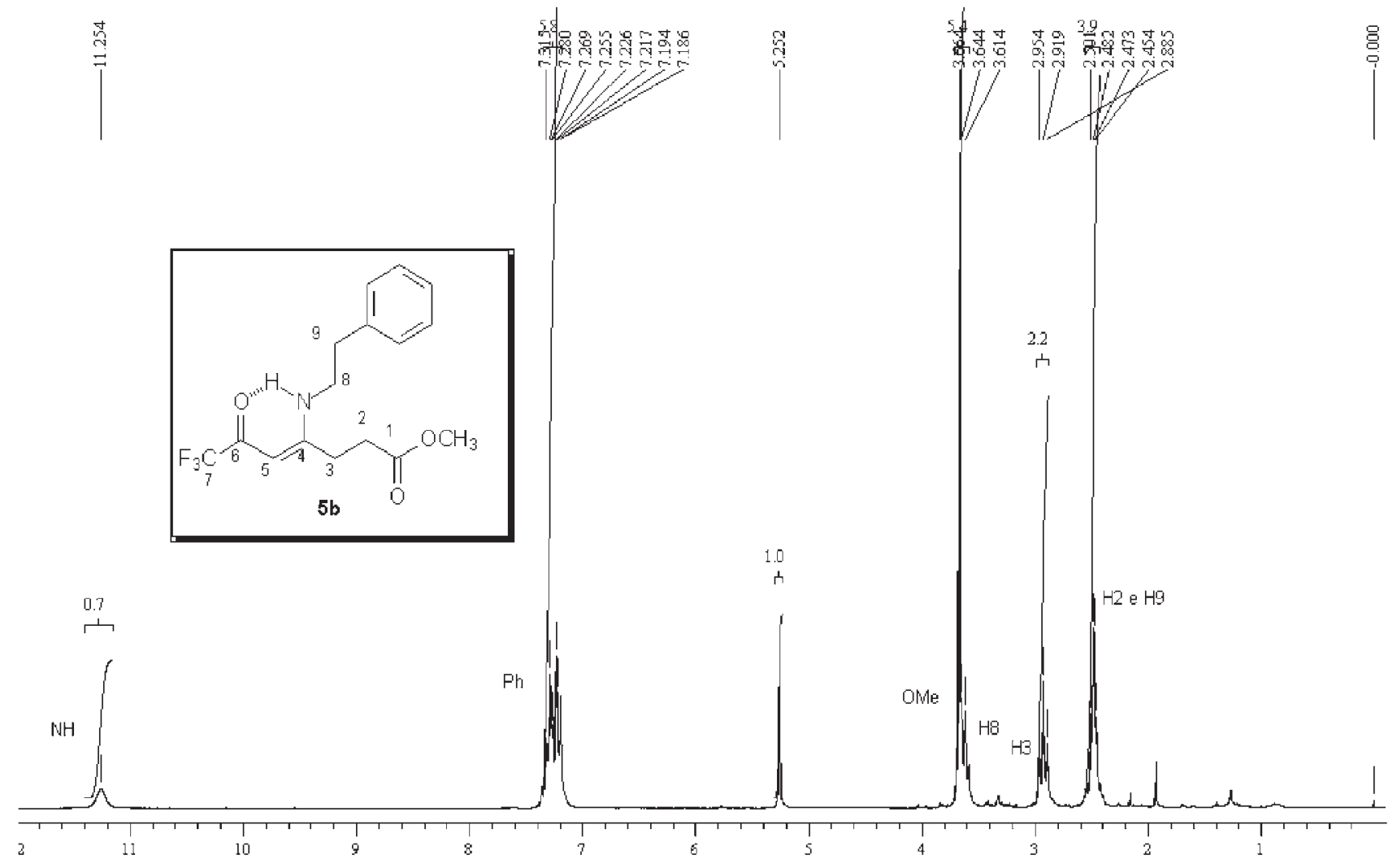

Figure S3. ${ }^{1} \mathrm{H}$ NMR spectrum from methyl 6-oxo-4-(2-phenylethyl)amino-7,7,7-trifluoro-4-heptenoate (3c), $400.13 \mathrm{MHz}$ in $\mathrm{CDCl}_{3}$ using $\mathrm{TMS}_{\text {as internal }}$ standard.

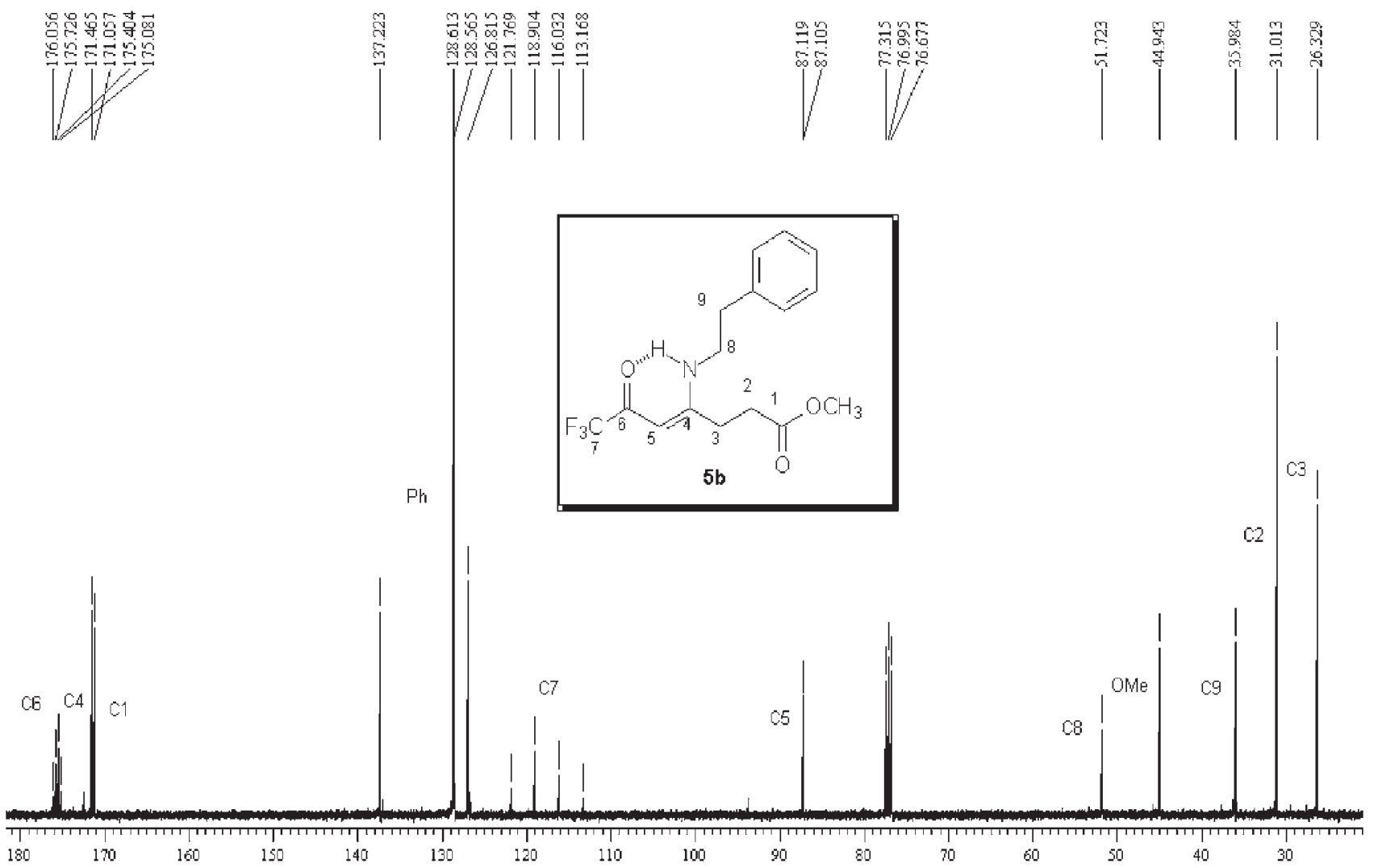

Figure S4. ${ }^{13} \mathrm{C}\{\mathrm{H}\}$ NMR spectrum from methyl 6-oxo-4-(2-phenylethyl)amino-7,7,7-trifluoro-4-heptenoate (3c), 100.62 $\mathrm{MHz}$ in $\mathrm{CDCl}_{3}$ using TMS as internal standard. 


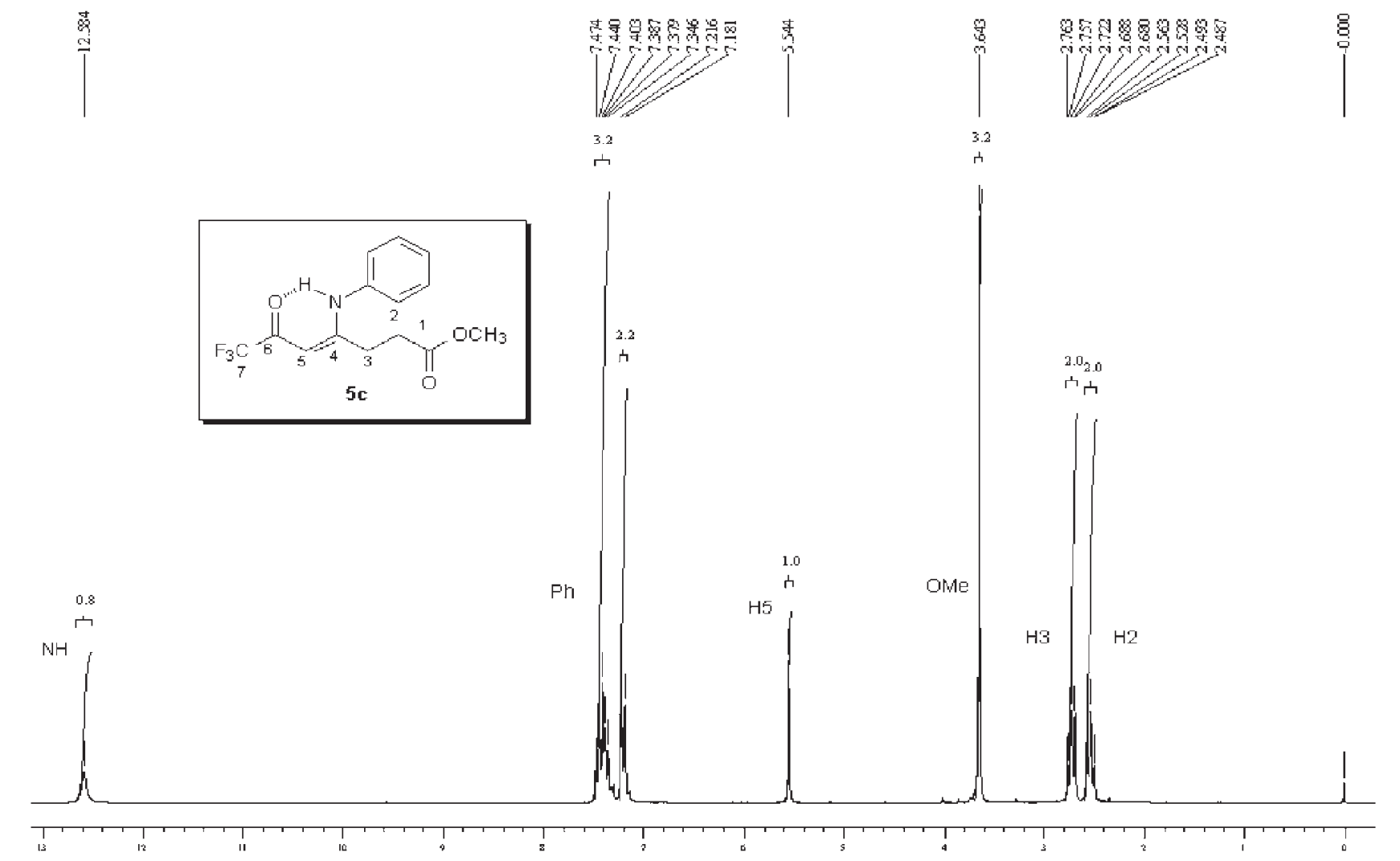

Figure S5. ${ }^{1} \mathrm{H}$ NMR spectrum from methyl 6-oxo-4-phenylamino-7,7,7-trifluoro-4-heptenoate (3d), $400.13 \mathrm{MHz}$ in $\mathrm{CDCl}_{3}$ using TMS as internal standard.
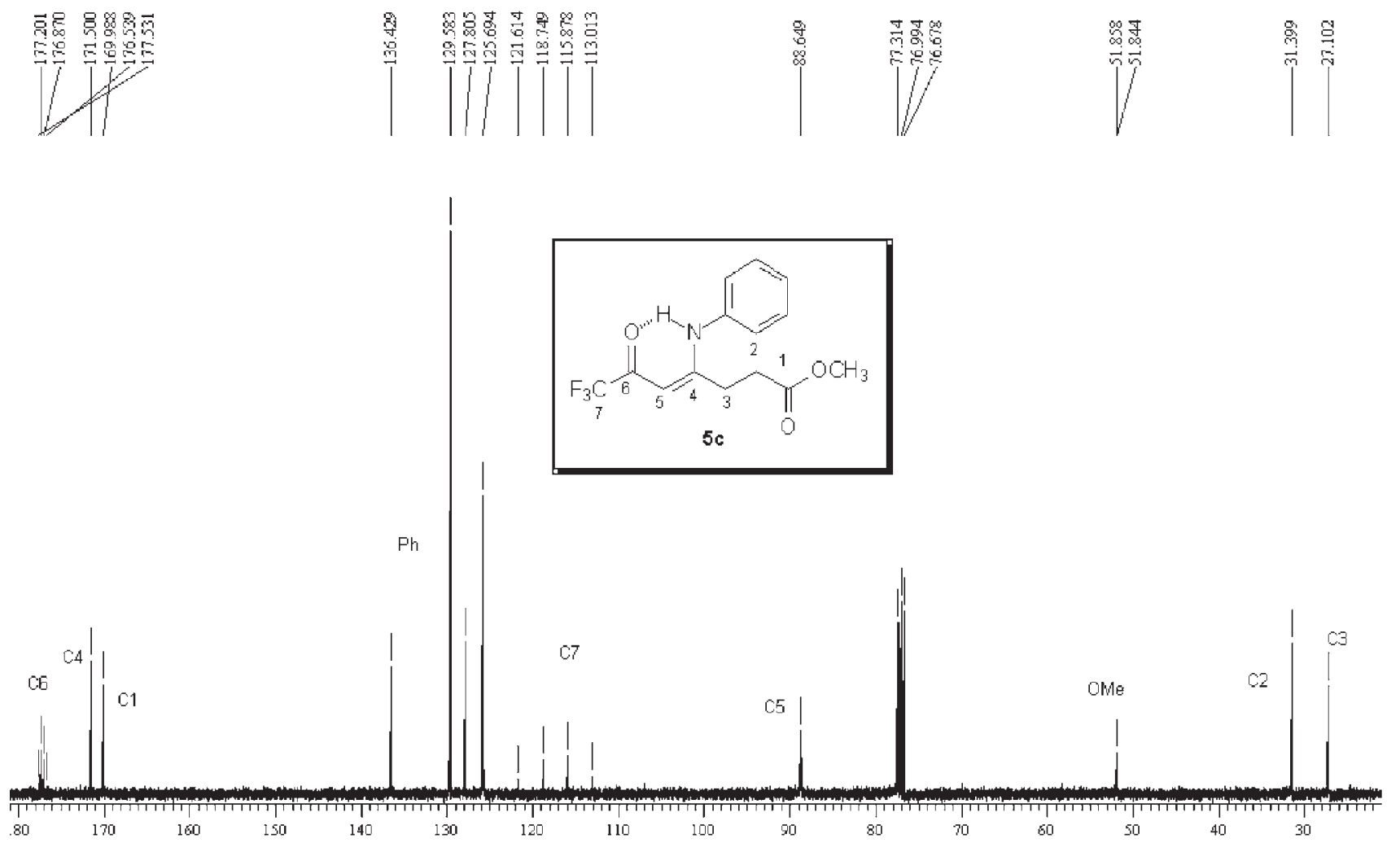

Figure S6. ${ }^{13} \mathrm{C}\{\mathrm{H}\}$ NMR spectrum from methyl 6-oxo-4-phenylamino-7,7,7-trifluoro-4-heptenoate (3d), $100.62 \mathrm{MHz}$ in $\mathrm{CDCl}_{3}$ using TMS as internal standard. 


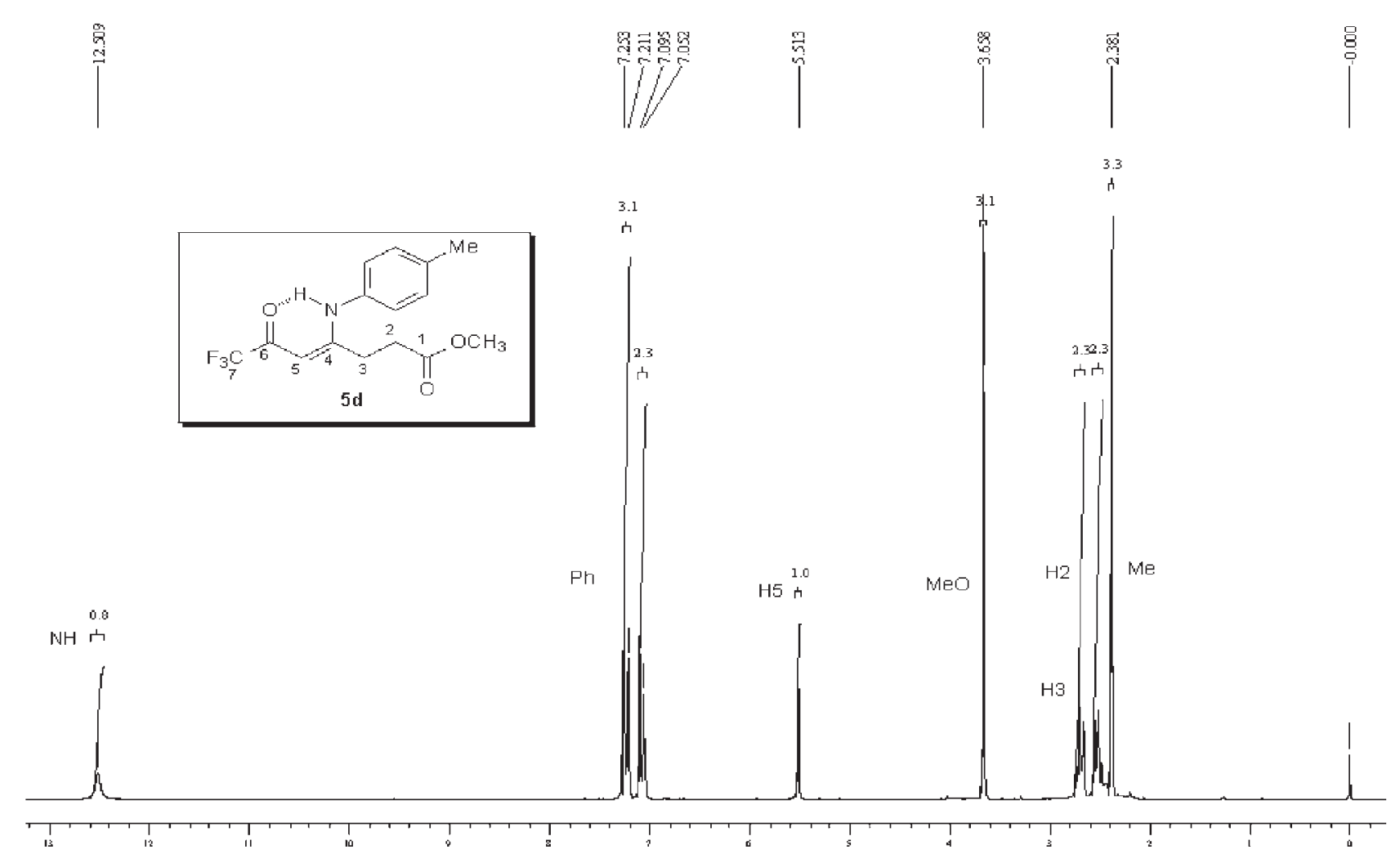

Figurae S7. ${ }^{1} \mathrm{H}$ NMR spectrum from methyl 4-(4-methylphenyl)amino-6-oxo-7,7,7-trifluor-4-heptenoate (3e), 400.13 MHz in CDCl 3 using TMS as internal standard.
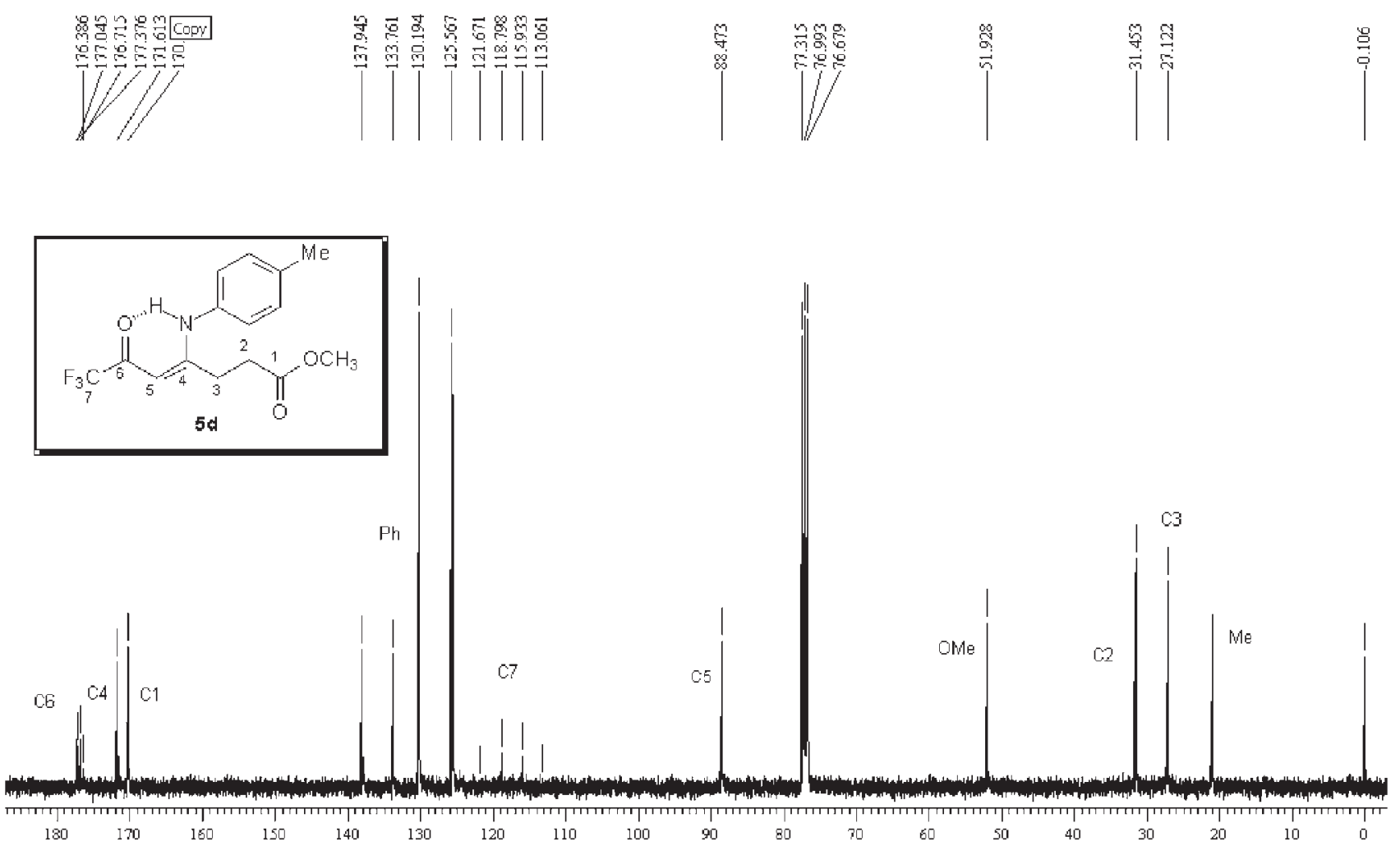

Figure S8. ${ }^{13} \mathrm{C}\{\mathrm{H}\}$ NMR spectrum from methyl 4-(4-methylphenyl)amino-6-oxo-7,7,7-trifluor-4-heptenoate (3e), 100.62 MHz in CDCl ${ }_{3}$ using TMS as internal standard. 


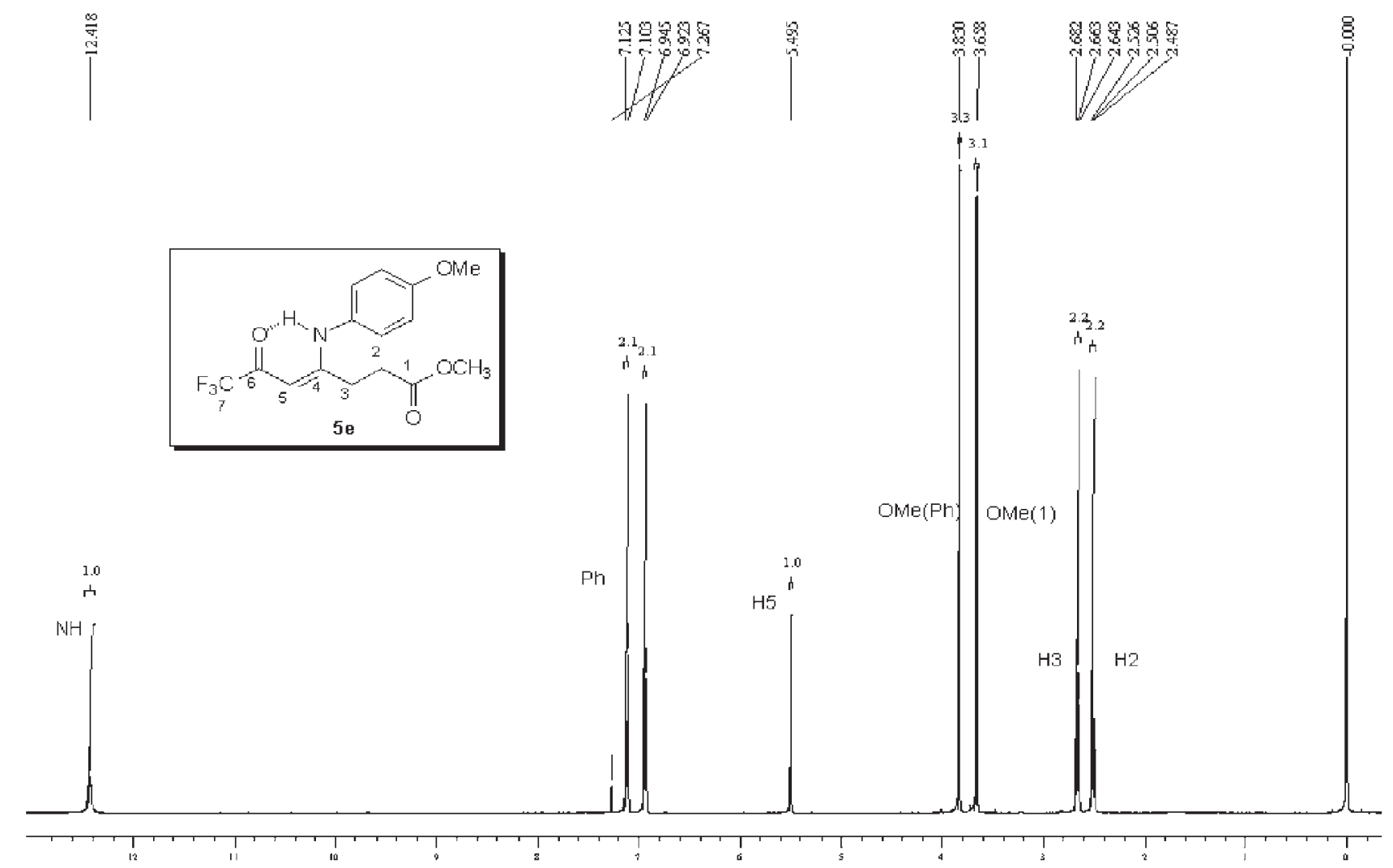

Figure S9. ${ }^{1} \mathrm{H}$ NMR spectrum from methyl 4-(4-methoxyphenyl)amino-6-oxo-7,7,7-trifluor-4-heptenoate (3f), $400.13 \mathrm{MHz}$ in $\mathrm{CDCl}_{3}$ using TMS as internal standard.
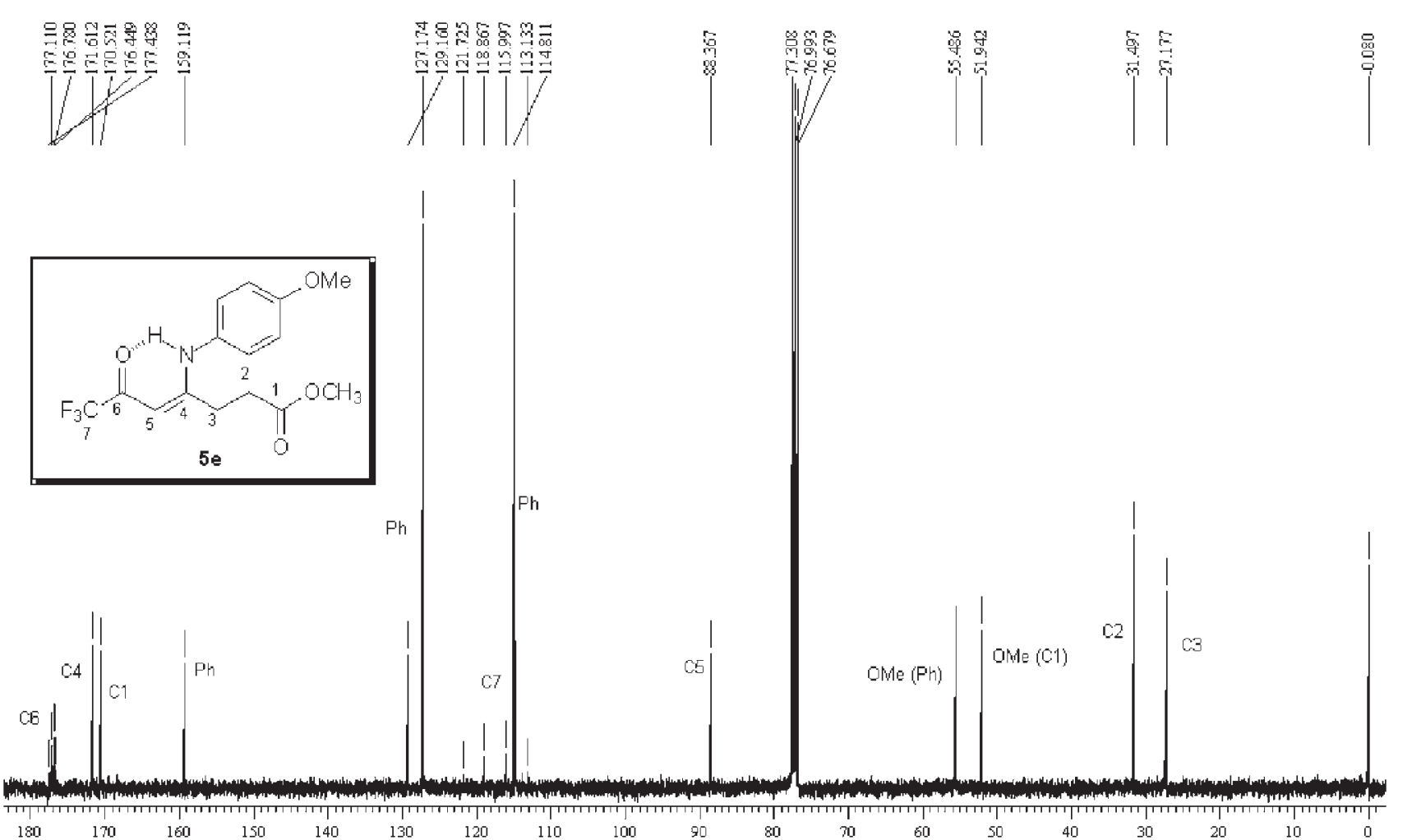

Figure S10. ${ }^{13} \mathrm{C}\{\mathrm{H}\}$ NMR spectrum from methyl 4-(4-methoxyphenyl)amino-6-oxo-7,7,7-trifluor-4-heptenoate (3f), $100.62 \mathrm{MHz}$ in $\mathrm{CDCl}_{3}$ using TMS as internal standard. 


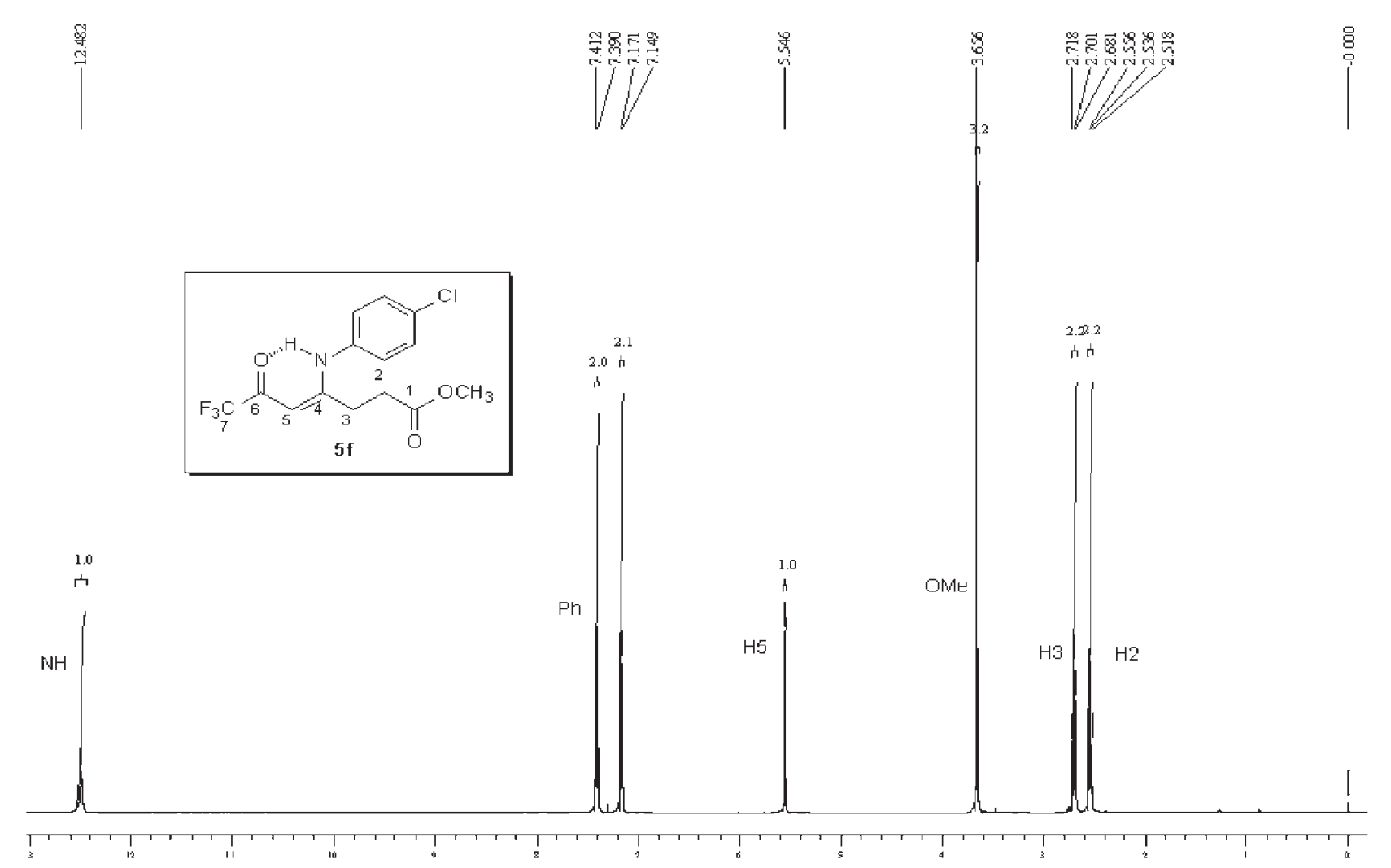

Figure S11. ${ }^{1} \mathrm{H}$ NMR spectrum from methyl 4-(4-chlorophenyl)amino-6-oxo-7,7,7-trifluoro-4-heptenoate (3g), $400.13 \mathrm{MHz}$ in $\mathrm{CDCl}_{3}$ using TMS as internal standard.
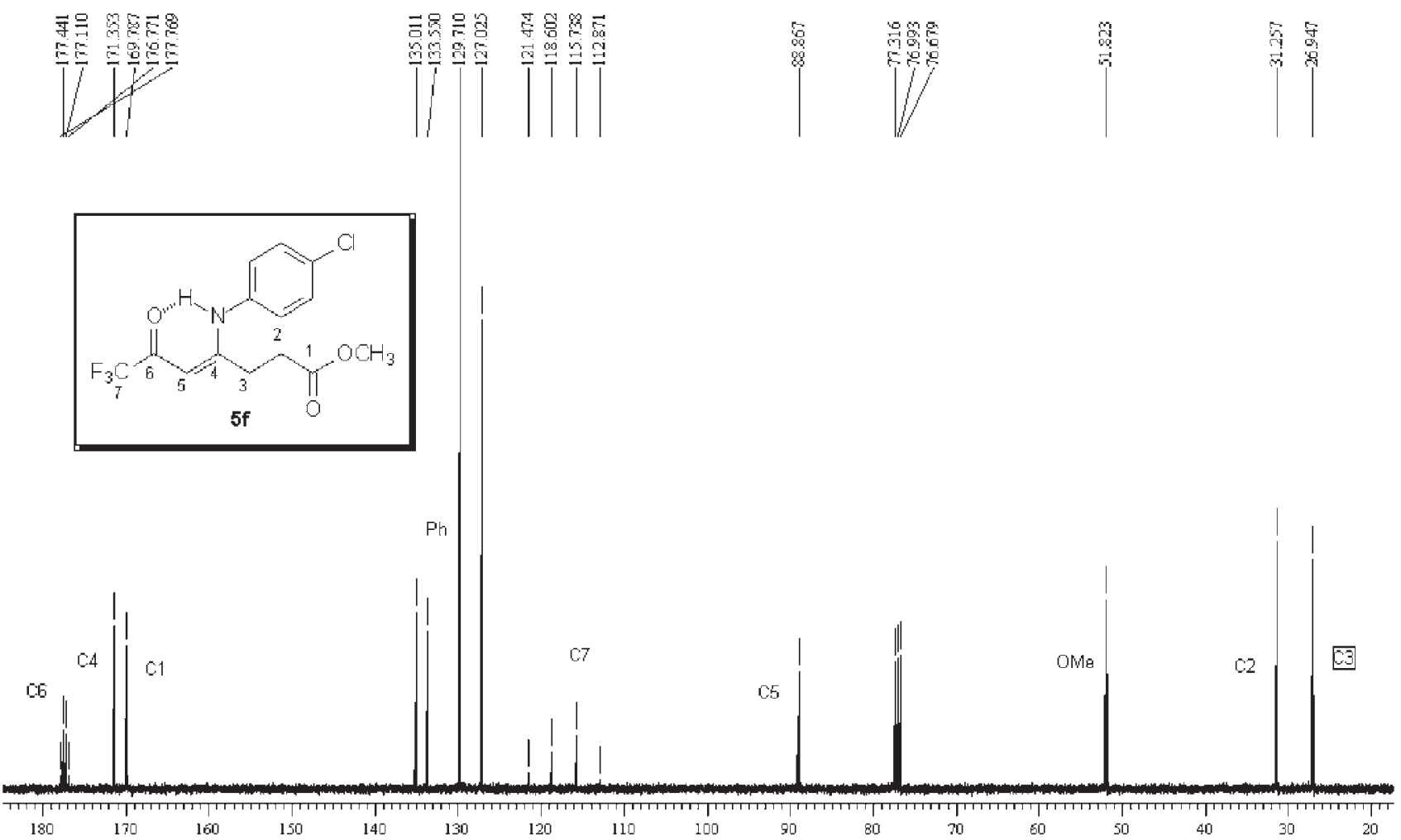

Figure S12. ${ }^{13} \mathrm{C}\{\mathrm{H}\}$ NMR spectrum from methyl 4-(4-chlorophenyl)amino-6-oxo-7,7,7-trifluoro-4-heptenoate (3g), $100.62 \mathrm{MHz}$ in $\mathrm{CDCl}_{3}$ using TMS as internal standard. 


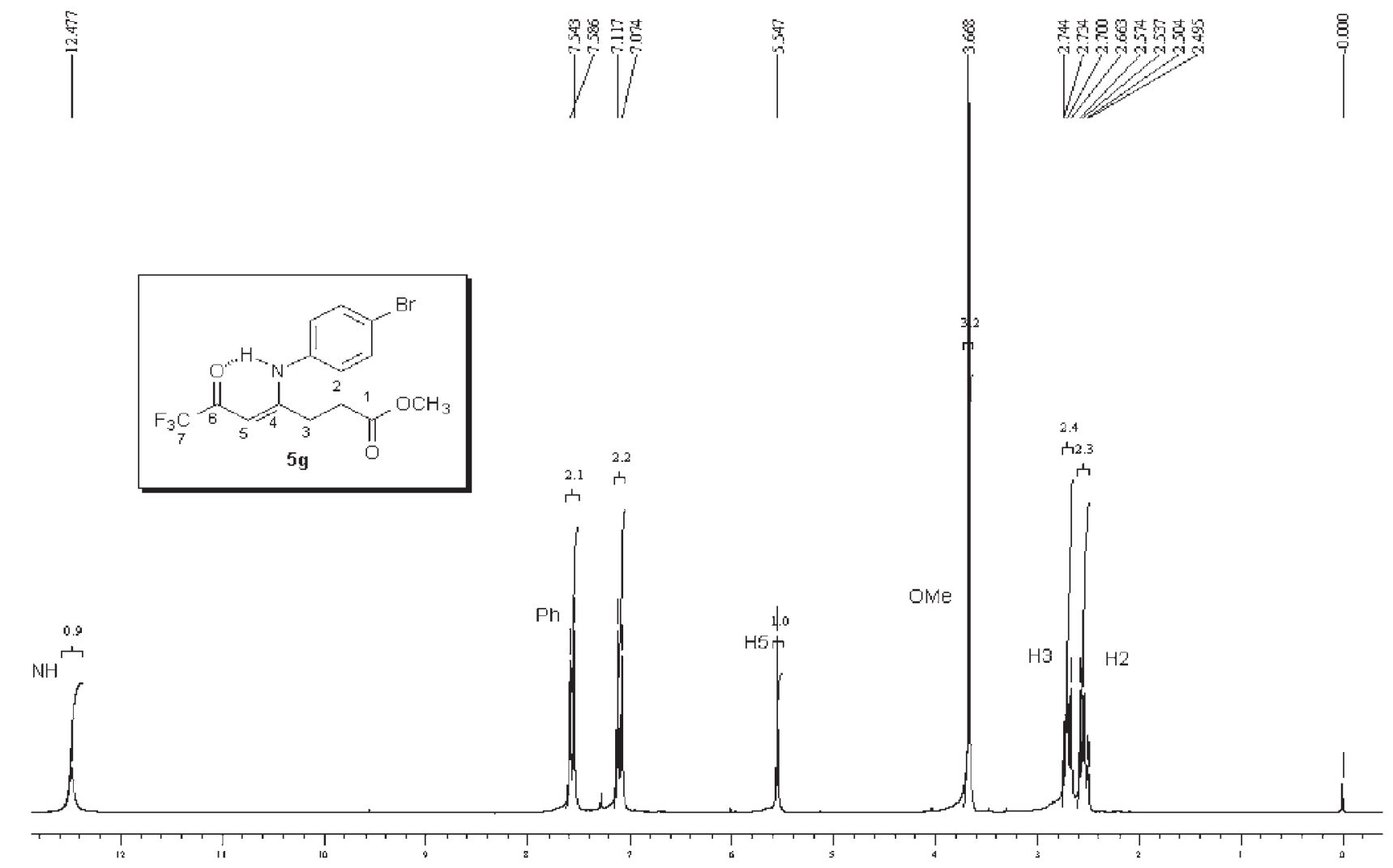

Figure S13. ${ }^{1} \mathrm{H}$ NMR spectrum from 4-(4-bromophenyl)amino-6-oxo-7,7,7-trifluoro-4-heptenoate (3h), $400.13 \mathrm{MHz}$ in $\mathrm{CDCl}_{3}$ using TMS as internal standard.
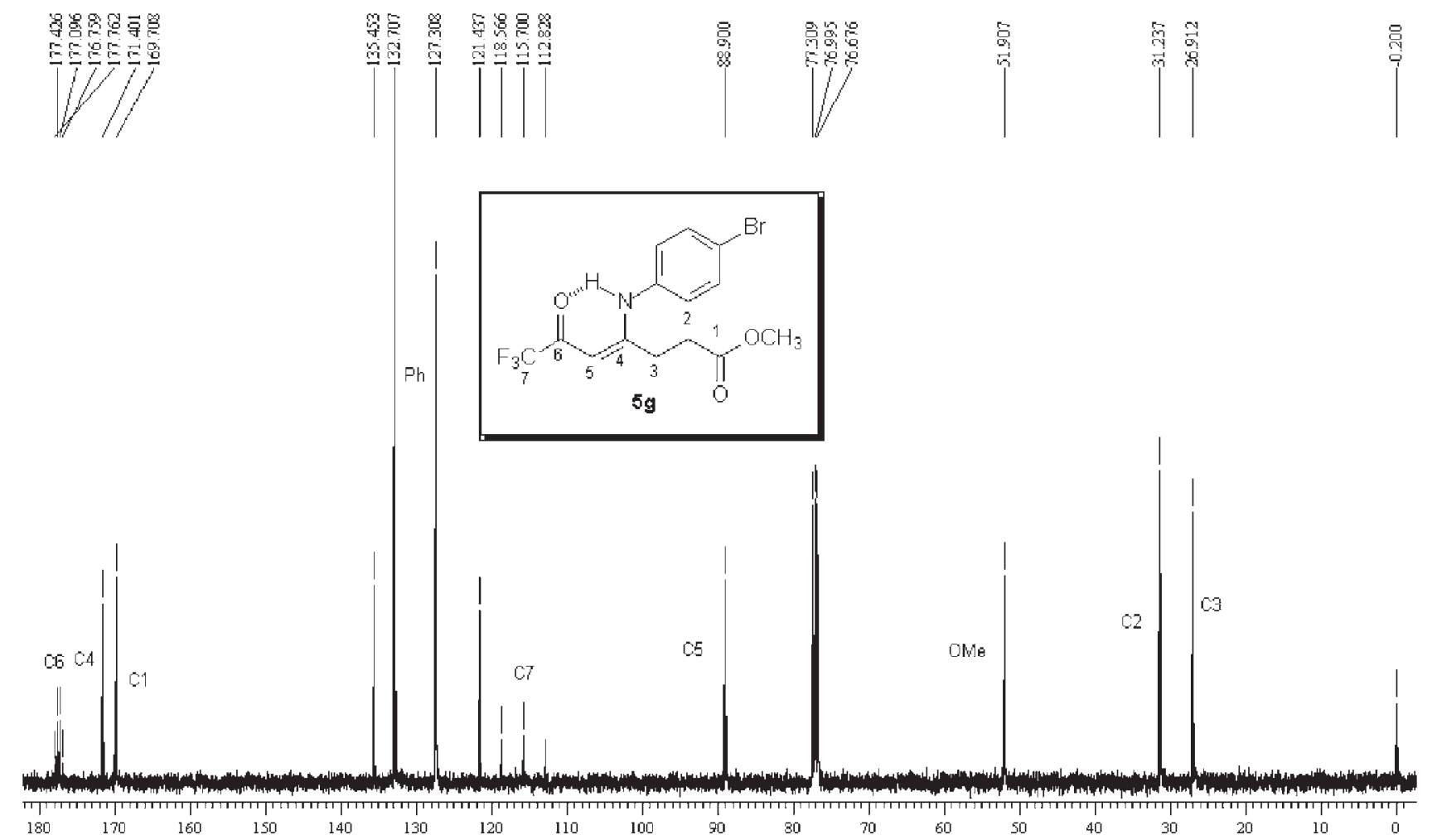

Figure S14. ${ }^{13} \mathrm{C}\{\mathrm{H}\}$ NMR spectrum from 4-(4-bromophenyl)amino-6-oxo-7,7,7-trifluoro-4-heptenoate (3h), $100.62 \mathrm{MHz}$ in $\mathrm{CDCl}$ using TMS as internal standard. 


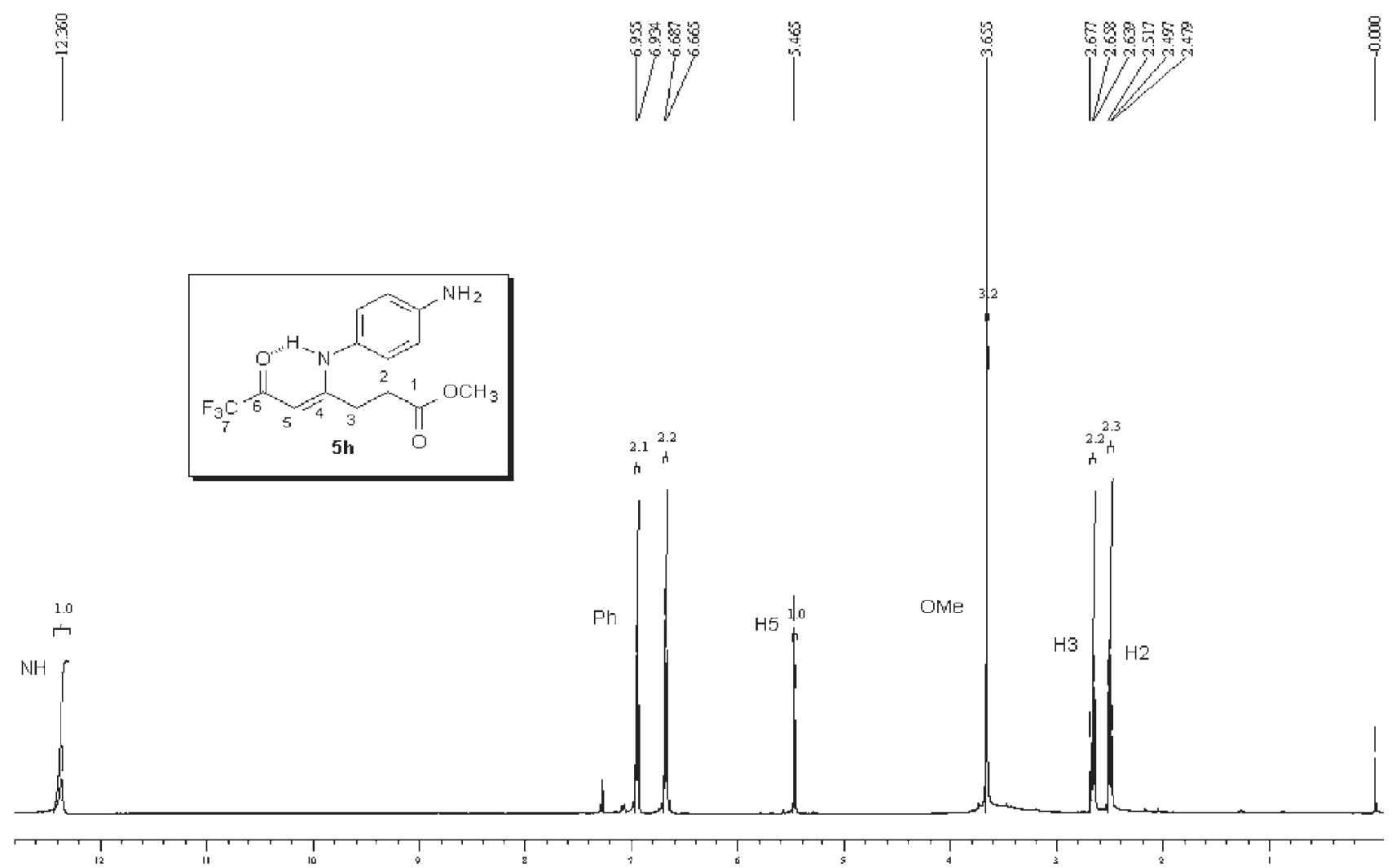

Figure S15. ${ }^{1} \mathrm{H}$ NMR spectrum from methyl 4-(4-aminophenyl)amino-6-oxo-7,7,7-trifluoro-4-heptenoate (3k), 400.13 $\mathrm{MHz}^{\text {in }} \mathrm{CDCl} \mathrm{3}_{3}$ using TMS as internal standard.

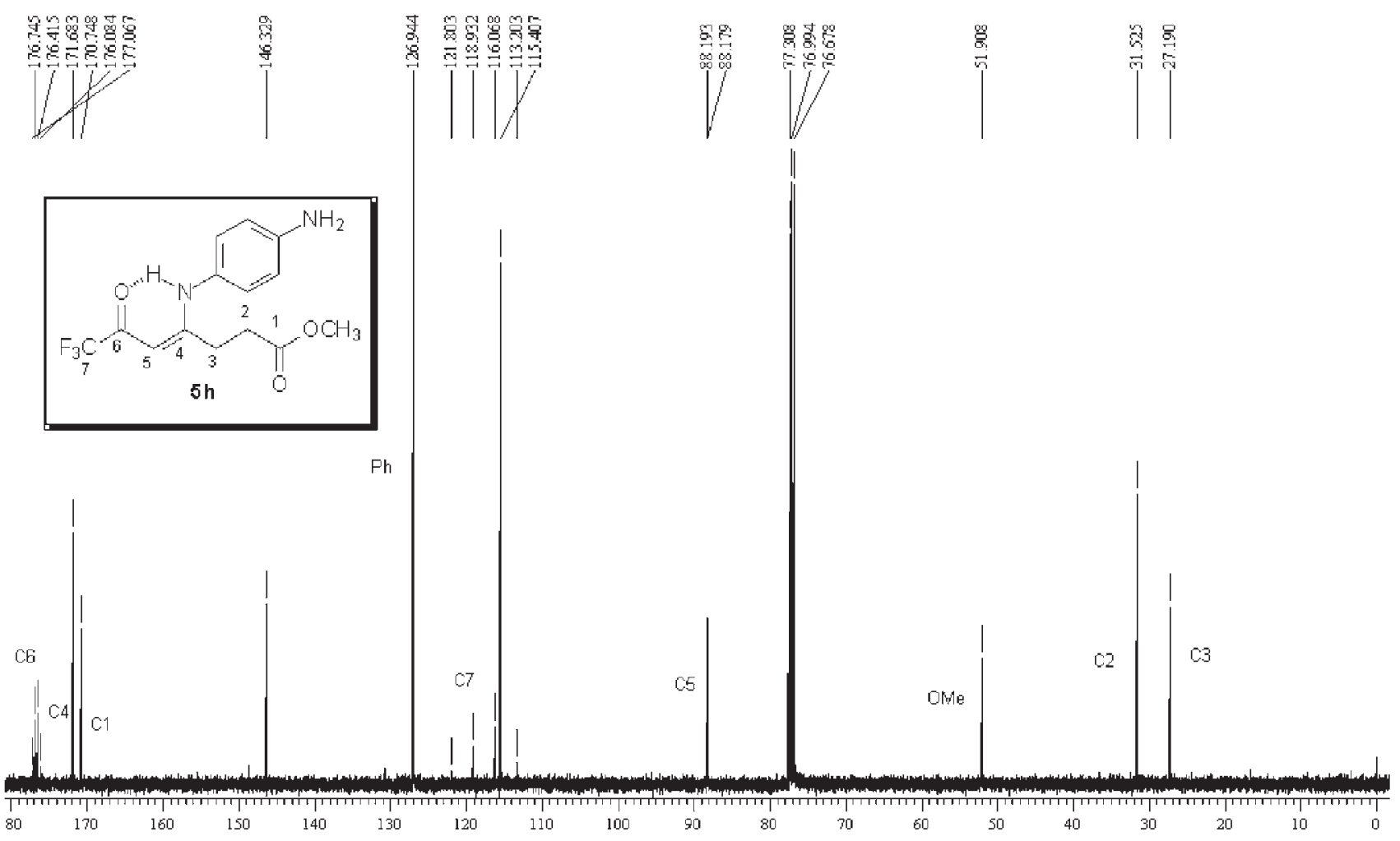

Figure S16. ${ }^{13} \mathrm{C}\{\mathrm{H}\}$ spectrum from methyl 4-(4-aminophenyl)-6-oxo-7,7,7-trifluoro-4-heptenoate (3k), $100.62 \mathrm{MHz}$ in $\mathrm{CDCl}{ }_{3}$ using TMS as internal standard. 


$$
\left.\right|^{\stackrel{9}{8}}
$$
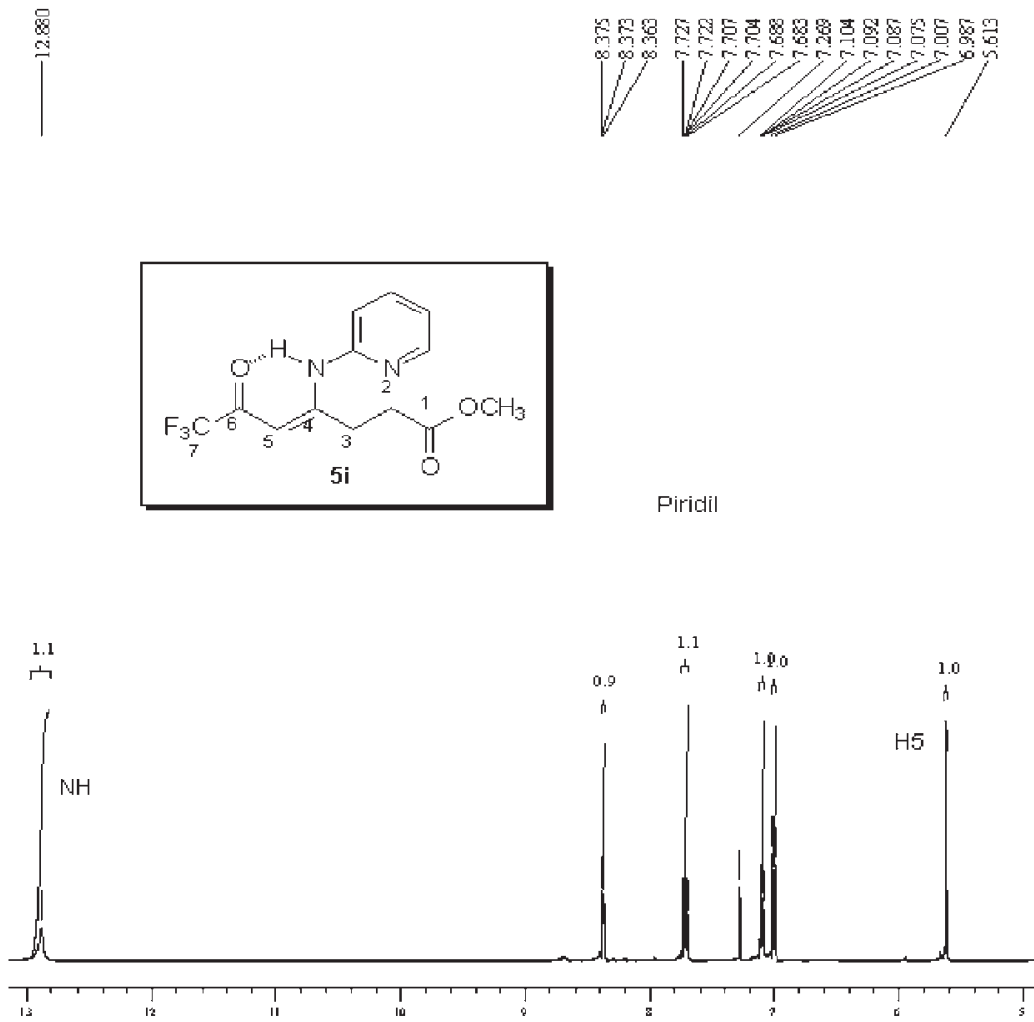

Figure S17. standard.
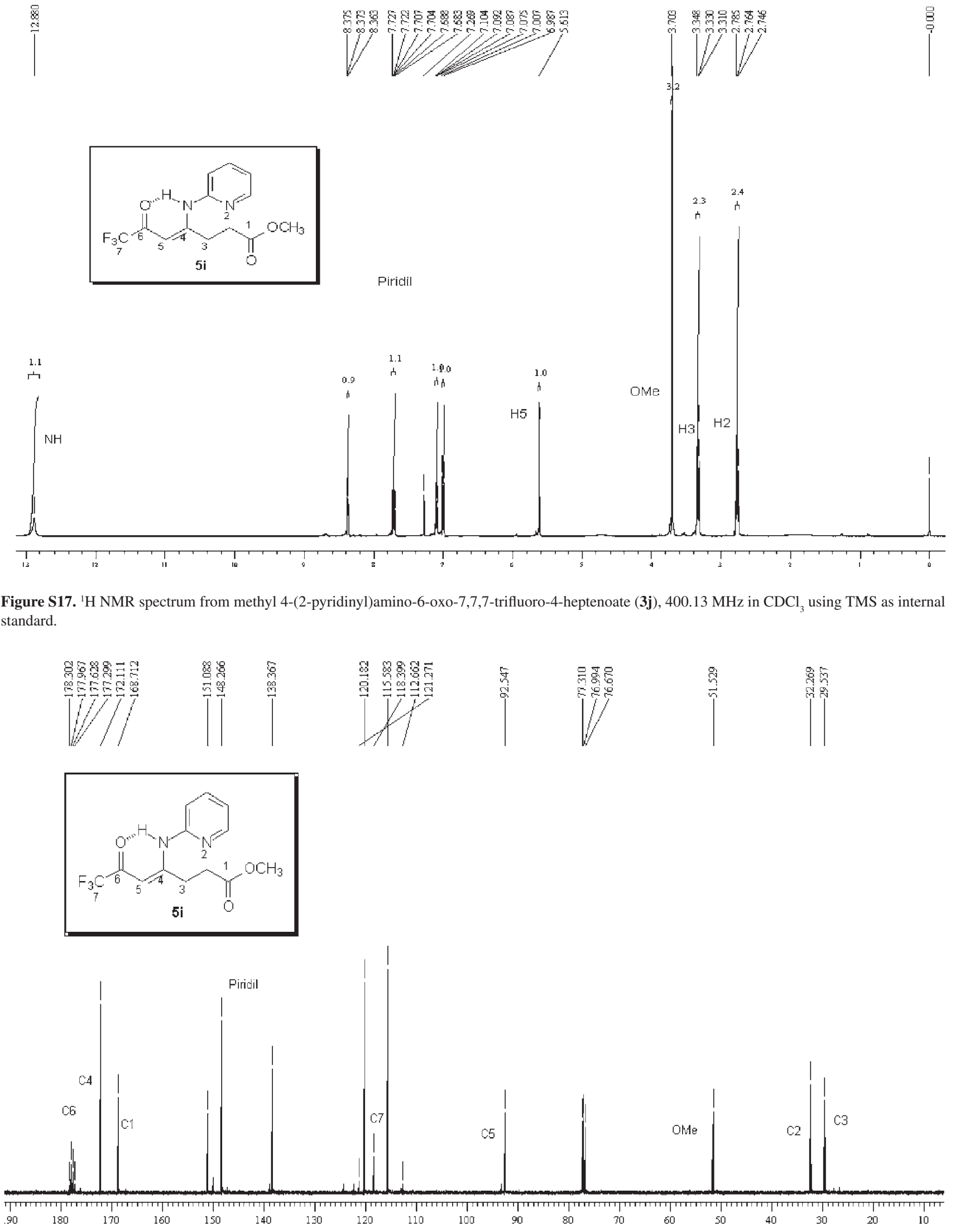

90

Figure S18. ${ }^{13} \mathrm{C}\{\mathrm{H}\}$ NMR spectrum from methyl 4-(2-pyridinyl)amino-6-oxo-7,7,7-trifluoro-4-heptenoate (3j), $100.62 \mathrm{MHz}$ in $\mathrm{CDCl}_{3}$ using TMS as 


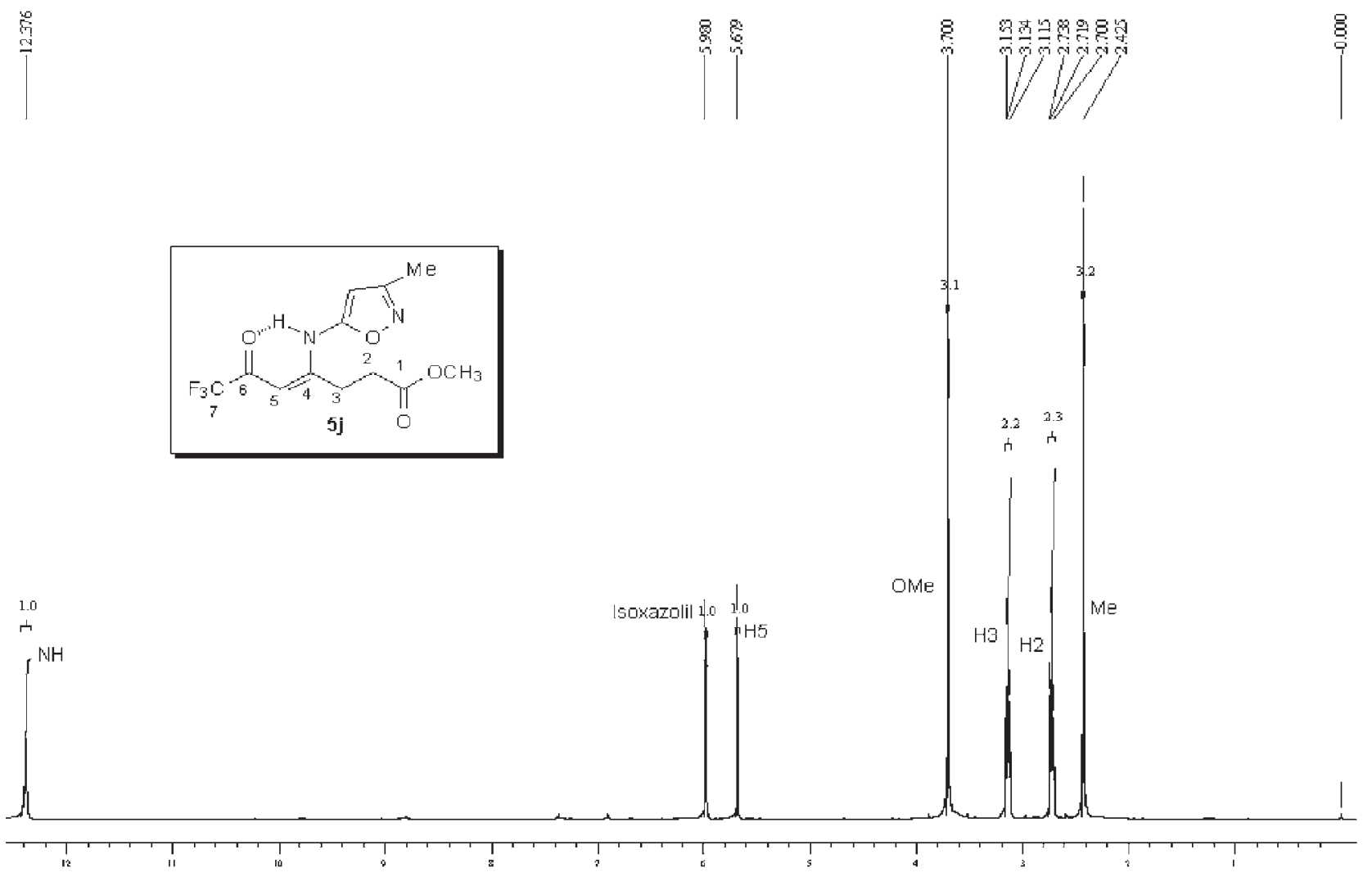

Figure S19. ${ }^{1} \mathrm{H}$ NMR spectrum from methyl 4-(5-methyl-3-isoxazolyl)amino-6-oxo-7,7,7-trifluoro-4-heptenoate (3i), $400.13 \mathrm{MHz}$ in $\mathrm{CDCl}_{3}$ using TMS as internal standard.

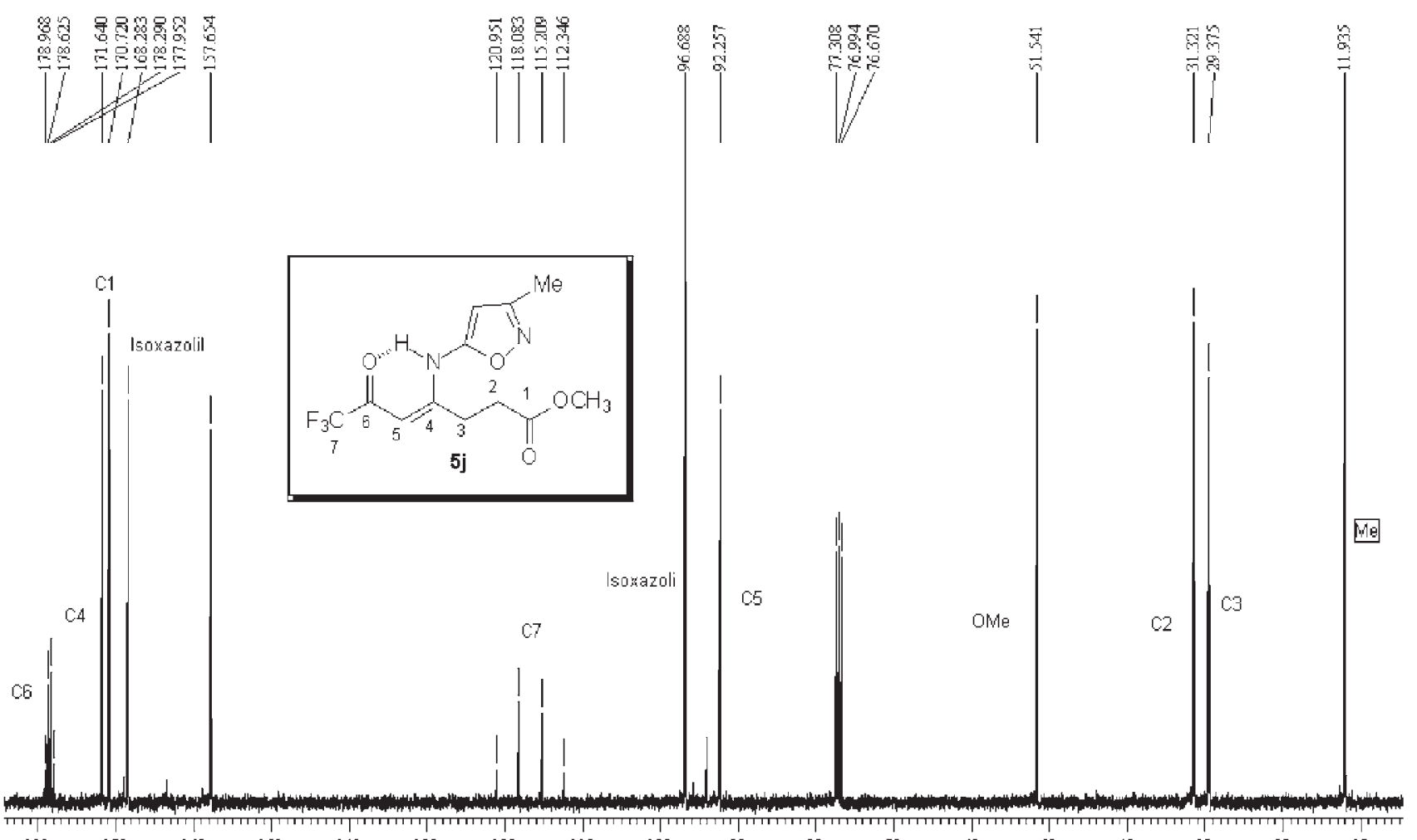

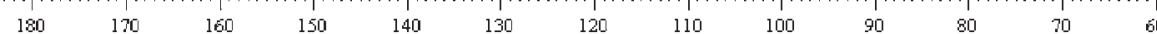

Figure S20. ${ }^{13} \mathrm{C}\{\mathrm{H}\}$ NMR spectrum from methyl 4-(5-methyl-3-isoxazolyl)amino-6-oxo-7,7,7-trifluoro-4-heptenoate (3i), $100.62 \mathrm{MHz}$ in $\mathrm{CDCl}_{3}$ using TMS as internal standard. 


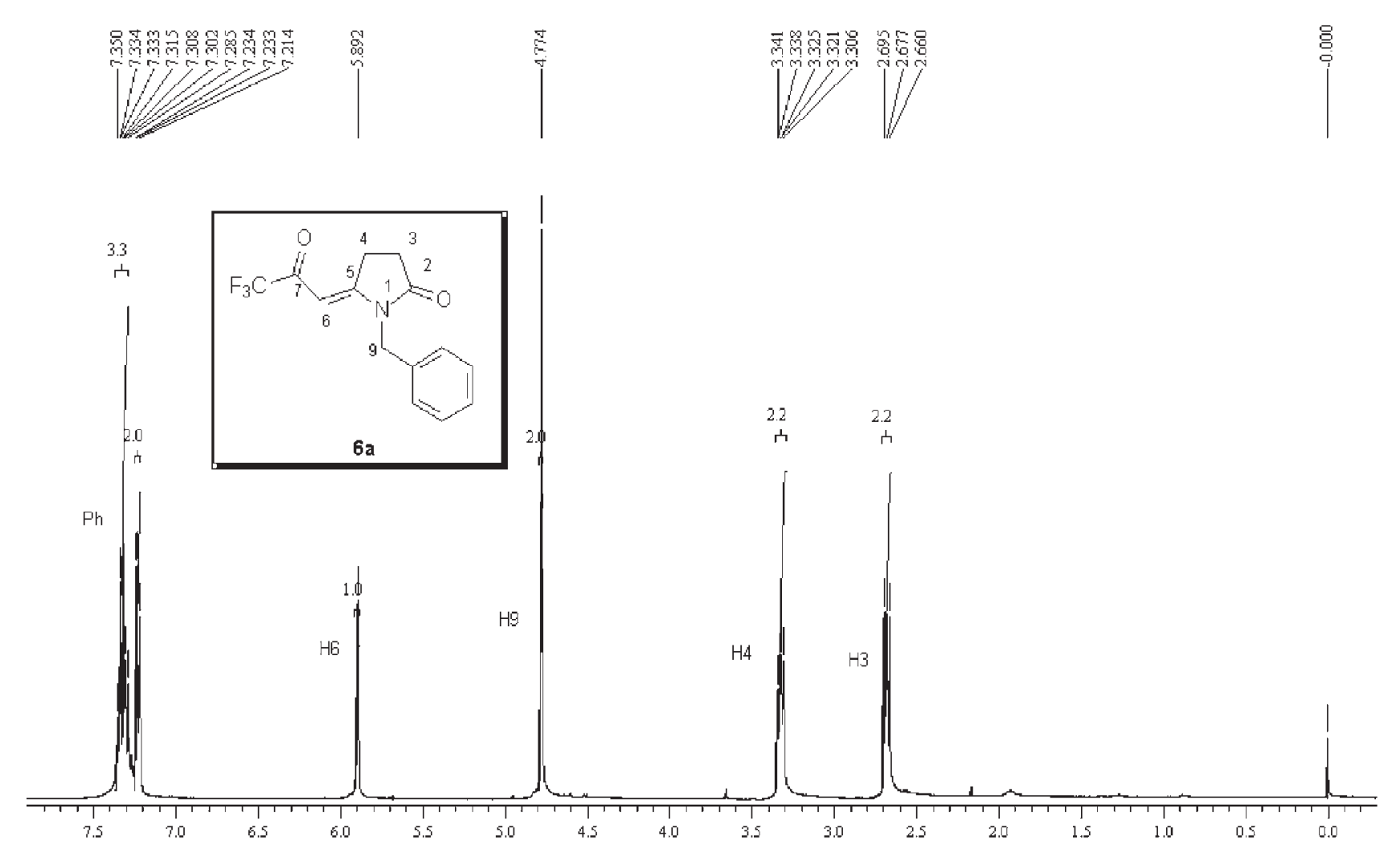

Figure S21. 'H NMR spectrum from 1-benzyl-5-(3,3,3-trifluoro-2-oxopropylidene)pyrrolidin-2-one (5b), $400.13 \mathrm{MHz}$ in $\mathrm{CDCl}_{3}$ using $\mathrm{TMS}_{\text {as }}$ internal standard.
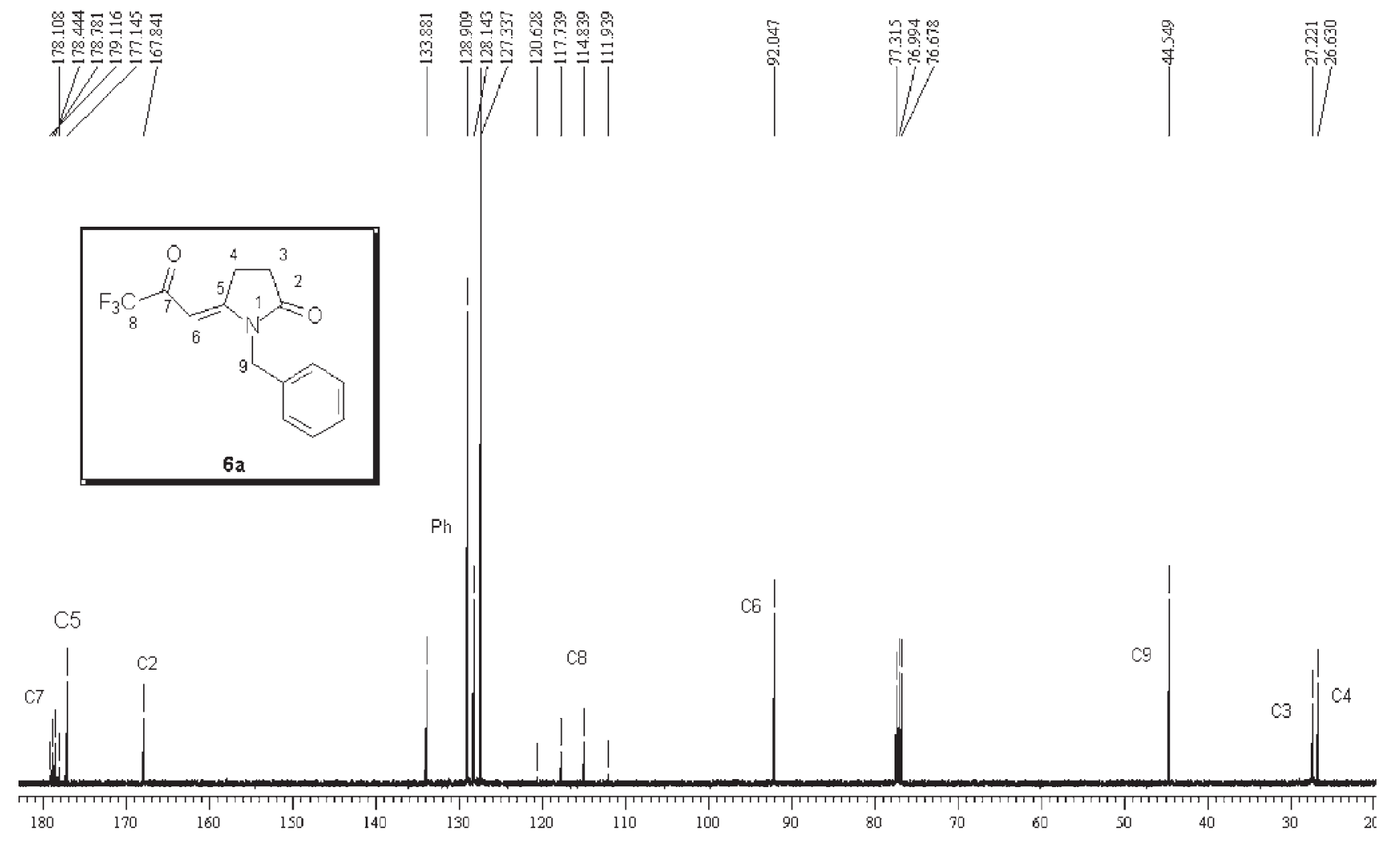

Figure S22. ${ }^{13} \mathrm{C}\{\mathrm{H}\}$ NMR spectrum from 1-benzyl-5-(3,3,3-trifluoro-2-oxopropylidene)pyrrolidin-2-one (5b), $100.62 \mathrm{MHz}$ in $\mathrm{CDCl}_{3}$ using TMS as 


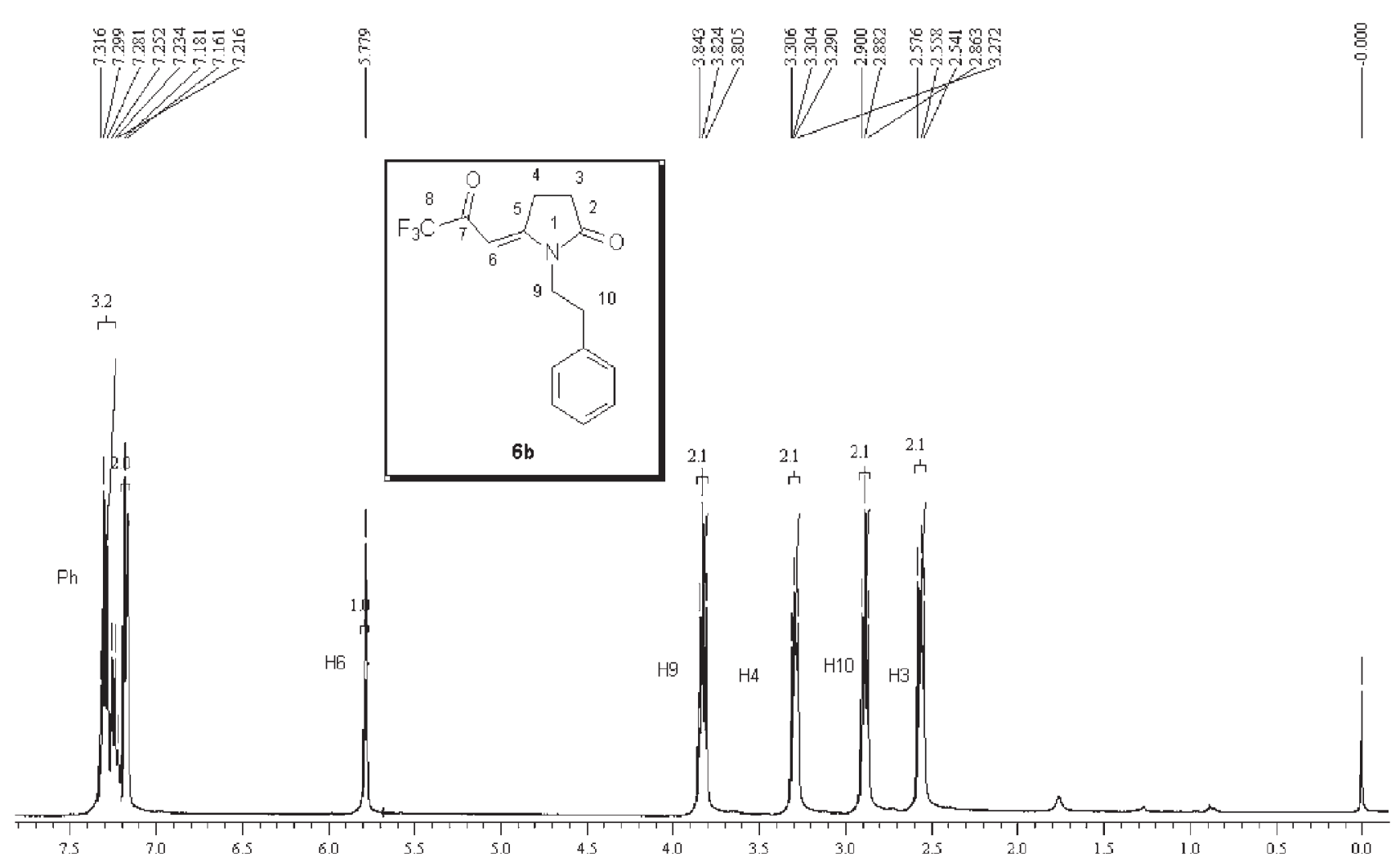

Figure S23. ${ }^{1} \mathrm{H}$ NMR spectrum from 1-(2-phenylethyl)-5-(3,3,3-trifluoro-2-oxopropylidene)pyrrolidin-2-one (5c), $400.13 \mathrm{MHz}$ in $\mathrm{CDCl}_{3}$ using TMS as

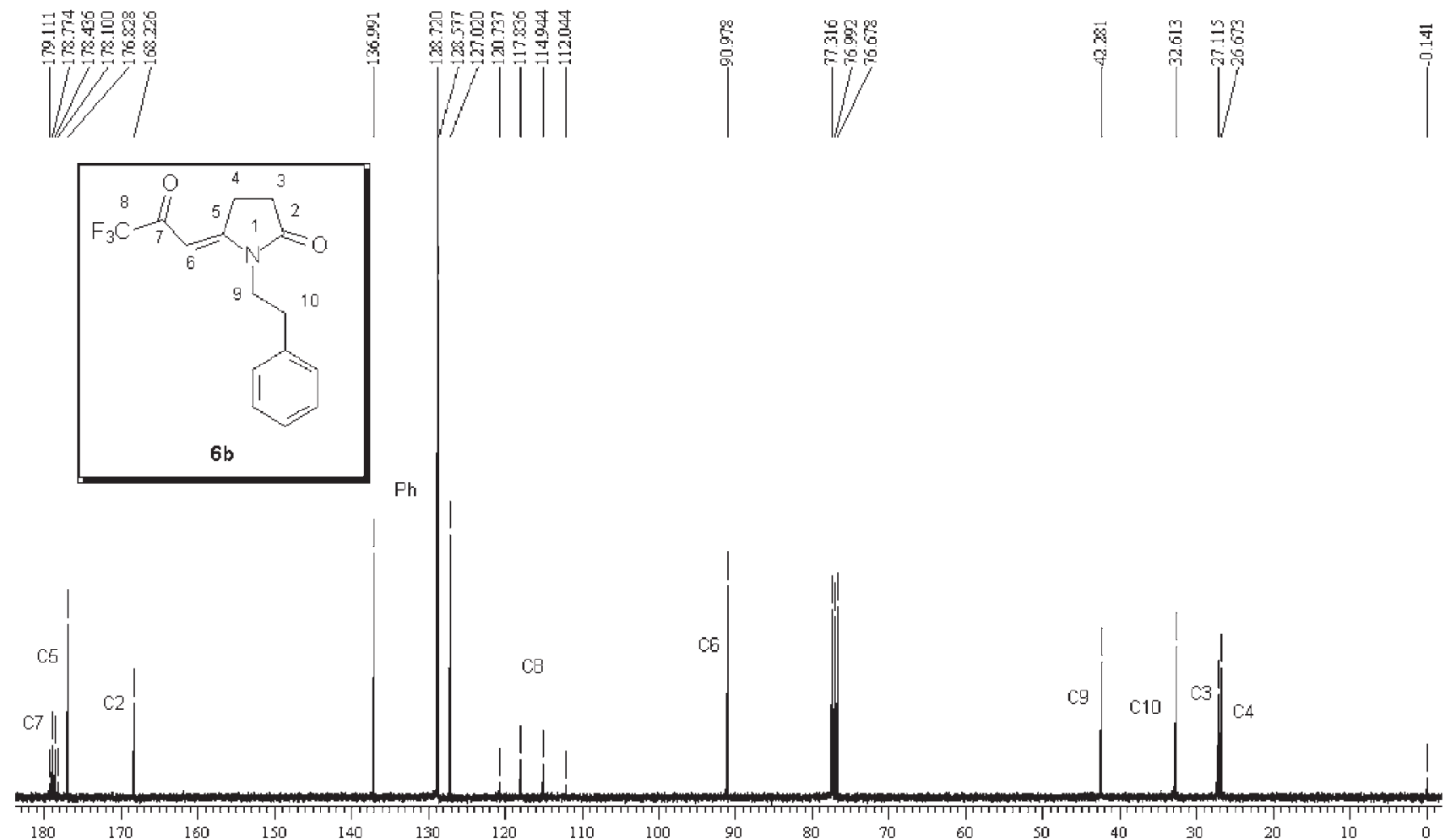

Figure S24. ${ }^{13} \mathrm{C}\{\mathrm{H}\}$ NMR spectrum from 1-(2-phenylethyl)-5-(3,3,3-trifluoropro-2-oxopylidene)pyrrolidin-2-one (5c), 100.62 $\mathrm{MHz}_{\text {in }} \mathrm{CDCl}_{3}$ using TMS 


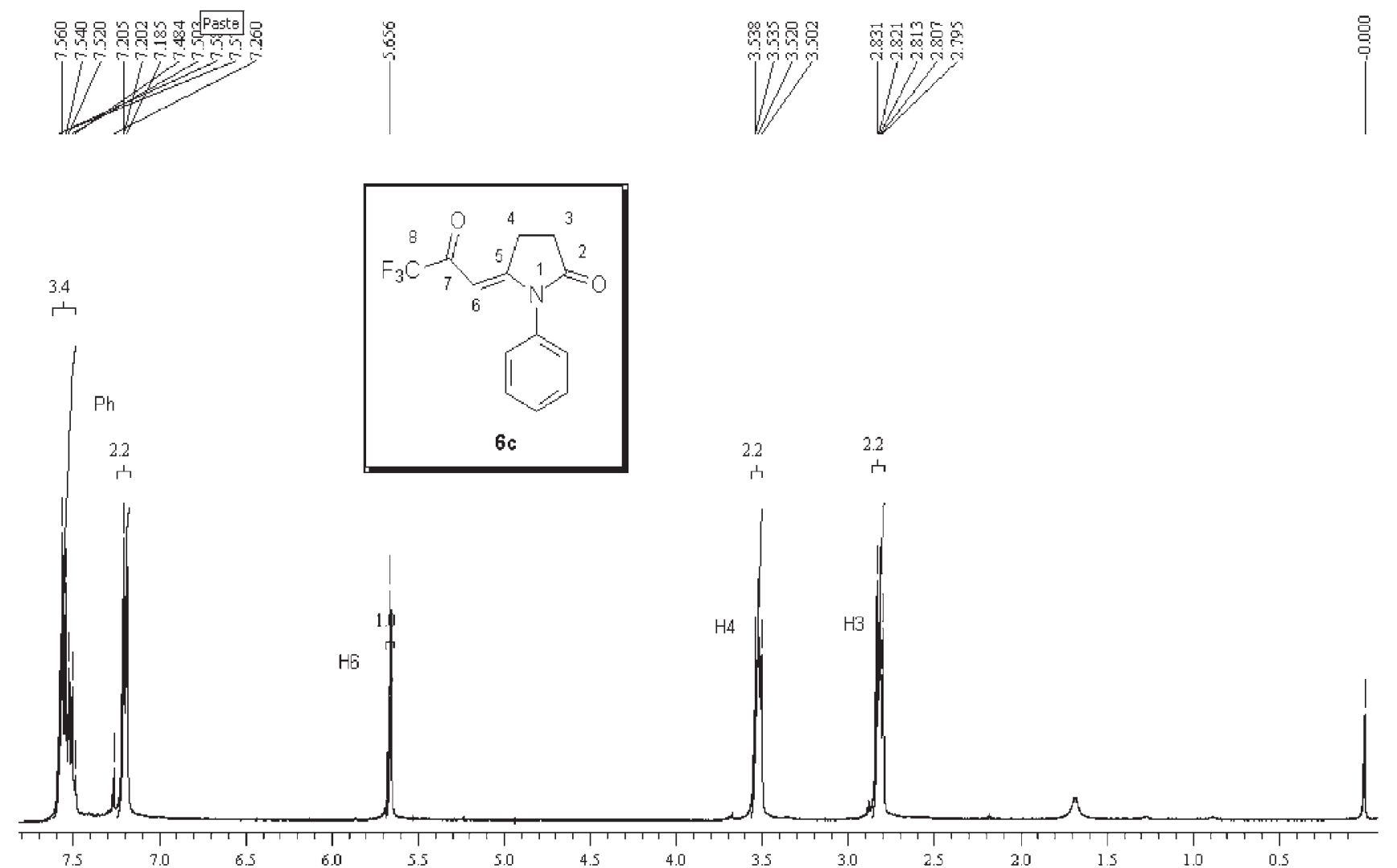

Figure S25. ${ }^{1} \mathrm{H}$ NMR spectrum from 1-phenyl-5-(3,3,3-trifluoro-2-oxopropylidene)pyrrolidin-2-one (5d), $400.13 \mathrm{MHz}$ in $\mathrm{CDCl}_{3}$ using TMS as internal standard.

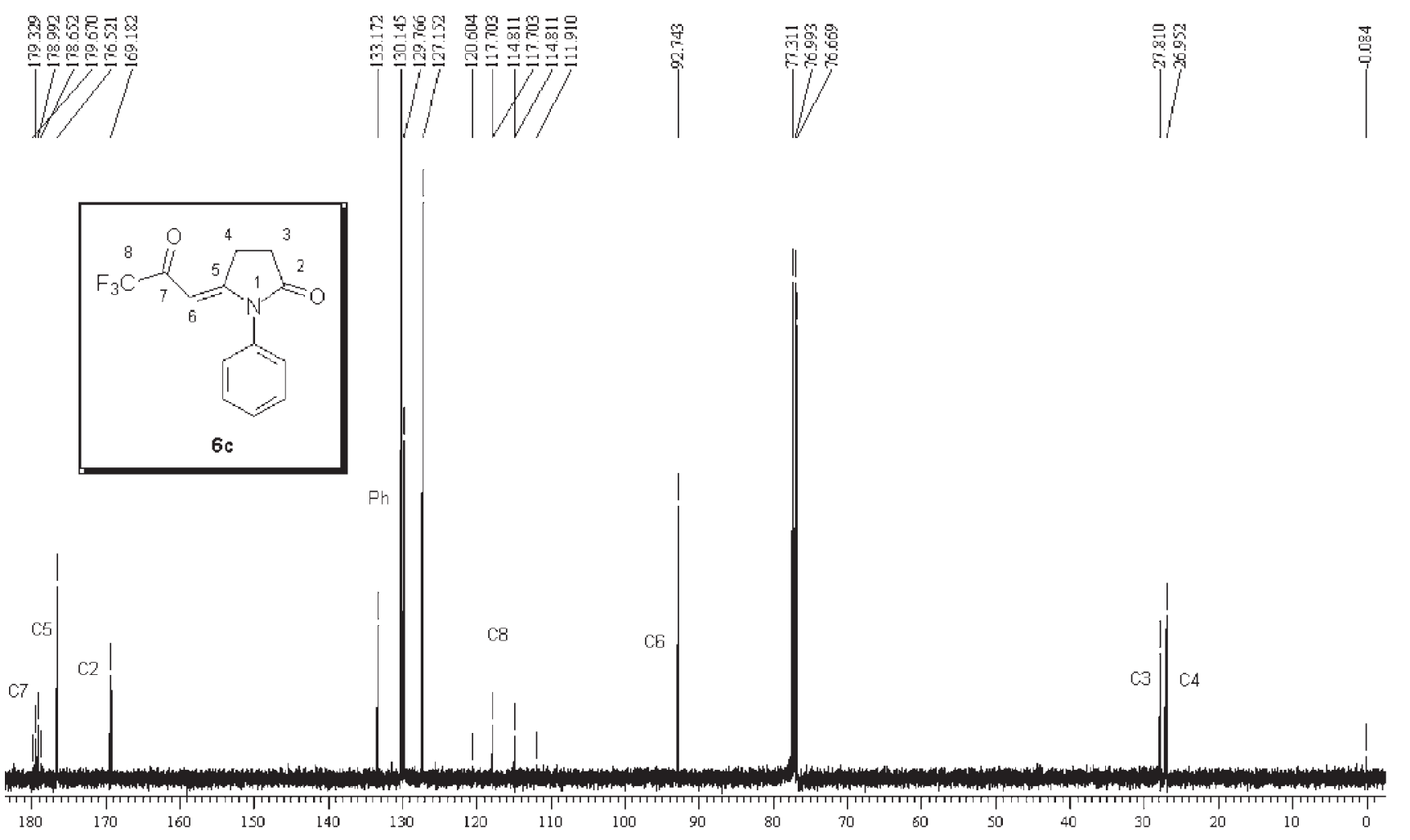

78 


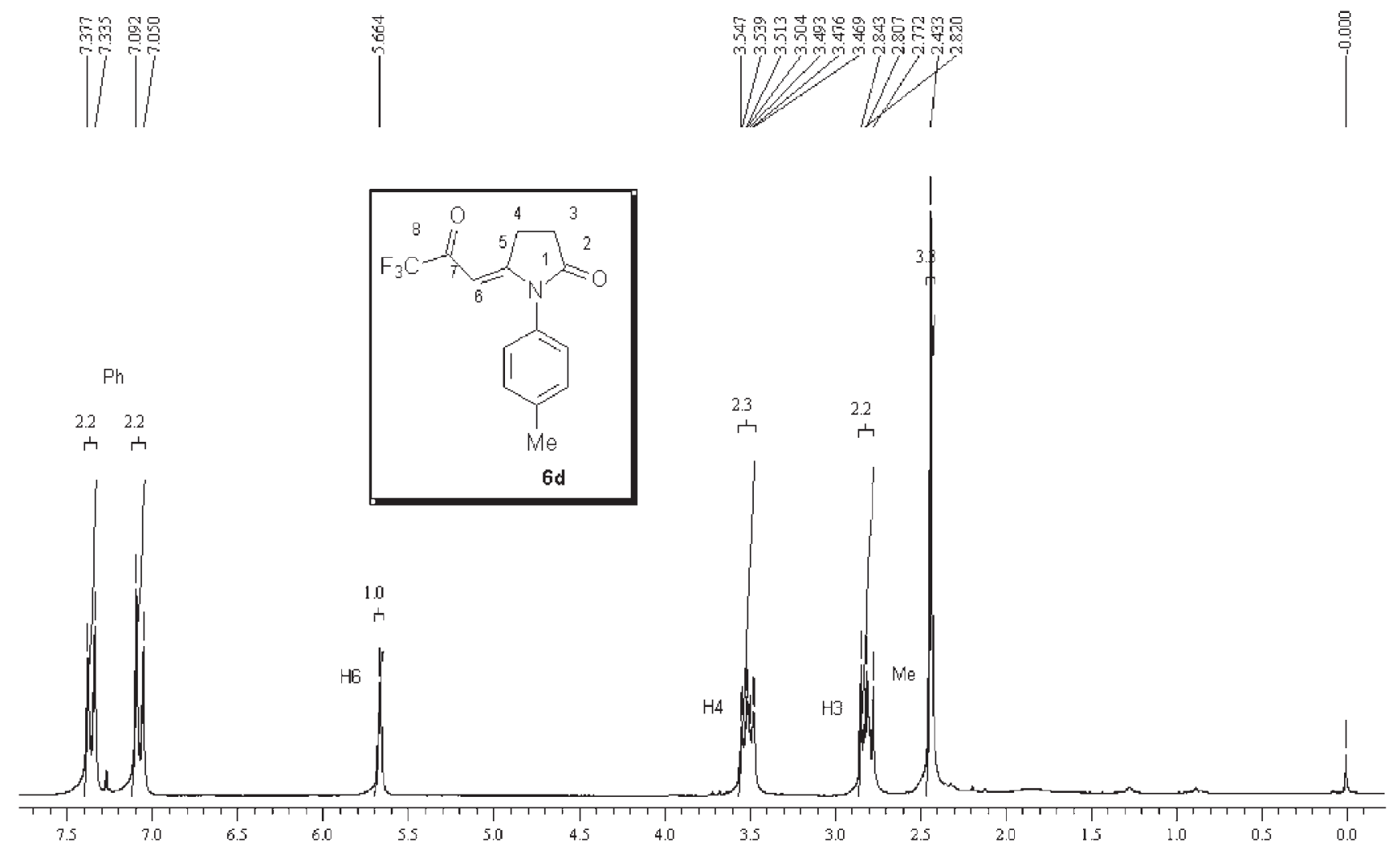

Figure S27. ${ }^{1} \mathrm{H}$ NMR spectrum from 1-(4-methylphenyl)- 5-(3,3,3-trifluoro-2-oxopropylidene)pyrrolidin-2-one (5e), $400.13 \mathrm{MHz}$ in $\mathrm{CDCl}_{3}$ using TMS

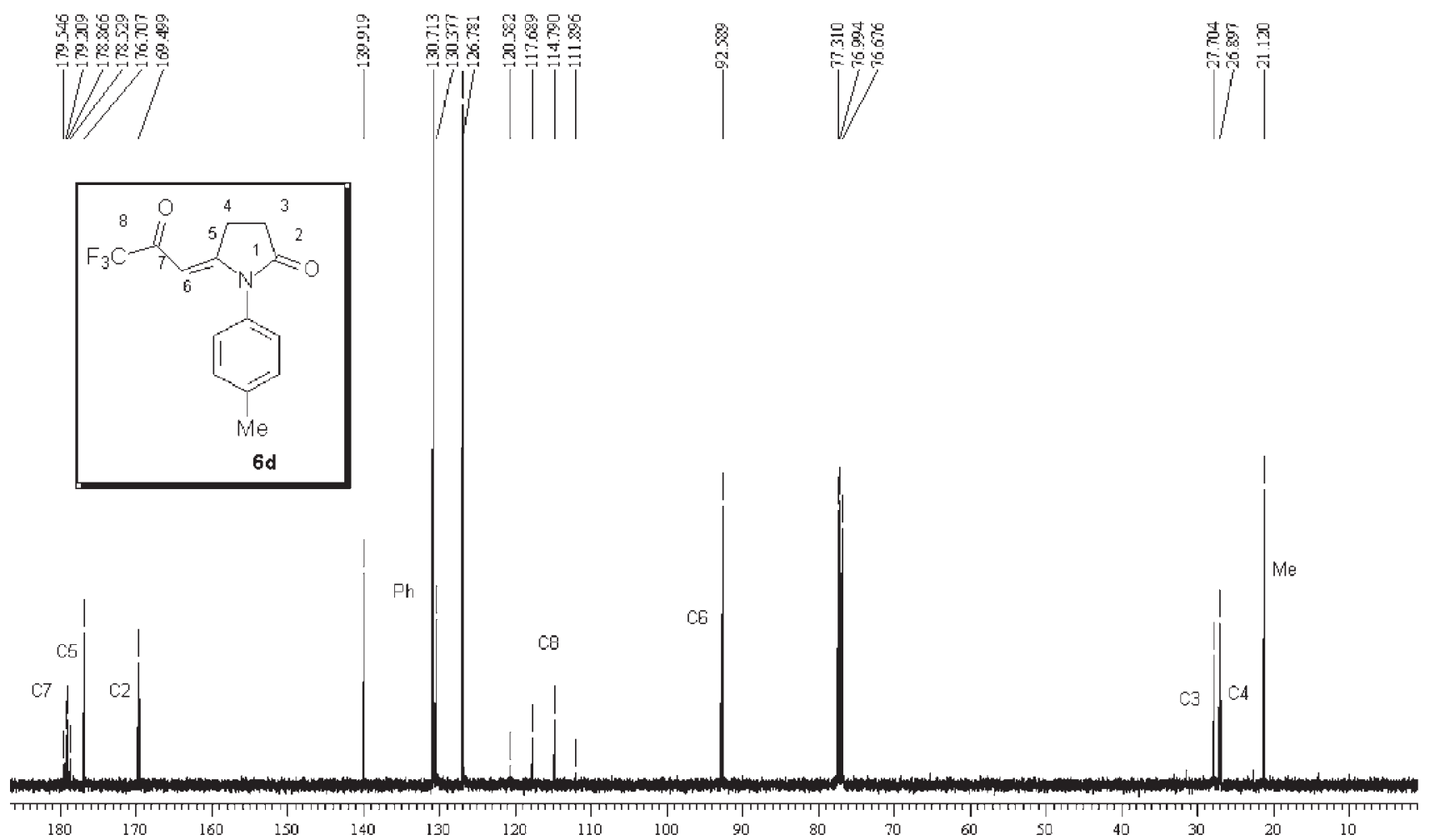

Figure S28. ${ }^{13} \mathrm{C}\{\mathrm{H}\}$ NMR spectrum from 1-(4-methylphenyl)- 5-(3,3,3-trifluoro-2-oxopropylidene)pyrrolidin-2-one (5e), $100.62 \mathrm{MHz}$ in $\mathrm{CDCl}_{3}$ using TMS as internal standard. 


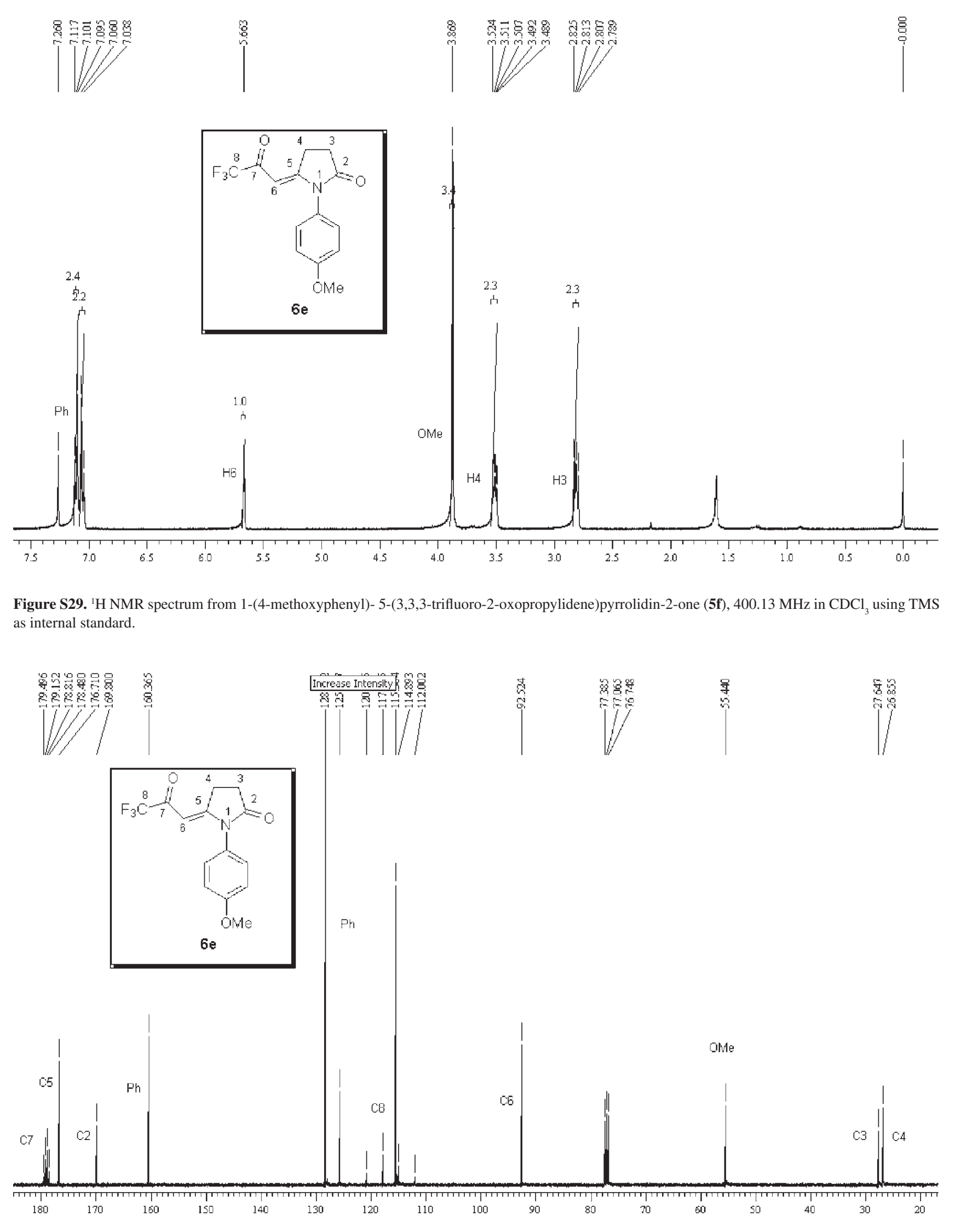

Figure S30. ${ }^{13} \mathrm{C}\{\mathrm{H}\}$ NMR spectrum from 1-(4-methoxyphenyl)-5-(3,3,3-trifluoro-2-oxopropylidene)pyrrolidin-2-one (5f), $100.62 \mathrm{MHz}$ in $\mathrm{CDCl}_{3}$ using TMS as internal standard. 
1
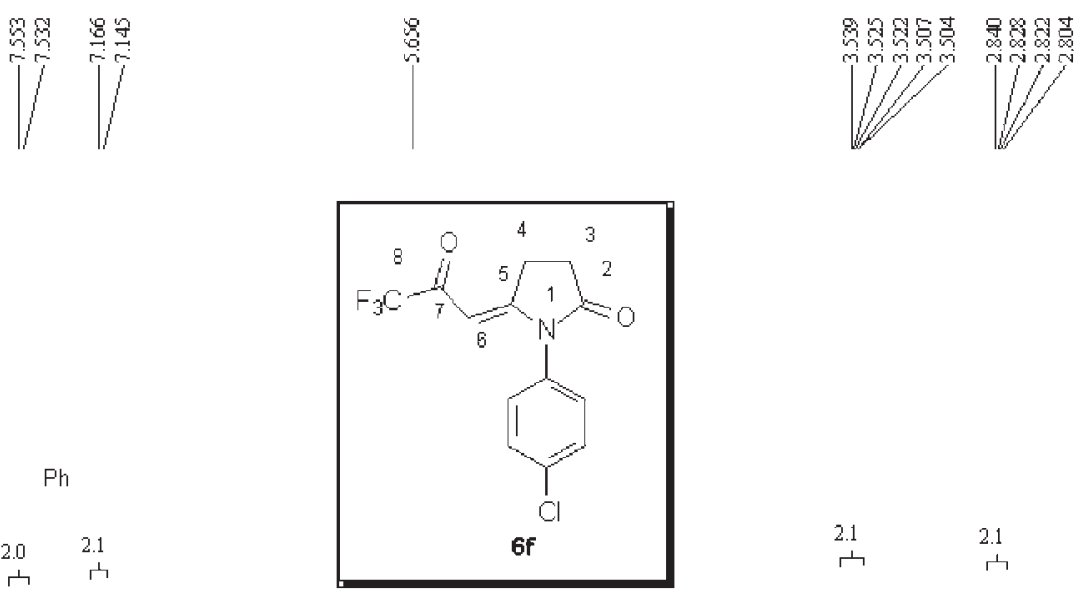

Ph
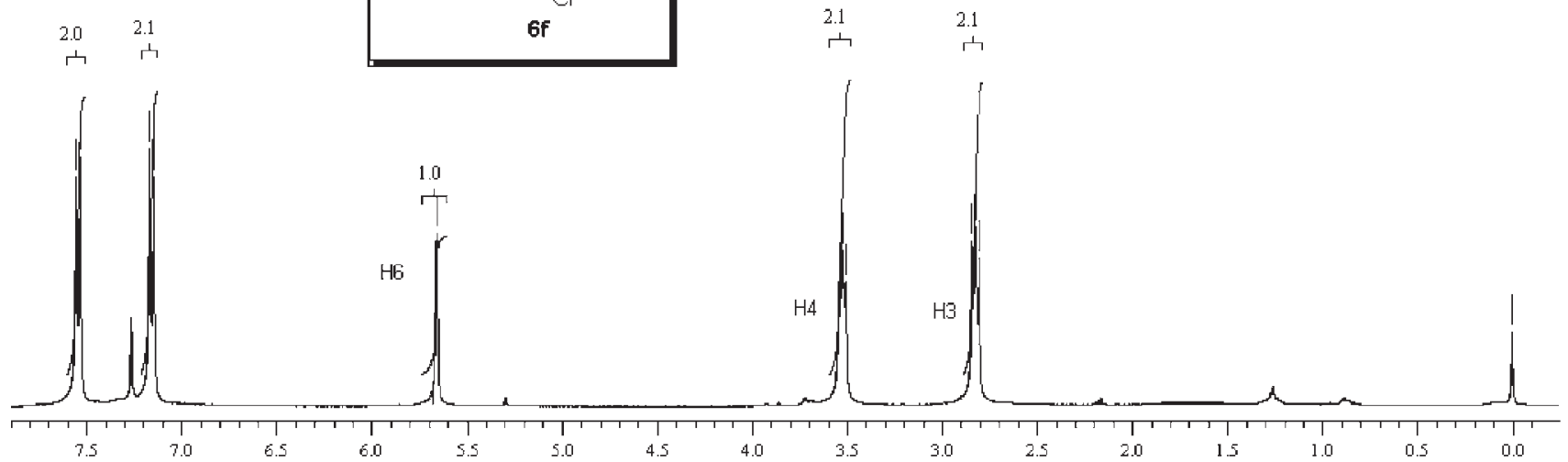

Figure S31. ${ }^{1} \mathrm{H}$ NMR spectrum from 1-(4-chlorophenyl)-5-(3,3,3-trifluoro-2-oxopropylidene)pyrrolidin-2-one (5g), $400.13 \mathrm{MHz}$ in $\mathrm{CDCl} 3$ using TMS

as internal standard.
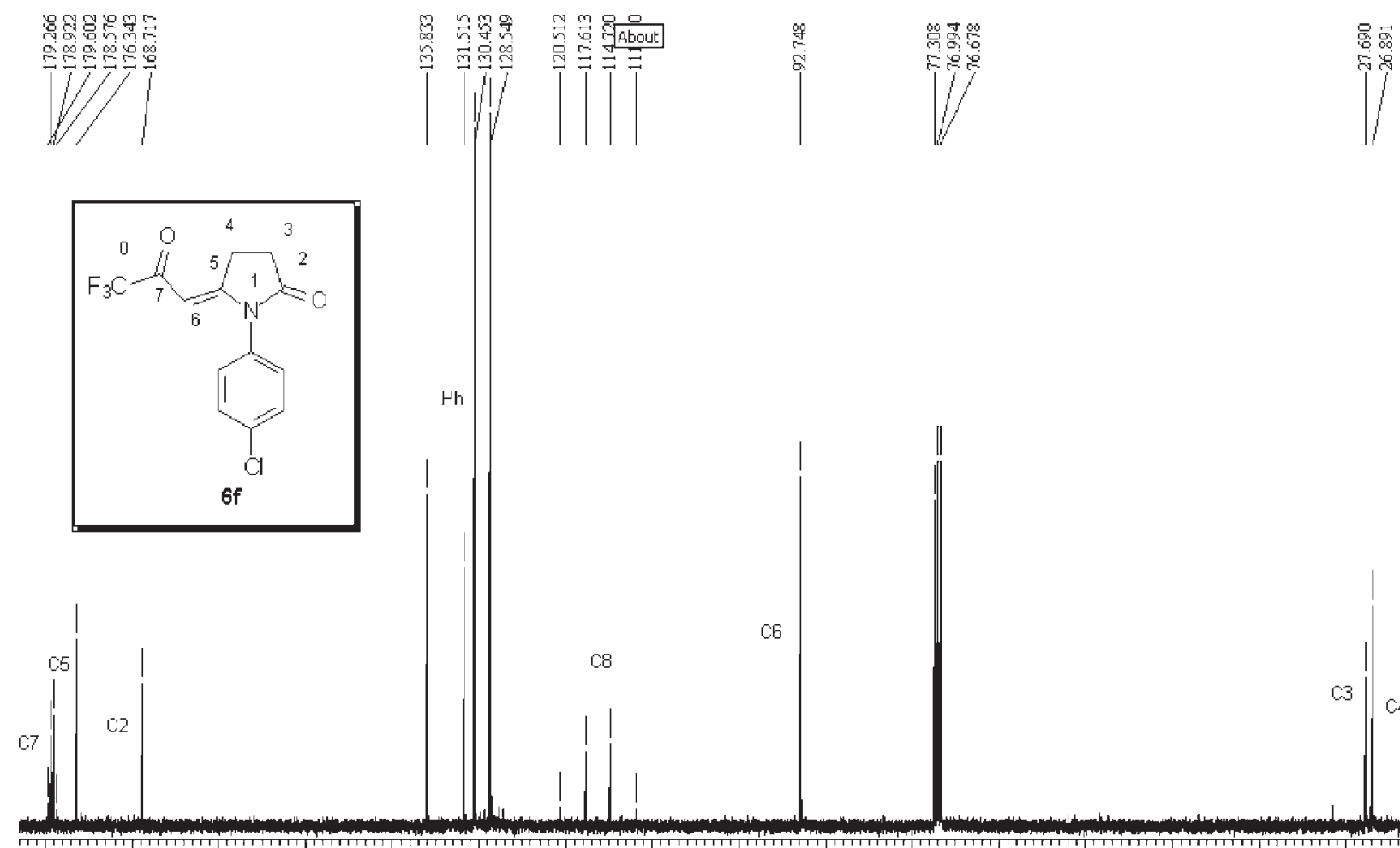

$\begin{array}{lllllllll}180 & 170 & 160 & 150 & 140 & 130 & 120 & 110 & 100\end{array}$

Figure S32. ${ }^{13} \mathrm{C}\{\mathrm{H}\}$ NMR spectrum from 1-(4-chlorophenyl)-5-(3,3,3-trifluoro-2-oxopropylidene)pyrrolidin-2-one (5g), 100.62 $\mathrm{MHz}$ in $\mathrm{CDCl}$, using TMS as internal standard. 


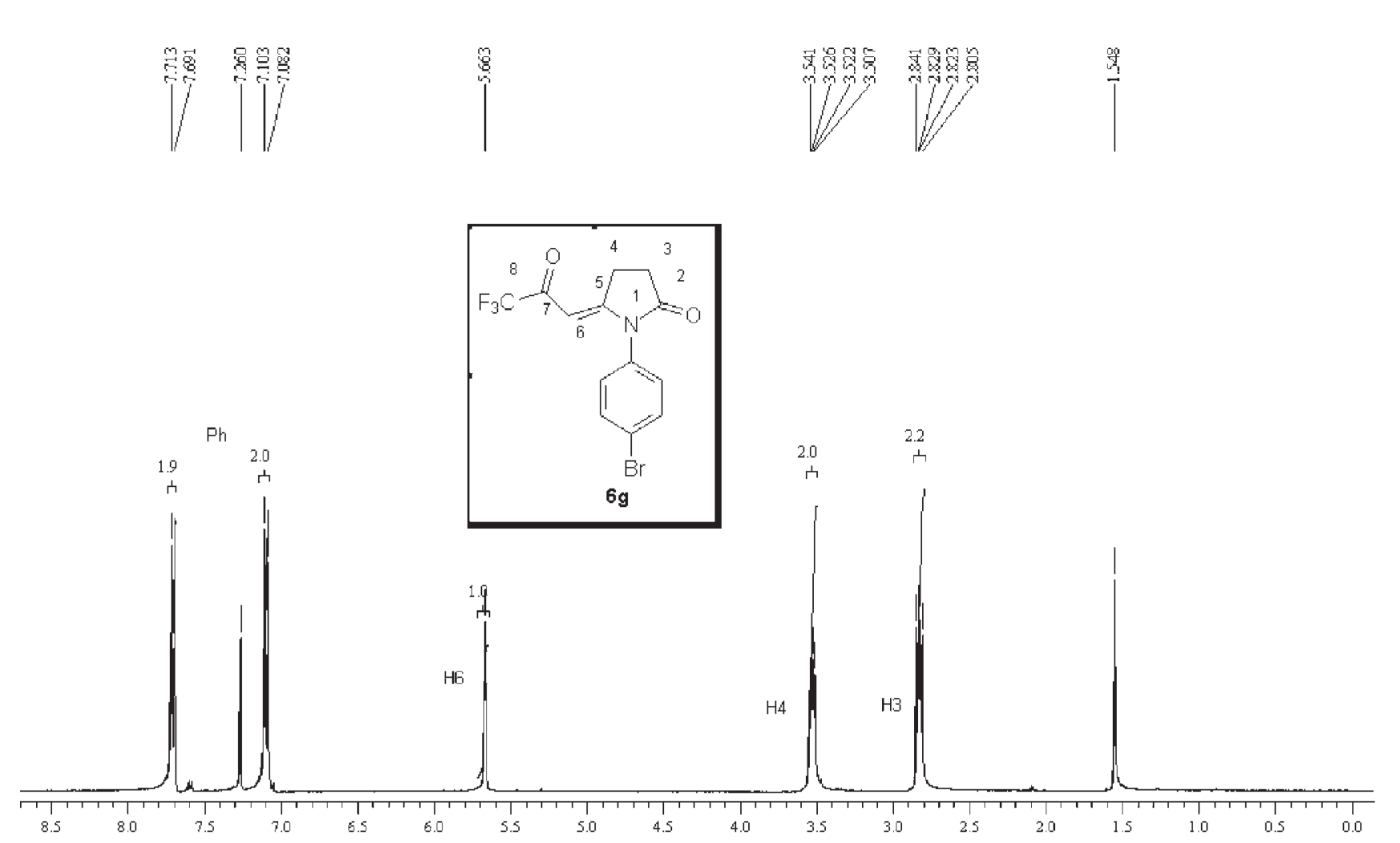

Figure S33. ${ }^{1} \mathrm{H}$ NMR spectrum from 1-(4-bromophenyl)-5-(3,3,3-trifluoro-2-oxopropylidene)pyrrolidin-2-one (5h), $400.13 \mathrm{MHz}$ in $\mathrm{CDCl}_{3}$ using $\mathrm{TMS}$ as internal standard.

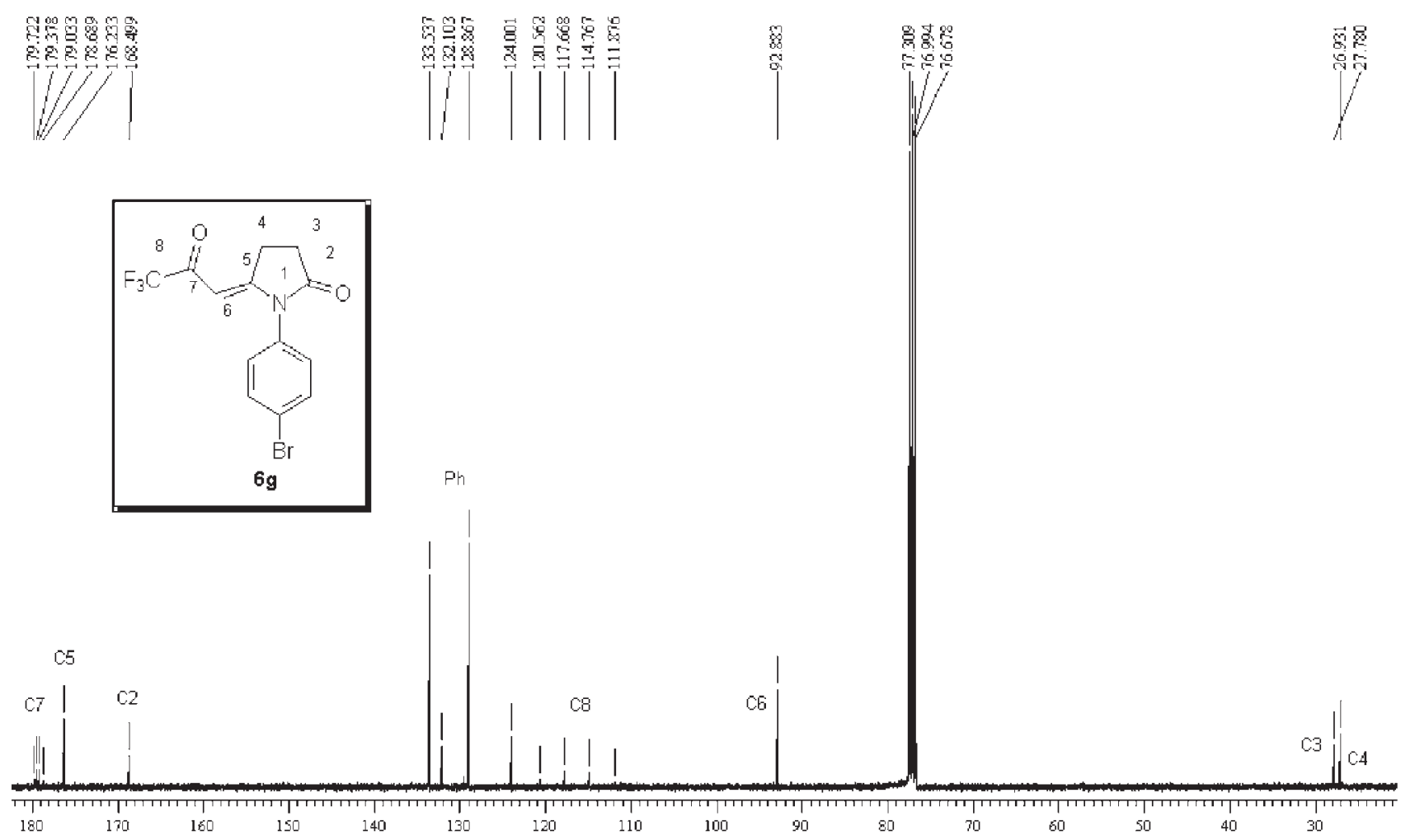

Figure S34. ${ }^{1} \mathrm{H}$ NMR spectrum from 1-(4-bromophenyl)-5-(,3,3,3-trifluoro-2-oxopropylidene)pyrrolidin-2-one (5h), $100.62 \mathrm{MHz}$ in $\mathrm{CDCl}_{3}$ using TMS as internal standard. 
1
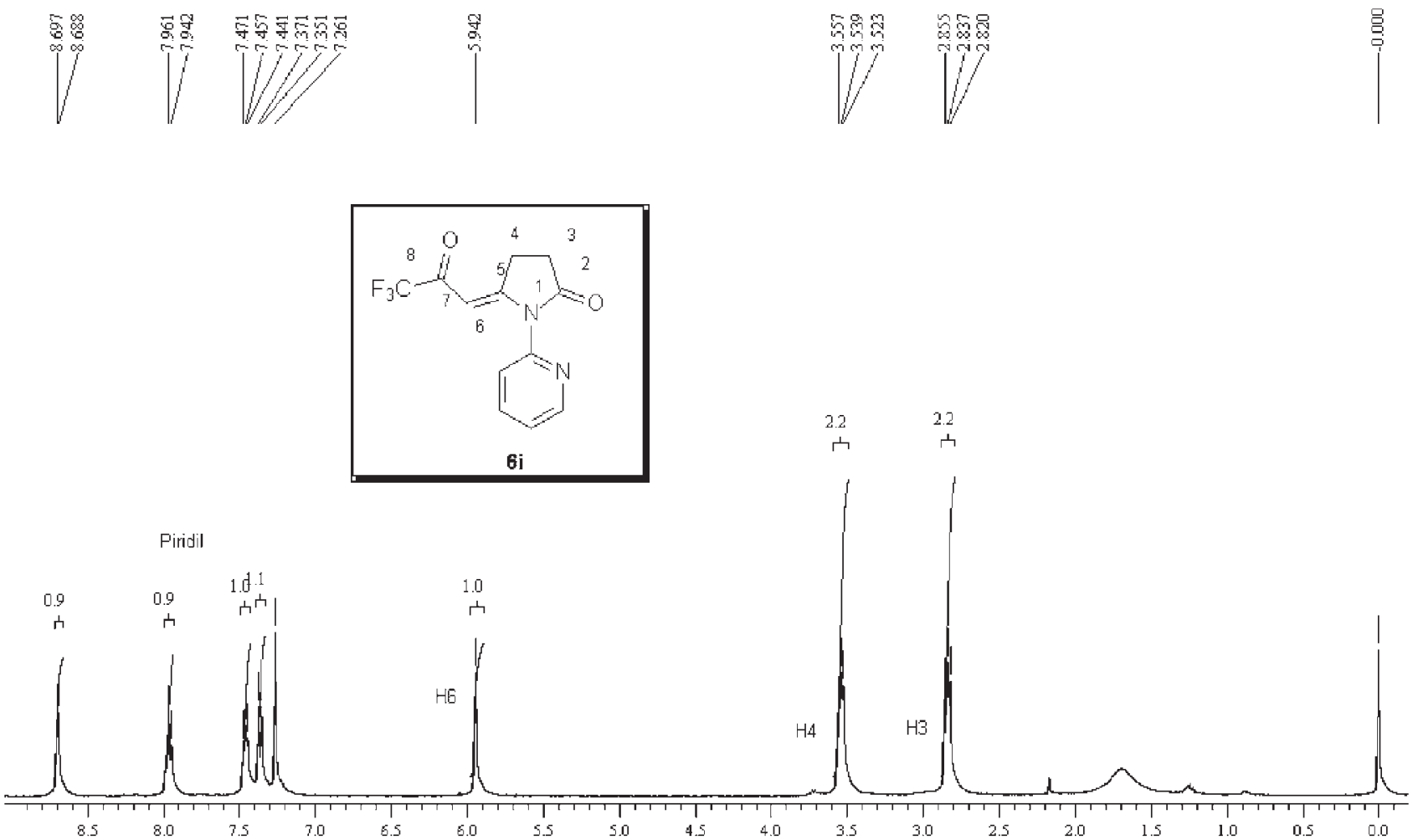

Figure S35. ${ }^{1} \mathrm{H}$ NMR spectrum from 1-(2-pyridyl)- 5-(3,3,3-trifluoro-2-oxopropylidene)pyrrolidin-2-one (5j), 400.13 $\mathrm{MHz}$ in $\mathrm{CDCl}$ 3 using TMS as

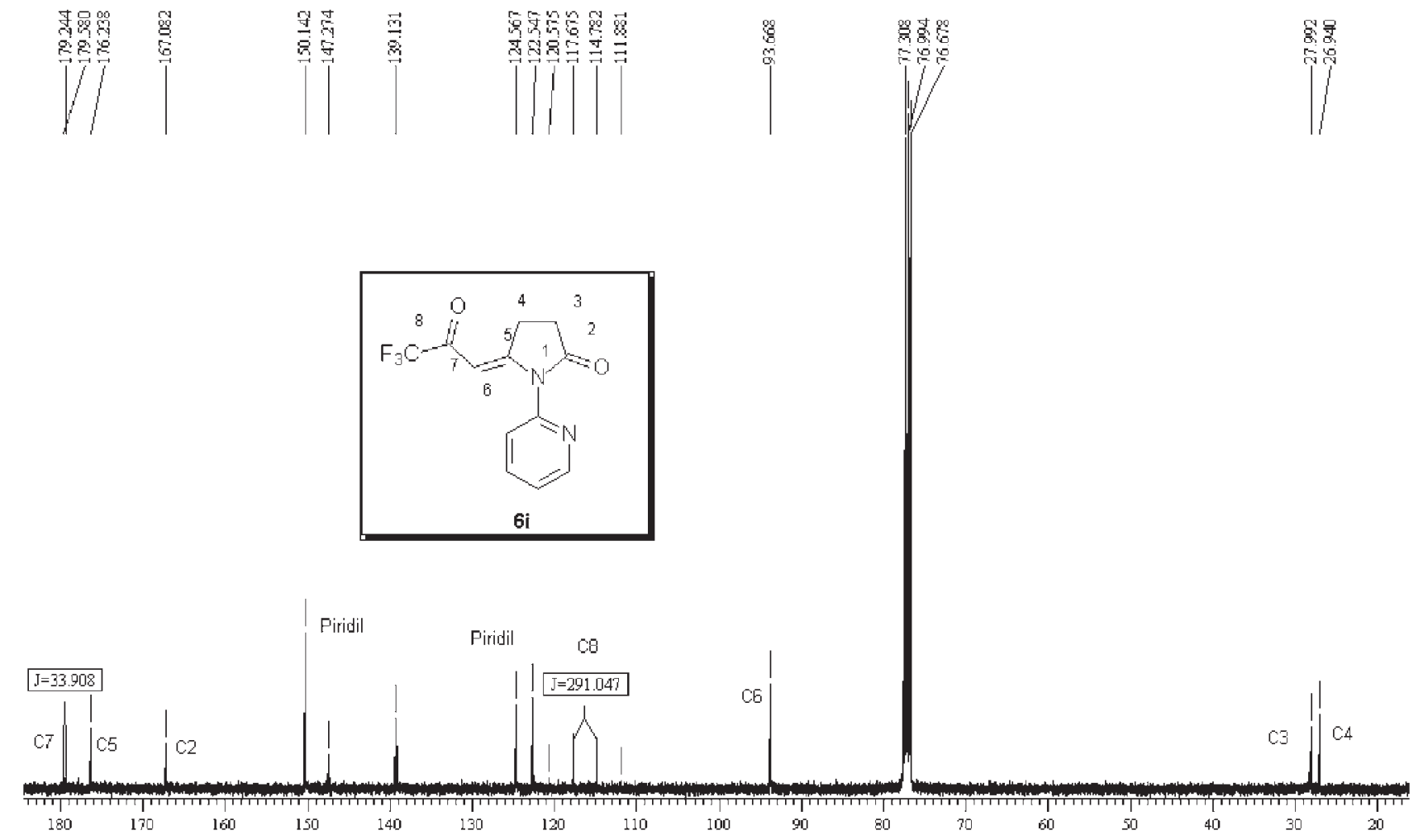

Figure S36. ${ }^{13} \mathrm{C}\{\mathrm{H}\}$ NMR spectrum from 2-oxo-1-(2-pyridyl)- 5-(3,3,3-trifluoro-2-oxo-propylidene)pyrrolidin-2-one (5j), 100.62 $\mathrm{MHz}$ in $\mathrm{CDCl}{ }_{3}$ using 


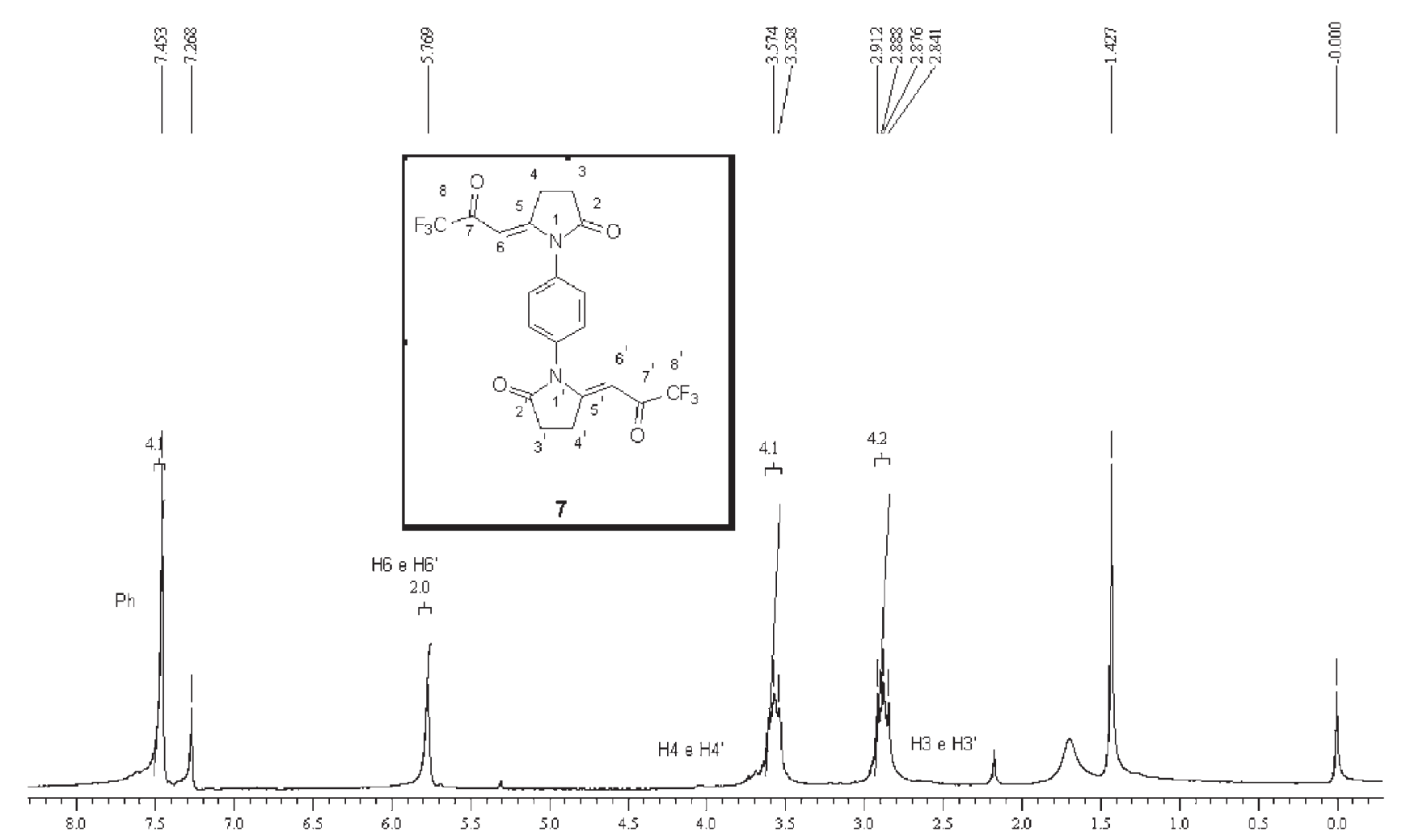

Figure S37. ${ }^{1} \mathrm{H}$ NMR spectrum from 1,4-(phenylene)bis(5-(3,3,3-trifluoro-2-oxopropylidene)pyrrolidin-2-one) (5k), $400.13 \mathrm{MHz}$ in $\mathrm{CDCl}_{3}$ using TMS as internal standard.
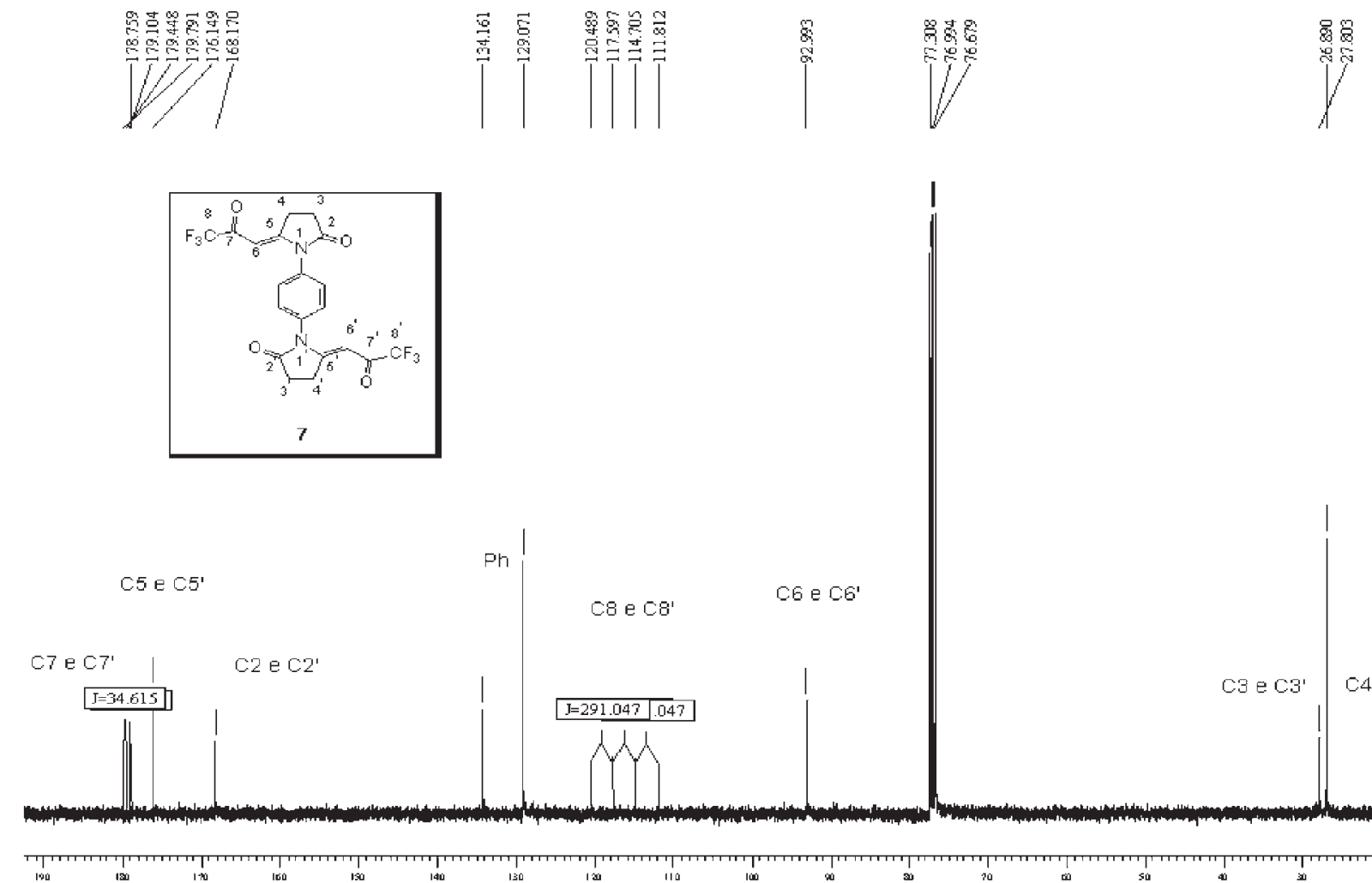

Figure S38. ${ }^{13} \mathrm{C}\{\mathrm{H}\}$ NMR spectrum from 1,4-(phenylene)bis(5-(3,3,3-trifluoro-2-oxopropylidene)pyrrolidin-2-one) (5k), 100.62 $\mathrm{MHz}$ in $\mathrm{CDCl}{ }_{3}$ using TMS as internal standard. 